\title{
A Cross-Layer Paradigm for Throughput and Energy Optimization in Distributed and Application-Centric Wireless Sensor Networks
}

Omar M. Sheikh, B.Eng., M.A.Sc.

A thesis submitted to the Faculty of Graduate and Postdoctoral Affairs

in partial fulfilment of the requirements for the degree of Doctor of Philosophy in Electrical and Computer Engineering

Ottawa-Carleton Institute for Electrical and Computer Engineering (OCIECE)

Department of Systems and Computer Engineering

Carleton University

Ottawa, Ontario, Canada, K1S 5B6

April 2011

(C) Copyright 2011, Omar M. Sheikh 
Library and Archives Canada

\section{Published Heritage} Branch

395 Wellington Street Ottawa ON K1A ON4 Canada
Bibliothèque et

Archives Canada

Direction du

Patrimoine de l'édition

395 , rue Wellington

Ottawa ON K1A 0N4

Canada
Your file Votre référence

ISBN: 978-0-494-81547-2

Our file Notre référence

ISBN: 978-0-494-81547-2
NOTICE:

The author has granted a nonexclusive license allowing Library and Archives Canada to reproduce, publish, archive, preserve, conserve, communicate to the public by telecommunication or on the Internet, loan, distribute and sell theses worldwide, for commercial or noncommercial purposes, in microform, paper, electronic and/or any other formats.

The author retains copyright ownership and moral rights in this thesis. Neither the thesis nor substantial extracts from it may be printed or otherwise reproduced without the author's permission.
AVIS:

L'auteur a accordé une licence non exclusive permettant à la Bibliothèque et Archives Canada de reproduire, publier, archiver, sauvegarder, conserver, transmettre au public par télécommunication ou par l'Internet, prêter, distribuer et vendre des thèses partout dans le monde, à des fins commerciales ou autres, sur support microforme, papier, électronique et/ou autres formats.

L'auteur conserve la propriété du droit d'auteur et des droits moraux qui protège cette thèse. $\mathrm{Ni}$ la thèse ni des extraits substantiels de celle-ci ne doivent être imprimés ou autrement reproduits sans son autorisation.
In compliance with the Canadian Privacy Act some supporting forms may have been removed from this thesis.

While these forms may be included in the document page count, their removal does not represent any loss of content from the thesis.
Conformément à la loi canadienne sur la protection de la vie privée, quelques formulaires secondaires ont été enlevés de cette thèse.

Bien que ces formulaires aient inclus dans la pagination, il n'y aura aucun contenu manquant. 
The undersigned recommend to the Faculty of Graduate and Postdoctoral Affairs acceptance of the thesis

A Cross-Layer Paradigm for Throughput and Energy Optimization in Distributed and Application-Centric Wireless Sensor Networks

submitted by

Omar M. Sheikh, B.Eng., M.A.Sc.

in partial fulfilment of the requirements for the degree of Doctor of Philosophy in Electrical and Computer Engineering

Chair, Howard Schwartz, Department of Systems and Computer Enginecring

Thesis Supervisor, Samy A. Mahmoud

External Examiner, Xavier Fernando, Faculty of Engineering, Architecture and Science, Ryerson University

Carleton University

April 2011 


\section{Abstract}

This thesis presents a cross-layer policy for joint throughput and energy optimization in next-generation wireless sensor networks (WSNs). We derive the link suitability of a transmission channel, which determines the optimal physical (PHY) layer resource allocation that jointly maximizes achievable throughput and minimizes the associated power cost of using the link. This differs from current approaches that fit into one of two categories: those that perform rate control but do not model power cost in terms of the achievable throughput on the transmission link, or those that do not separate performance-related benefits and energy-related costs to ensure that we can jointly maximize throughput and minimize energy consumption. Our method ensures that WSNs allocate transmission resources that impact limited sensor energies to operating channels capable of a higher level of throughput and energy conservation.

Using link suitability in a cross-layer policy, we contribute a cross-layer optimization protocol that is unique in identifying optimal routing paths with the highest trade-off between throughput benefits and energy costs across multiple transmission channels. Our policy is based on transmit power at the PHY layer, channel allocation at the link layer, and multi-hop path selection at the network layer. 
A Network Planning Protocol (NPP) works in conjunction with our cross-layer policy and contributes the relationship between: expected throughput performance, network lifetime, message load for data and coordination packets, and energy capacity. This relationship enables network planners of next-generation WSNs to study the impact of various network design decisions on performance and network lifetime metrics. This is achieved by deriving NPP in terms of physical resources that affect throughput and energy capacity such that we may allocate resources appropriately to meet network objectives.

We present these contributions for distributed and application-centric WSNs. Application-centric WSNs refer to sensor networks that execute applications with significant performance requirements, while incorporating energy conservation schemes for sensors. In many instances, it shall also be necessary to execute many of these sensory applications simultaneously, with centralized control of geographically distributed sensor clusters. As a result, this thesis explores the use of overlay mesh networks to design a two-ticr, applicatıon-centruc and distributed WSN archttecture. 


\section{Acknowledgments}

First and foremost, I would like to acknowledge my supervisor, Prof. Samy A. Mahmoud. His guidance and knowlcdge have been instrumental in this work.

Secondly, I would like to thank my parents, Dr. Munir and Yasmin Shcikh, for their encouragement, support and understanding throughout the last four years. The hard work and passion that I have put into this dissertation is a reflection of how you raised me. To my brothers, Osmaan and Hasan, thanks for always pushing me to do more. To my cousins Farah, Khadeeja, Kamran, Sadia, Jawad and others, thank you for the support.

Thirdly, I would like to acknowledge the contributions of Mohamed Abou El Saoud and Osama Aboul-Magd whose insightful discussions helped make this work possible.

Last but not least, to my friends, I thank you for all the moments in the last four years that have contributed to this achievement.

This dissertation is dedicated to the memory of my neighbor and friend, Omar. 


\section{Table of Contents}

Abstract $\quad$ iii

Acknowledgments $\quad$ v

List of Tables $\quad$ xii

List of Figures xiii

List of Acronyms $\quad$ xvi

1 Introduction $\quad 1$

1.1 Wireless Mesh Networks ................... 4

1.1.1 Wireless Sensor Networks .............. 6

1.2 State-of-the-Art in Mesh Networks . . . . . . . . . . . . 7

1.2.1 Communication Technologics for Mesh Networks . . . . . . . 8

1.2.2 High-Speed Mesh Networks . . . . . . . . . . . . 13

1.3 Motivation . . . . . . . . . . . . . . . 17

1.3.1 The Throughput Problem . . . . . . . . . . . . . . 17 
1.3.2 Optimization in Software Radio Systems . . . . . . . . . 18

1.3.3 Overlay Mesh Networks as an Enabling Technology . . . . . 20

1.3.4 Cross-Layer Design in WSNs . . . . . . . . . . . . . . 22

1.4 Problem Statement . . . . . . . . . . . . . . . . . 24

1.5 Contributions of this Research . . . . . . . . . . . . . 26

1.6 Outline of Thesis . . . . . . . . . . . . . . . . 27

$\begin{array}{llr}2 & \text { Background } & 29\end{array}$

2.1 Introduction . . . . . . . . . . . . . . . . . . . . . . . 29

2.2 Literature Review . . . . . . . . . . . . . . . . . 31

2.2.1 Link Suitability and Throughput Modeling . . . . . . . . . 32

2.2.2 Cross-Layer Optimization in WSNs . . . . . . . . . . . . 37

2.2 .3 Network Planning . . . . . . . . . . . . . . . . . . 38

2.2 .4 Routing in WSNs . . . . . . . . . . . . . 40

2.2 .5 Clustering . . . . . . . . . . . . . . . . . 49

2.2.6 Preference Modeling and Utility Functions . . . . . . . . . 56

2.3 PHY Layer Resources and FDMA Capacity . . . . . . . . . . . . . 61

2.4 Modeling Energy Efficiency . . . . . . . . . . . . . 62

2.4 .1 Power Consumption due to a Transmission . . . . . . . . . . 63

2.5 Critique of Literature . . . . . . . . . . . . . . 65

2.6 Summary . . . . . . . . . . . . . . . . 66

$\begin{array}{llr}3 & \text { Problem Formulation } & 68\end{array}$ 
3.1 Introduction . . . . . . . . . . . . . . . 68

3.2 Network Architecture . . . . . . . . . . . . . . . . 70

3.3 Problem Definition . . . . . . . . . . . . . . . . 72

3.4 Optimization Parameters of Interest . . . . . . . . . . . . . 74

3.5 Assumptions . . . . . . . . . . . . . . . . . . . . 75

3.6 Link Utility Function . . . . . . . . . . . . . . . . . 78

3.7 Objective Function $\ldots \ldots \ldots \ldots$. . . . . . . . . 86

3.7.1 Optimized Link Utility . . . . . . . . . . . . 87

3.7 .2 Constraints . . . . . . . . . . . . . . . . 89

3.8 Radio Models for Wireless Technologies . . . . . . . . . . . . . . . 91

3.8 .1 Path Loss . . . . . . . . . . . . . . . . . . 92

3.9 Summary . . . . . . . . . . . . . . . . . . 93

4 Cross-Layer Design $\quad 95$

4.1 Introduction $\ldots \ldots \ldots \ldots \ldots \ldots$

4.2 Protocol Stack Design _. . . . . . . . . . . . . . . . . 96

4.3 Node States . . . . . . . . . . . . . . . . . . . . . . . . 98

4.4 Network Layer . . . . . . . . . . . . . . . . . . . . . . 99

4.4 .1 Initialization State . . . . . . . . . . . 100

4.4 .2 Path Optimization State . . . . . . . . . . . 111

4.5 Data Link Layer . . . . . . . . . . . . . . . . . . . 113

4.5.1 Channel Optimization State . . . . . . . . . . . 113 
4.6 PHY Layer . . . . . . . . . . . . . . . . . . . . . . 116

4.6.1 Link Optimization State . . . . . . . . . . . . . 116

4.7 Steps in Cross-Layer Optimization Process . . . . . . . . . . . . . . . 117

4.8 Summary . . . . . . . . . . . . . . . . . . . . . . . . . . 119

5 System Design $\quad 120$

5.1 Mesh Network Deployment . . . . . . . . . . . . . . . . . . . . 121

5.2 Node State Information . . . . . . . . . . . . . . . . . . . . 122

5.3 Message Formats . . . . . . . . . . . . . . . . . . . . . . . . 123

5.3.1 Hello Messages for Presence Broadcast . . . . . . . . . . . . . 123

5.3 .2 Routing Decision Updates . . . . . . . . . . . . . . . 125

5.3.3 Data Packets . . . . . . . . . . . . . . . . 128

5.4 Network Planning for Predictable Lifetimes . . . . . . . . . . . . 128

5.4 .1 Sensors . . . . . . . . . . . . . . . . . 130

5.4 .2 Cluster-Heads . . . . . . . . . . . . . . . . . 137

5.4 .3 Mesh Relay Nodes . . . . . . . . . . . . . . . . . . . . . 141

5.5 Queueing Model . . . . . . . . . . . . . . . . . . . . 143

5.6 Simulation Methodology . . . . . . . . . . . . . . . . . . 146

5.6 .1 Optimization Variables . . . . . . . . . . . . . 146

5.7 Initial Energy Capacity Modeling . . . . . . . . . . . . . . . . . . . 148

5.8 Evaluative Metrics . . . . . . . . . . . . . . . . . . 150

5.8 .1 Throughput . . . . . . . . . . . . . . . . 151 
5.8.2 Percentage of Remaining Energy Capacities . . . . . . . . . 151

5.8.3 Mean Number of Network Connections . . . . . . . . . . . . . 152

5.8.4 Blocking Probability . . . . . . . . . . . . . . 152

5.8.5 Expected Waiting Times with High Priority Clusters . . . . . 154

5.9 Summary . . . . . . . . . . . . . . . . . . 158

6 Performance Evaluation $\quad 160$

6.1 Introduction . . . . . . . . . . . . . 160

6.2 Convergence and Nelder-Mead Method for Nonlinear Optimization . . 161

6.3 Simulation Results . . . . . . . . . . . . . . . . . . 162

6.3 .1 Total Network Throughput . . . . . . . . . . . . 163

6.3.2 Mean Per-Hop Throughput . . . . . . . . . . . . 165

6.3 .3 Spectral Efficiency . . . . . . . . . . . . . 166

6.3.4 Average Number of Hops per Path . . . . . . . . . . . 167

6.3.5 Percentage of Remaining Energy Capacities . . . . . . . . . . 169

6.3.6 Mean Number of Network Connections . . . . . . . . . . . 171

6.3.7 Blocking Probability . . . . . . . . . . . . . 172

6.3.8 Expected Waiting Times with High Priority Clusters . . . . . 178

6.4 Summary . . . . . . . . . . . . . . . . . . 180

$\begin{array}{llr}7 & \text { Conclusion } & 183\end{array}$

7.1 Practical Simplifications to Reduce Time Complexity . . . . . . . . . 187

7.2 A Discussion on Software and Cognitive Radio . . . . . . . . . . 188 
7.3 Contributions . . . . . . . . . . . . . . . . . 190

7.4 Future Work . . . . . . . . . . . . . . . . . . . . 191

7.4.1 Modulation, Bandwidth and Application Factors . . . . . . . . 191

7.4 .2 Sensor Localization . . . . . . . . . . . . . . . . . . 192

7.4.3 Application Queries and Addressing . . . . . . . . . . . 193

7.4.4 Back-Up Mesh Node Scheduling . . . . . . . . . . . . . 193

$\begin{array}{ll}\text { List of References } & 195\end{array}$

Appendix A Summary of the Cross-Layer Optimization Policy for $\begin{array}{ll}\text { Path, Channel, Power and Rate Negotiation } & 209\end{array}$

Appendix B Link Budget Analysis for Ultrawideband (UWB), Zigbee $\begin{array}{ll}\text { and WiMax } & \mathbf{2 1 1}\end{array}$

Appendix C Proof of Convergence for $L_{v_{x}, v_{y}, f}^{*}$ and $U_{v_{z}, v_{j}}^{a^{*}}$ 


\section{List of Tables}

1.1 Typical Data Rates and Frequency of Operation for Different Standards 10

1.2 Energy Efficiency Coefficients for Various Standards . . . . . . . . . 11

3.1 Comparison of Operating Parameters for Zigbee, UWB and WiMax

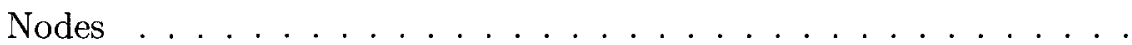

4.1 Optimized Link Utilities $L^{*}$ for UWB Channels for Candidate Simple

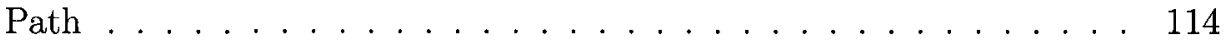

4.2 Preferred Channels in Order of Optimized Link Utility $L^{*} \ldots \ldots$

4.3 Preferred Channels Where First Preference is Not Valid . . . . . . . 115

5.1 Selection of Optimization Parameters in the Heterogeneous WSN . . 147

5.2 Communication Parameters for Three Sub-Networks . . . . . . . . . 148

5.3 Required and Applied Energy Capacities for Sensors, Cluster-Heads and Mesh Relay Nodes for One Week Network Lifetime . . . . . . . 150

6.1 Throughput Statistics _. . . . . . . . . . . . . . 163

B.1 A Link Budget Analysis for Zigbee, UWB and WiMax . . . . . . . 211

C.1 Summary of $\Delta\left(P_{v_{x}, f}^{t}, t\right)$ for Zigbee, UWB and WiMax Networks . . . 213 


\section{List of Figures}

1.1 A Sensor Network with Multi-Hop Paths . . . . . . . . . . . . 7

1.2 A Two-Tier Multi-Hop Sensor Network Architecture . . . . . . . . . . 22

1.3 Various Cross-Layer Design Protocols . . . . . . . . . . . . . . . . 23

1.4 CLASS, a proposed cross-layer stack for wireless systems . . . . . . . 24

2.1 The Flow Augmentation Algorithm . . . . . . . . . . . . . 47

2.2 A Comparison of Absolute and Relative Residual Energies . . . . . 48

2.3 Consideration of Flow Consumption Energy . . . . . . . . . . . . 49

2.4 Field Life for the Exemplary and Reconfigured Sensor Networks . . . 55

3.1 The Distributed WSN Architecture . . . . . . . . . . . . . 71

3.2 Example of a Link Utility Function $L_{v_{x}, v_{y}, f}\left(R_{v_{x}, v_{y}, f}, t\right) \ldots \ldots$

3.3 Examples of Benefit and Cost Functions to Form Link Utility . . . . 81

4.1 Protocol Stack Model for Application-Centric WSNs . . . . . . . . 97

4.2 New Path Advertisements in Simple Path Protocol (SPP) . . . . . . . 101

5.1 Back-up Cluster-Heads and Master Nodes in Distributed WSN . . . . 121

5.2 Hello Packet . . . . . . . . . . . . . . . . . . . . . . . . . . . . . . . . 124

5.3 Propagation of RDU through Sensor and Mesh Networks . . . . . . 126 
5.4 routing decision update (RDU) Payload . . . . . . . . . . . . 127

5.5 Duration Node Remains Awake Upon Receiving RDU, $T_{A W_{R D U}}+T_{A W_{\text {data }}} 133$

5.6 Relay Nodes in a Sample Path in the Sensor Cluster . . . . . . . . . . 134

5.7 Energy Capacities vs. Desired Network Lifetime for Various UWB Sensor Message Loads . . . . . . . . . . . . . . . . . . . . . 135

5.8 State Transition Diagram For M/M/c/c Queueing System . . . . . 144

5.9 Energy Savings by UWB over One Week Network Lifetime . . . . . . 149

6.1 Mean Per-Hop Throughput over One Week Network Lifetime . . . . . 165

6.2 Spectral Efficiency (bits/s/Hz) over One Week Network Lifetime . . . 166

6.3 Average Number of Hops Per Path in Each Sub-Network . . . . . . . 168

6.4 Comparison of Remaining Battery Energies Over One Week Network Lifetime . . . . . . . . . . . . . . . . . . . 169

6.5 Remaining Network Energies in Last 12 Hours with First Node Outages 170

6.6 Mean Network Connections In Last Six Hours . . . . . . . . . . . . . 172

6.7 Network Blocking Probability vs. Traffic Intensity, $\rho \ldots \ldots$

6.8 Network Blocking Probability vs. Number of Operating Channels, F 174

6.9 Blocking Probability of UWB and Zigbee Clusters vs. Traffic Intensity,

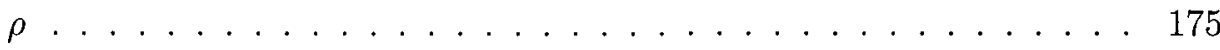

6.10 Blocking Probability of UWB and Zigbee Clusters vs. Number of Operating Channels $F \ldots \ldots \ldots \ldots$. . . . . . . . . . . . . . .

6.11 Percentage of Available Channels for $F=10$ and $\rho=1$ In Three Subnetworks . . . . . . . . . . . . . . . . . 177 
6.12 Expected Waiting Time for High and Low Priority Requests with $p_{h}$ $=25 \%$ and $\mu=10$ requests $/$ sec $\ldots \ldots \ldots \ldots \ldots$

A.1 Summary of the Cross-Layer Optimization Policy for Path, Channel, Power and Rate Negotiation . . . . . . . . . . . . . . . . 210 


\title{
List of Acronyms
}

\author{
ANDA Ad Hoc Network Design Algorithm \\ AWGN additive white Gaussian noise \\ BER bit error rate \\ BS base station \\ CPU central processing unit \\ DSC Distributed Source Coding \\ DSSS direct sequence spread spectrum \\ EAR Energy Aware Routing \\ EIRP Effective Isotropic Radiated Power \\ EOA Energy Optimization Approach \\ ESS extended service set \\ FA Flow Augmentation
}


FDMA frequency division multiple access

kJ kilojoule

GPS Global Positioning System

IP Internet Protocol

LE Low Energy

MAC medium access control

MCFA Minimum Cost Forwarding Algorithm

MIRS minimum input receiver sensitivity

MPR multiple packet reception

MST maximum sustainable throughput

NLOS non-line-of-sight

NPP Network Planning Protocol

PC personal computer

PDA personal digital assistant

PHY physical

QoS quality of service 


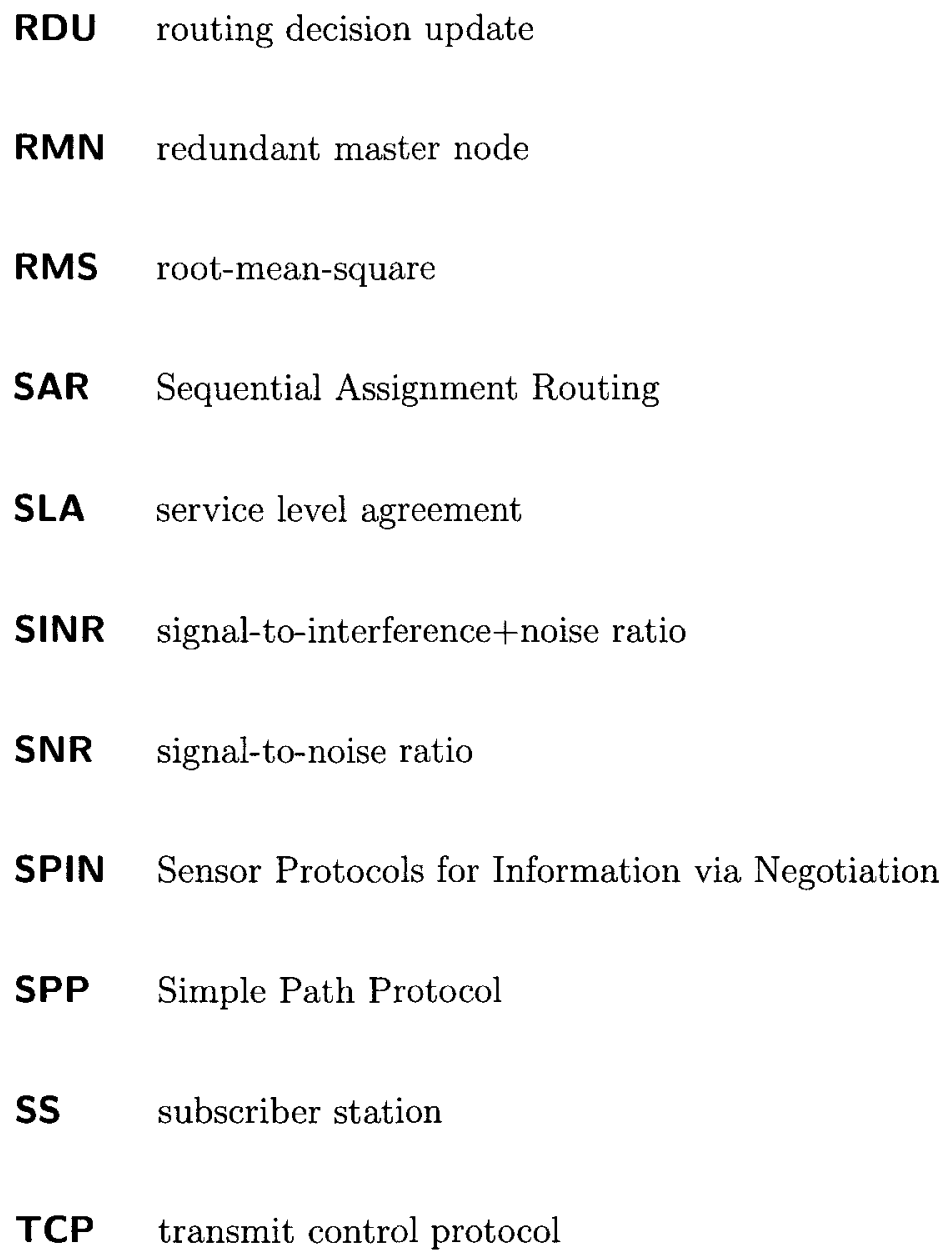

SLA service level agreement

SINR signal-to-interference+noise ratio

SNR signal-to-noise ratio

SPIN Sensor Protocols for Information via Negotiation

SPP Simple Path Protocol

SS subscriber station

TCP transmit control protocol

TDMA time division multiple access

TPC transmit power control

UWB Ultrawideband

WBAN Wireless Body Area Network

WiFi wireless fidelity 
WiMax Worldwide Interoperability for Microwave Access

WLAN Wireless Local Area Network

WPAN Wireless Personal Area Network

WSN wireless sensor network

WUSN wireless underground sensor network

xix 


\section{Chapter 1}

\section{Introduction}

The design of next-generation wireless sensor networks (WSNs) will require significant attention to be given to the performance of real-time applications. These applications consist of those for system control and resource management with varying resource and performance requirements. For example, these applications may include the monitoring of the structural health of building and bridge infrastructures, video monitoring, or maintenance of light and temperature levels. WSNs also have many military and safety applications such as the detection of enemy units, chemical agents and intruders. Given that many of these applications are core to system control, performance is also a major consideration to enable timely responses to critical events.

The consideration of application performance is complicated by sensors that have critical power constraints. As a result, the optimization of these networks traditionally takes the form of the minimization of energy consumption, or the maximization of network lifetime, in these networks as the primary objective [1][2][3][4]. However, 
this typically occurs to the detriment of application performance. Certain studies do strive to reach a maximum delay requirement [5][6][7]; however, it is unknown if we can do better as performance is not optimized. Other studies perform rate control in WSNs but do not model the power cost of using a transmission link in terms of the achievable throughput level [8]. Next-generation sensor networks require performance optimization by considering both the potential performance that can be achieved and the corresponding impact on a node's energy capacity. This approach shall enable nodes to make more informed resource allocation decisions.

In this dissertation, a cross-layer policy for joint throughput and energy optimization in these networks is presented. We derive the link suitability of a transmission channel, which determines the optimal physical (PHY) layer resource allocation that jointly maximizes achievable throughput and minimizes the associated power cost of using the link. This differs from current approaches that fit into one of two categories: those that perform rate control but do not model power cost in terms of the achievable throughput on the transmission link, or those that do not separate performance-related benefits and energy-related costs to ensure that we can jointly maximize throughput and minimize energy consumption. By maximizing link suitability, our method ensures that WSNs allocate limited network resources to transmission channels capable of a higher level of throughput and energy conservation.

Using link suitability in a cross-layer policy, we create a cross-layer optimization protocol that is unique in identifying routing paths with the optimal trade-off between throughput benefits and energy costs across multiple transmission channels. Our 
policy is based on transmit power at the PHY layer, channel allocation at the link layer and multi-hop path selection at the network layer. We also consider power consumption in the transceiver circuitry for both transmitters and receivers, and real-time residual energies of nodes. This enables us to create an adaptive protocol that responds to the real-time state of the network.

We also contribute the Network Planning Protocol (NPP) that derives the relationship between expected performance, network lifetime, message load and energy capacity for next-generation WSNs. This relationship enables us to balance network requirements to achieve both significant network performance and sustainable network lifetimes. This goal is achieved by deriving the NPP in terms of PHY layer resources that affect throughput and energy capacity. As a result, we are able to study the impact of various network design decisions, such as the choice of communication protocol or the frequency of presence announcements in the network, on our performance and network lifetime objectives.

We present joint throughput and energy optimization for distributed and application-centric WSNs. Application-centric WSNs refer to state-of-the-art sensor networks that execute applications such as video monitoring with significant performance requirements. These networks require performance optimization, but also major considerations for energy conservation schemes for sensors. We denote the current state-of-the-art in WSNs based on low data rate technologies such as Zigbee as non-application-centric, as they achieve low performance levels and have a limited range of applications for next-generation WSNs. 
In the future of system control and resource management, it will also be necessary in several instances to deploy one or more sensor clusters to execute multiple sensory applications simultaneously. We expect that these applications will have varying bandwidth requirements. For example, a controller on a university or office campus requires multiple sensor clusters to monitor temperature in a number of computer server rooms. Meanwhile, other sensor clusters perform video monitoring of high security areas. Additional sensor clusters measure the variations in ambient light on various building floors to automatically adjust light levels to save energy costs. This control system requires an architecture that enables the efficient feedback of diverse sensory data from geographically distributed clusters to one or more processing centers to manage resources or respond to critical events. As a result, we are exploring the use of mesh networks to enable the creation of a distributed WSN architecture.

\subsection{Wireless Mesh Networks}

A wireless mesh network is a type of wireless ad-hoc network where nodes are organized in a mesh topology and are typically connected in a fully-connected or partiallyconnection fashion. This provides reliability and redundancy by offering many communication options in the event that one node fails and can no longer operate.

This study utilizes two types of wireless nodes, mesh nodes and sensor nodes. Mesh nodes in our study are located at sensory locations of interest and are assigned to govern sensor clusters as their cluster-heads. Given the increased size of mesh 
nodes in comparison to sensors, they can be deployed with more resources such as more memory, a better central processing unit (CPU) and a larger battery than sensors in the network. As a result, these mesh nodes can assume the computational responsibilities for optimization, reducing the strain on limited sensor energies. Given the terrain or environment in which the sensor network is deployed, certain mesh nodes may be connected to power sources if the locations in which the mesh nodes are deployed have power sources readily available. Alternatively, we may be able to recharge the energies of mesh nodes with solar or light sources to replenish their energy reserves over time. Given these options, mesh nodes are well-suited to perform resource allocations on behalf of sensors in the network. However, even if power or light sources are not available at the specific location for a mesh node, the mesh node's larger size enables them to be deployed with more resources than sensors. This enables mesh nodes to provide centralized control such that we may reduce the sophistication of sensors.

The cluster-head itself is responsible for two functions. Given that the clusterhead is assigned to a cluster, it governs the operation of the sensors in its subsection of the sensor field by optimizing their resource allocations via cross-layer optimization. By leaving the computational responsibilities for the cluster with the cluster-head, a greater percentage of sensor energy can be used for sensing and communication, as opposed to computation. The second function of the cluster-head is to accumulate information from the sensors in its cluster and communicate that information to the master node. Given that the sensor cluster and mesh network will likely differ in 
technology, the cluster-head will use software radio to convert packets arriving from a sensor cluster prior to transmitting those packets through the mesh network.

The overall network is governed by a master node, which in our network is a mesh node that is responsible for gathering data from the cluster-heads in the network before transmitting that data to a processing center for analysis. The master node either transmits the information over the Internet to network operators who analyze the information provided to perform maintenance functions, or act as repositories for collected information and perform automated functions on behalf of the operator. In this work, the master node is the destination for all data packets.

\subsubsection{Wireless Sensor Networks}

WSNs are one form of multi-hop mesh network that consist of groups of hundreds or thousands of small, autonomous sensing nodes. These nodes act as both end nodes and intermediate relay nodes, and can be organized in a star or mesh topology, or in an ad-hoc fashion. A typical sensor network is presented in Figure 1.1. Each node consists of a short-range wireless transmitter/receiver, sensing unit, storage unit and processing unit [9], while they are also typically battery-powered, and have low computational capabilities and limited memory. Hence, sensors typically lack the sophistication necessary to govern the sensor field. Paths in WSNs are typically multihop to reduce the range over which a sensor must transmit, but a greater number of sensors relaying traffic also reduces the overall remaining network energy. 


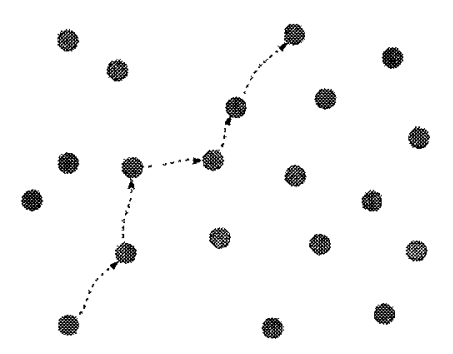

Figure 1.1: A Sensor Network with Multi-Hop Paths

With limited resources, energy conservation is crucial. As a result, sensors generally sleep in low power states during periods of inactivity. It may also be possible, given certain applications that do not require immediate response, to store gathered sensory data in memory and transmit all the data at once to reduce the number of required sensor transmissions. For example, sensor measurements of light or temperature levels may be taken every few seconds but may only transmit data once a minute. However, considerations must also be made for the limited memory of sensors.

Groups of sensor nodes are also meant to operate in a self-organized fashion and, thus, the operation of WSNs has spawned analogies to the collaboration and synergistic behavior within ant colonies [10] [11]. Computational tools such as swarm intelligence have also been developed to implement this self-organization [12].

\subsection{State-of-the-Art in Mesh Networks}

Significant research is being conducted in next-generation WSNs based on high speed technologies such as Ultrawideband (UWB) and Bluetooth. EnOcean also offers 
battery-free sensors and radio switches that perform energy harvesting to build selfpowered WSNs [13][14]. Research by the IEEE 802.11s Task Group to include medium access control (MAC) and PHY layers for mesh networks is also an attractive option for overlay design. However, prior to building and analyzing next-generation wireless mesh networks, a survey is needed of available mesh technologies.

\subsubsection{Communication Technologies for Mesh Networks}

In application-centric WSNs, the selection of the communication technology used for the wireless links is crucial. To date, the 802.15.4 Zigbee standard has typically been used in sensor networks and is inherently a low date rate technology. However, several IEEE standardization groups are working, or have worked, to include wireless mesh network specifications into their standards [15]. These include those for IEEE 802.11s, IEEE 802.15.5, IEEE 802.16a and IEEE 802.20, all of which have had tasks groups assigned to define requirements for mesh networking and are all at different stages in their maturity [15]. The 802.11s standard is looking to extend the IEEE 802.11 architecture to provide extended service set (ESS) mesh, in enabling access points to establish wireless links among each other to perform dynamic path configuration [15]. This would also allow for the creation of neighborhood networks [15]. In July 2010, the 802.11s Task Group approved a motion to approve the latest draft in support of a request to forward the draft 802.11s to sponsor ballot [16]. The First Recirculation Sponsor Ballot and Second Recirculation Sponsor Ballot were closed on January 10, 
2011 and March 5, 2011, respectively, both with 95\% approval rates [17]. As of April 15, 2011, some Third Recirculation Sponsor Ballot comments were available on the documents server of IEEE 802.11 [17].

The IEEE 802.15.5 is working to establish short-range connectivity for small groups of fixed and mobile devices, such as consumer electronics, cell phones, personal computers (PCs) and personal digital assistants (PDAs). The use of mesh networking for the 802.15 .5 group is motivated by the power limitations of mobile devices. Employing mesh-like multi-hopping communication increases the coverage of wireless personal area networks (WPANs) and allows devices to communicate over shorter links, thus preserving power. Doing so provides higher throughputs, fewer retransmissions and is better suited to the propagation characteristics of UWB [15].

The IEEE $802.16 \mathrm{a}$ standard was ratified in January 2003 to provide point-tomultipoint non-line-of-sight (NLOS) operations and, thus, allowed 802.16a standard mesh extensions to be included in the specification. If operating in mesh mode, a subscriber station (SS) may have direct links to other SSs, and data traffic can be routed over multiple hops to the destination SS. Finally, the IEEE 802.20 Working Group intends to provide mobile broadband wireless access over the mesh networking paradigm at vehicular speeds up to $250 \mathrm{~km} / \mathrm{h}$ in licensed bands below $3.5 \mathrm{GHz}$ [15].

Cheekiralla and Engels [20] analyze communication technologies for WSNs by developing a classification scheme for WSN devices. Table 1.1 compares a number of these technologies, among others, with respect to data rates and operating frequencies. Zigbee is a low-data rate technology for wireless monitoring and control 
Table 1.1: Typical Data Rates and Frequency of Operation for Different Standards [14][18][19]

\begin{tabular}{ccc}
\hline \hline Standard & Typical Data Rate & Frequency of Operation \\
\hline Bluetooth Classic & $2.1 \mathrm{Mbps}$ & $2.4 \mathrm{GHz}$ \\
Bluetooth High Speed & $24 \mathrm{Mbps}$ & $2.4 \mathrm{GHz}$ \\
Bluetooth Low Energy & $1 \mathrm{Mbps}$ & $2.4 \mathrm{GHz}$ \\
EnOcean & $125 \mathrm{kbps}$ & $868 \mathrm{MHz}$ or $315 \mathrm{MHz}$ \\
ZigBee & $250 / 40 / 20 \mathrm{kbps}$ & $2.4 \mathrm{GHz}$ or $915 \mathrm{MHz}$ or $868 \mathrm{MHz}$ \\
UWB & $480 \mathrm{Mbps}$ & $3.1-10.6 \mathrm{GHz}$ \\
WiFi 802.11a & $54 \mathrm{Mbps}$ & $5 \mathrm{GHz}$ \\
WiFi 802.11b & $11 \mathrm{Mbps}$ & $2.4 \mathrm{GHz}$ \\
WiFi 802.11g & $54 \mathrm{Mbps}$ & $2.4 \mathrm{GHz}$ \\
WiMax 802.16-2004 & $75 \mathrm{Mbps}$ & $5.25-5.725 \mathrm{GHz}$ \\
\hline
\end{tabular}

systems. While Zigbee has a range of approximately 10-100 meters, it was ratified for use in ad-hoc and peer-to-peer networks specifically, and thus has been popular in developing initial WSNs. However, with an achievable data rate of $250 \mathrm{kbps}$, the current generation of Zigbee radio is unable to carry high-speed traffic in WSNs. On the other hand, an analysis of the power consumption of Zigbee compared to other communication technologies given in Table 1.2 demonstrates that Zigbee is among the lowest in energy consumption among its competing technologies. With that said, given its performance limits, Zigbee is not suited to application-centric WSNs.

An alternative to Zigbee radio is UWB which occupies the spectrum from 3.1 to 10.6 $\mathrm{GHz}$ at a level at or below $-41.3 \mathrm{dBm} / \mathrm{MHz}[21]$ and currently reaches data rates of roughly $50-100 \mathrm{Mbps}$ with achievable rates of $500 \mathrm{Mbps}$ in the future [18]. However, UWB does not refer to any particular technology or radio type, but rather only to the available spectrum [22]. Thus, many technologies will occupy UWB spectrum. Due 
Table 1.2: Energy Efficiency Coefficients for Various Standards [2][23]

\begin{tabular}{cc}
\hline \hline Standard & Energy Consumption Per Payload Bit \\
\hline Bluetooth Classic & $2.5 \mu \mathrm{J}$ \\
ZigBee & $0.5 \mu \mathrm{J}$ \\
UWB & $0.01 \mu \mathrm{J}$ \\
WiMax & $7.0 \mu \mathrm{J}$ \\
\hline
\end{tabular}

to the rates being discussed, devices based on UWB are also inherently short range, suitable for many applications for sensor networks but require significant multi-hop routing. Also, if we observe the energy efficiencies for these technologies in Table II, we see that UWB also has the lowest energy consumption per payload bit. As a result, the use of UWB WSNs allow us to achieve high-speed sensor networks that are energy-aware and show significant promise for state-of-the-art WSNs. However, the design of UWB devices is complex due to the need to design transceivers to operate over a wide band.

An interesting development in the last few years has been the emergence of EnOcean, a German energy-harvesting technology for WSNs [13][14]. EnOcean products include energy-harvesting wireless sensors and switches that contain miniaturized energy converters that harness energy from motion, or changes in temperature, light or heat, whenever sensors take measurements [14]. They harness sufficient energy to transmit wireless signals without the need for batteries. These products also contain ultra-low power electronic circuitry and wireless transceivers for the deployment of maintenance-free and self-powered WSNs for industrial or home automation [14]. EnOcean technology uses the $868 \mathrm{MHz}$ and $315 \mathrm{MHz}$ frequency bands and can achieve 
low data rates of $125 \mathrm{kbps}$ [14]. As a result, they achieve lower rates than Zigbee, but provide significant benefits for low power WSNs. However, the applications for high capacity WSNs are naturally restricted given the low achievable data rates. EnOcean is assembling the EnOcean Alliance of leading companies worldwide to standardize and promote innovative control solutions [13]. A performance analysis of the EnOcean protocol is presented in [24], while other studies have also explored energy harvesting in $[25][26][27][28]$.

The Bluetooth Special Interest Group adopted the Bluetooth version 4.0 standard in June 2010 [19]. Bluetooth v4.0 consists of three protocols: Bluetooth Classic, Bluetooth High Speed and Bluetooth Low Energy (LE); manufacturers of devices are expected to implement Bluetooth LE and subsequently choose the class or high speed modes to build on top of it. Bluetooth LE provides an interesting alternative to Zigbee for low-power networks. However, while applications will likely overlap between these technologies, they will likely exist in their own niches. Bluetooth LE maintains the synchronous connection from its Bluetooth predecessors that requires a hand-shaking between devices [19]. As a result, it will likely become more prominent in WPANs and wireless body area networks (WBANs). Since Zigbee maintains asynchronous communication, it will likely remain as an alternative to Wireless Local Area Networks (WLANs) and mesh networks. For WSNs specifically, while Bluetooth LE seems promising as it achieves significantly higher data rates with low power, Bluetooth applications cannot access information necessary for synchronization and device discovery that has been the basis of the protocol since its inception [20]. At the time of 
the writing of this dissertation, Bluetooth LE devices have been available for roughly six months and, while Bluetooth LE is meant to provide energy savings of ten times or more over Bluetooth Classic [19], the standard energy consumption coefficient for Bluetooth LE devices has yet to be published.

\subsubsection{High-Speed Mesh Networks}

Much research has been done in using high-speed wireless technologies such as UWB to build high-speed WSNs as an alternative to Zigbee networks. One study uses UWB WSNs to build a low complexity solution for outdoor applications for the transmission of location and tracking information. In this study, Oppermann et al. [2] use a wide deployment of low-power and low-cost sensor devices that are worn by several users, typically athletes across a facility to track both location and performance metrics. By taking advantage of the favorable time domain resolution characteristics of UWB, Opperman et al. use UWB' devices worn by cross-country skiers to relay positioning and performance information for each of these users via peer-to-peer connections to fixed nodes in the network for processing.

If these users are out of range of one of the fixed nodes, the user devices send their information across multi-hop paths through other user devices that act as intermediate relay nodes. Oppermann and Yu publish results for the root-mean-square (RMS) error of the position coordinates and failure rate using quasi-Newton methods within a monitoring area with dimensions $90(1) \times 90(w) \times 4(h)$ metres [3]. While this study 
demonstrates reasonably favorable results, it points to the large bandwidth of the UWB signal to distinguish many multi-path components to increase both the positioning accuracy and diversity to exploit the available capacity [2]; this is highly beneficial in sensor networks to increase the achievable capacity and determine the position from which a measurement was taken. However, the fixed nodes in this study that relay information to the central processor are connected via cables to a central processor. Laying cable to connect fixed nodes is not always possible where WSNs are deployed, such as for military applications or for wide-scale deployment of WSNs to monitor the health of civil infrastructure. In fact, for some applications, the cost of laying cable can be $30-45 \%$ of the total monitoring installation cost [4]. As a result, while this work is useful, it may not be a practical model to use for our study.

Several other studies analyze the UWB WSN for smaller research problems. One such study by Mehta and El Zarki designs a MAC protocol for UWB WSNs [4]. They focus on creating a topology formation and performing data aggregation in their study to use UWB WSNs in the monitoring of the health of civil infrastructure such as highways and bridges [4]. For their MAC protocol, they utilize a time division multiple access (TDMA) mechanism and a sink that is assumed to have unlimited amounts of energy and computational power. In their study, it is the sink's responsibility to execute their optimization algorithm to determine the routing paths from sensors as well as the transmission schedules of the sources of the data. This is a positive step as it takes the computational responsibilities away from ordinary sensors in the network to allow them to focus on sensing and routing as opposed to computation. 
The major shortcoming of their optimization algorithm is an assumption that intermediate nodes between the source and sink are only considered as relay candidates if the distance between the prospective relay node and the sink is less than the source's distance to the sink [4]. This is not a realistic assumption in the optimization of the network lifetime. It is possible that a path through nodes with higher residual energies through intermediate nodes, which are not necessarily closer to the sink than the source, may lead to greater network performance. This may also avoid further draining of the energies of nodes between the source and sink by not using the same nodes in the routing process repeatedly, although Mehta and El Zarki do consider the energies of sensors during the routing decision [4]. However, by making this critical assumption and reducing the set of potential neighbors, paths are selected from a smaller set of candidate paths and, hence, will be used more frequently than if the set of potential neighbors was larger. In fact, in many cases, the source itself may be severely constrained for energy resources and a simple hand-off to a nearby node that may be slightly further from the sink, may lead to a far more optimal path. As a result, from our perspective, this routing algorithm is sub-optimal.

A third study on UWB-based sensor networks by Shi et al. studies the routing problem in relation to the capacity of these networks [29]. They study the problem of whether or not it is possible that, if a set of source nodes all transmit at different data rates to a base station (BS), all rates are relayed successfully considering the maximum bit rate that the $B S$ can receive. They suggest that, due to interference limits, even if the sum of bit rates is less than the bit rate limit that the BS can 
receive, the network may be unable to support these rates due to interference, the topology of the network, the bit rates themselves and other network characteristics.

Shi et al. devise a cross-layer optimization approach and a fast heuristic algorithm to study this feasibility problem by analyzing link-layer scheduling, routing in the network layer and transmit power control (TPC) [29]. The feasibility factor, $K$, is defined as the ability of the algorithm to find a multi-hop route at the network level, and a scheduling policy at the link layer; a power control strategy is also developed at the link layer that corresponds to bit rates. Given that the UWB spectrum of 7.5 $\mathrm{GHz}$ bandwidth has a minimum channel size of $500 \mathrm{MHz}$, the algorithm is analyzed for $1 \leq M \leq 15$ channels (sub-bands) and the feasibility factor $K$ is analyzed for different $M[29]$. As $M$ increases, they confirm that the feasibility factor $K$ is a nondecreasing function of $M$ because nodes can tune their frequencies to a greater number of channels to avoid interference and increase capacity [29]. The result to highlight is that their optimal routing policy using cross-layer optimization outperforms both minimum energy routing paths and minimum hop routing paths [29].

Another interesting technology to consider for WSNs is 802.11 WLAN. While there are many flavors, including $802.11 \mathrm{a}, \mathrm{b}, \mathrm{g}, \mathrm{n}, \mathrm{k}$, s, etc., a feasibility analysis indicates that, although high date rates and long ranges can be achieved quite readily with 802.11 (as has been done in home networking), the power consumption of 802.11 is not suitable for WSNs. However, the IEEE 802.11s Task Group is working to include MAC and PHY layers for mesh networks to improve the coverage of WLANs and, thus, 802.11 s may be a suitable technology for overlay mesh networks [30]. 


\subsection{Motivation}

Given these state-of-the-art technologies, among others that will emerge in the future, WSN applications will be plentiful. In the future of system control and resource management, next-generation WSNs shall be large networks composed of geographically distributed sensor clusters that execute many of these applications simultaneously. These sensor clusters shall be connected to processing centers via an overlay network in a distributed architecture. In our study, the overlay network is a mesh network that forms a communication backbone for the feedback of diverse application data from sensor clusters through a cluster-head to the processing center. As has been mentioned, cluster-heads are nodes in the mesh network that are assigned to govern a sensor cluster and act as a bridge to the mesh network for sensor transmissions. Since the range of sensor network applications will be diverse, they will also have varying resource and performance requirements. As a result, it shall be imperative to optimize these networks both in terms of throughput and energy conservation. These networks are called application-centric WSNs, and pose several research challenges that merit investigation and provide the motivation for performing this study.

\subsubsection{The Throughput Problem}

Next-generation WSNs will be application-centric and require major considerations related to throughput and energy conservation. It will be expected in future networks 
that applications are running at peak performance, especially in WSNs that are providing feedback of critical data related to resource management. However, the current state of WSNs are far off from this standard given the critical power constraints that sensors face. In order to optimize both throughput and energy conservation, it shall be important to study physical resource consumption. More specifically, modeling the trade-off between application performance and energy consumption in terms of these resources should be the prime focus of optimization algorithms. In this manner, we may study resource allocations in these networks and ensure that nodes are allocating limited resources optimally to transmission channels that are capable of achieving a higher level of performance and energy conservation. As a result, in this thesis, we tackle the performance and energy conservation issue by maximizing the trade-off between throughput and energy consumption in terms of key physical resources, such as transmission power, that impact a node's energy capacity.

\subsubsection{Optimization in Software Radio Systems}

In WSNs that are based on multiple technologies, software radio is required to convert operating parameters between otherwise incompatible communication protocols. For example, the cluster-head must convert the communication parameters between the wireless standards used in the sensor and the mesh networks to enable communication. In the optimization of these systems, this conversion must consider the varying dependencies of these technologies on a number of characteristics that affect 
communication and performance. These characteristics include:

- Energy efficiencies of the radio used in the sensor and mesh devices,

- Operating bandwidths,

- Transmit power levels, and

- Achievable throughputs.

With the use of utility functions that use the above parameters and form the objective functions for optimization of these networks, the operation of multiple technologies in these systems must be tuned appropriately through careful parameter selection. Otherwise, resource hoarding may result where nodes of one technology overuse resources leading to shorter node lifetimes. As a result, this is an important research consideration in multi-technology systems for network design.

WSNs that are based on software radio also enable for the deployment of sensor clusters and a mesh network that are designed with technologies that are most suitable to their applications. Various technologies may be selected based on throughput requirements, cost of deployment and energy efficiency. However, given applications that have desired sensory locations of interest, considerations must be made for the required density of the network since different communication technologies limit the distance between nodes. Software radio provides compatibility between these technologies, while it would also be used to dynamically tune operating parameters around current networking conditions to improve capacity. 


\subsubsection{Overlay Mesh Networks as an Enabling Technology}

Mesh networks are used in our study to connect sensor clusters that are distributed geographically to a master node for the feedback of sensory data. However, with the use of a mesh network, this adds a second stage of optimization to our problem. While in the first phase, our system must optimize over the sensor portion of a path to the cluster-head, our algorithm must subsequently optimize the transmission to the master node over the mesh portion of a path. This is due to a design decision made in our study that mesh nodes are not wired to power sources; this enables us to maintain a flexible architecture where mesh nodes may be deployed in locations that may not have power sources readily available. Thus, in our study, they do not have unlimited resources available and require optimization, which adds additional signaling and resource requirements to our problem. As has been mentioned, in practice, it may be possible to recharge the energies of mesh nodes with solar or light sources to replenish their energy reserves over time. However, we do not apply this approach to the formulation of our problem as the limited life span of the mesh network enables us to test its ability to survive for a predictable network lifetime.

The advantages of using overlay mesh networks are four-fold. Firstly, and the most obvious, the mesh network enables the formation of the distributed WSN architecture by connecting distributed sensor clusters to a master node over a large area. In this way, multiple sensor clusters can share the mesh network for long-haul routing. Without the mesh network, this architecture may not be realizable. The mesh network 
also allows us to deploy a sensor network in remote areas where fibre may not be available. It would only be required to deploy a mesh node to extend the range of the mesh network to the geographical area of interest after which the mesh node acts as a cluster-head to govern the sensor cluster. Thus, in locations where Internet Protocol (IP) traffic cannot be carried, mesh technologies can be used to provide communication and feedback of sensory data to the processing center. However, it should also be noted that the deployed mesh network may be used to carry multiple types of traffic, only one of which may be sensory data.

Secondly, the mesh network provides an outlet for a sensor cluster in the form of a mesh node that reduces the routing burden on resource-constrained sensors. Rather than using significant multi-hop routing across short-range nodes, the mesh network only requires that a source sensor routes traffic to its nearest mesh node (the clusterhead). This reduces the number of sensor nodes involved in the routing process. We propose that, by reducing the number of messages routed by sensors along a path, a greater portion of sensor energy will be used for sensing and computation as opposed to communication. Moreover, it enables for the deployment of sensor networks that execute diverse sensory applications that are more resource intensive.

Thirdly, the mesh network provides a communication option in the form of a solid mesh backbone with several point-to-point links for link reliability. Thesc links can be used by distributed sensor fields to transmit information to the master node.

The fourth advantage of using overlay mesh networks is that mesh nodes, given their increased size in comparison to sensors, can be deployed with greater resources 


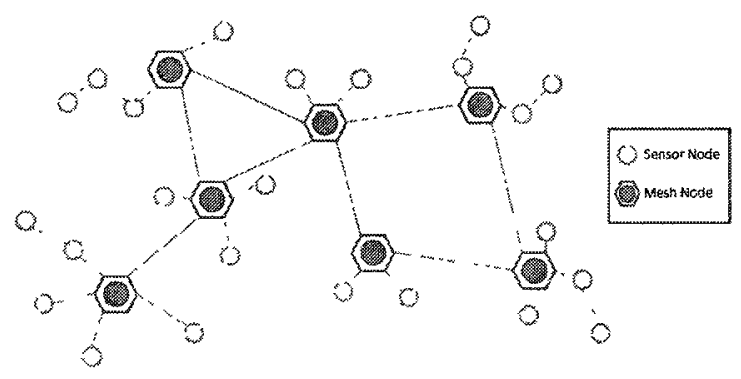

Figure 1.2: A Two-Tier Multi-Hop Sensor Network Architecture

than sensors in the network. As a result, these mesh nodes can assume the computational responsibilities for optimization, further reducing the strain on limited sensor energies. An example of a two-tier sensor network is presented in Figure 1.2.

\subsubsection{Cross-Layer Design in WSNs}

Layering systems are the norm in the design of communication protocol stacks. However, wireless systems are not always suited to the common layered protocol stack architecture. For example, in a layered architecture using transmit control protocol (TCP), a failed packet is considered a sign of congestion, as opposed to simply a lost or corrupted packet which is the case in wireless systems. For sensor networks specifically, given the need to conserve sensor energy and maximize application performance, cooperation between several layers in the protocol stack is crucial, which can only be achieved in a cross-layer architecture. Cross-layer design is necessary more specifically to ensure that, not only can the most energy-aware route be utilized to carry traffic, but the availability of energy and PHY resources can be considered in the throughput optimization problem. 


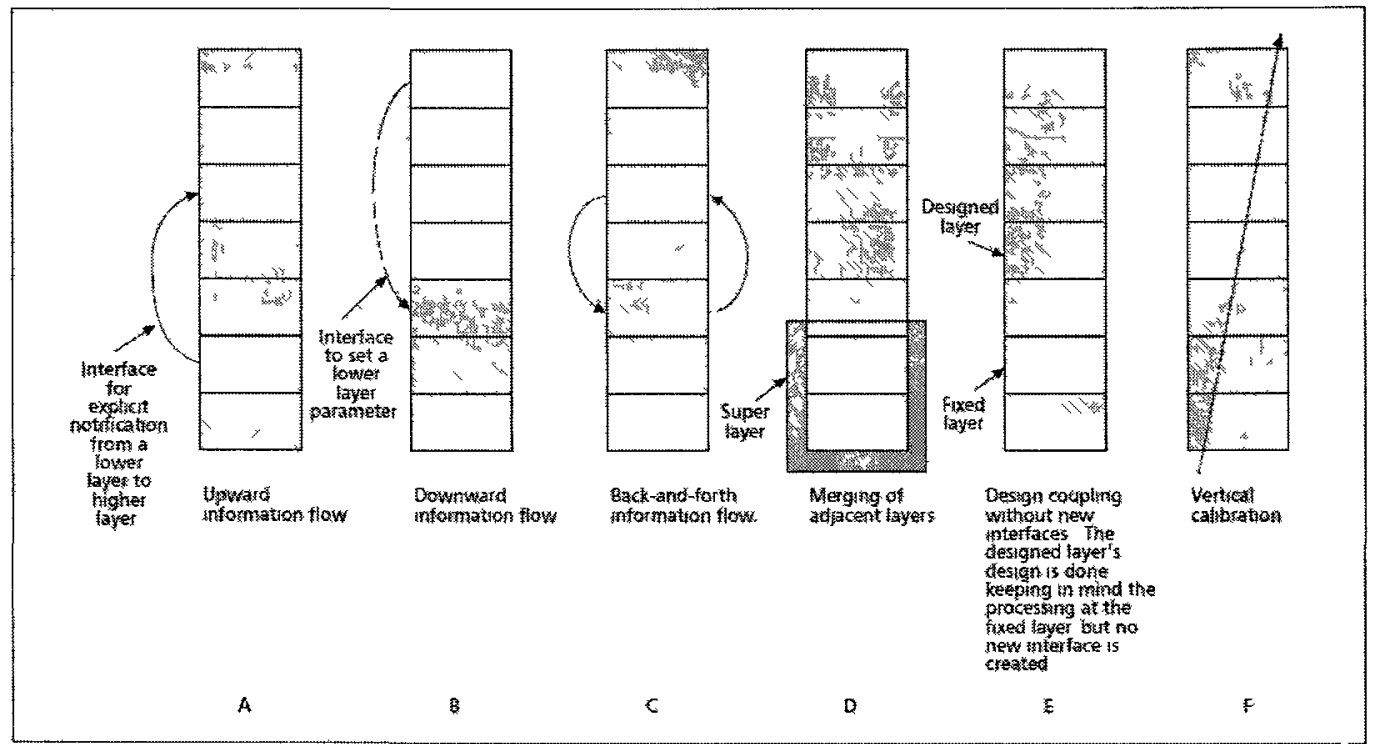

Figure 1.3: Various Cross-Layer Design Protocols [31]

Figure 1.3 presents a number of general ways in which a typical layered architecture can be modified by cross-layer design:

- Creation of new interfaces for information flow between non-adjacent layers (Figures 1.3a-c),

- Merging of adjacent layers for joint functionality and reduced overhead (Figure $1.3 \mathrm{~d})$

- Design coupling between layers, i.e. one layer assumes information arriving from another (Figure 1.3e); and,

- Vertical calibration between layers (Figure 1.3f).

In application-centric WSNs, a number of these protocols may be used For example, upward information flow (Figure 1.3a) may be used to provide the application 


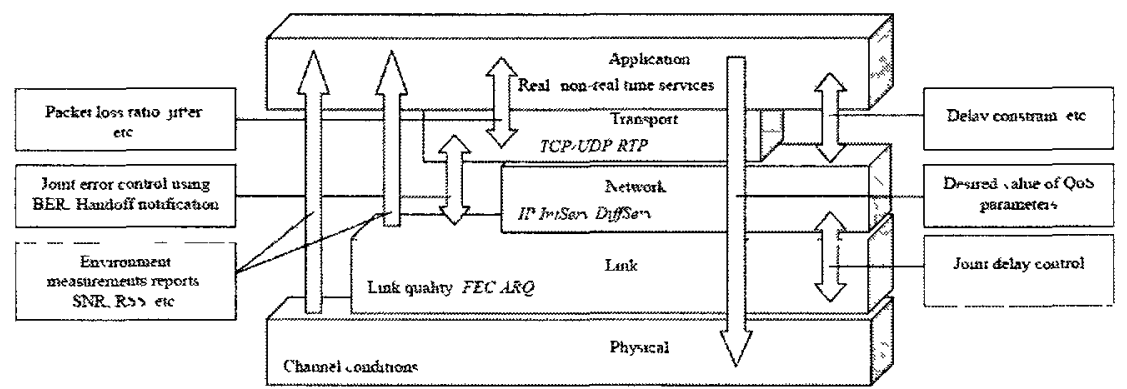

Figure 1.4: CLASS, a proposed cross-layer stack for wireless systems [32]

layer with available routes from the network layer, channel availability from the link layer and remaining energy information from the PHY layer. Furthermore, downward information flow (Figure 1.3b) or back-and-forth information flow (Figure 1.3c) may be used between the application layer and the PHY layer. For example, the application layer may inform the PHY layer of transmit parameters such as transmit power and operating frequency for resource allocation.

A proposed cross-layer protocol stack for next-generation wireless systems known as CLASS (Cross-LAyer Signalling Shortcuts) is presented in Figure 1.4 to provide coordination and reduce delay between multiple layers [32]. This reduction in delay is crucial to improving the response times for applications in next-generation WSNs. We present our cross-layer design in Chapter 4.

\subsection{Problem Statement}

This thesis explores the throughput and energy optimization problem via cross-layer design for distributed and application-centric WSNs. The six goals of this research 
are as follows:

1. Define the distributed WSN architecture that is enabled by wireless mesh networks. This architecture is defined having reviewed the state-of-the-art in crosslayer optimization of WSNs and acknowledged the need for such an architecture;

2. Quantify link suitability in terms of throughput benefits and energy costs as a function of PHY layer resources such as transmit power and frequency. The link suitability is the profit gained by using a link in a path;

3. Form the cross-layer optimization policy to meet the needs of next-generation WSNs in terms of joint optimization of the PHY, link and routing layers. The optimization algorithm determines the optimal path from a source sensor to the master node, with the highest trade-off of throughput to energy consumption along each hop. The PHY layer optimization maximizes the link suitability and allocates transmit power to achieve the optimal level of throughput. At the link layer, channel scheduling occurs to maximize capacity and reduce interference. Path selection at the routing layer scales link suitabilities by residual energies to avoid nodes with lower resource availabilities;

4. Design a NPP to simplify the design process for next-generation WSNs. The NPP quantifies the relationship between expected performance for an application, desired network lifetime, message load and energy capacity for sensors and mesh nodes. This enables network planners to deploy next-generation WSNs 
that do not simply maximize network lifetime or minimize energy consumption to the detriment of application performance. Rather, the protocol simplifies network planning by allowing us to evaluate the impact of various network design decisions on both performance and network lifetime objectives;

5. Implement survivability protocols for next-generation WSNs that enable the network to recover from changes in network connectivity that stem from failures, intrusions or attacks. This requires the network to be able to recover from lost nodes and links, and re-allocate resources accordingly; and,

6. Design a priority scheme for distributed WSNs that enables for one or more clusters to be placed on high priority at any time. Given that sensor clusters are geographically distributed and the network executes multiple sensory applications simultaneously, it is likely that, at any time, one or more events require immediate attention. Clusters placed on high priority pre-empt active transmissions and are allocated optimized resources immediately for rapid response.

\subsection{Contributions of this Research}

This thesis has three main contributions:

1. The link suitability method to quantify the optimal PHY layer resource allocation that achieves joint throughput and energy optimization in applicationcentric WSNs. By maximizing the trade-off between achievable throughput 
benefits and the associated power cost of using a link, we ensure that nodes are allocating resources optimally to transmission channels that are capable of a higher level of throughput and energy conservation;

2. A cross-layer optimization policy that is unique in identifying routing paths in WSNs with the optimal trade-off between throughput benefits and energy costs across multiple transmission channels. Our policy is based on: i) path selection at the network layer; ii) channel selection at the link layer; and, iii) transmit power based on the link suitability at the PHY layer; and,

3. A NPP that contributes the relationship between expected performance, network lifetime, message load and energy capacity for application-centric WSNs. The NPP enable network designers to study the impact of various network design decisions on performance and network lifetime objectives.

\subsection{Outline of Thesis}

This thesis comprises of seven chapters. Chapter 2 presents the literature review that lead to our acknowledgement of the throughput and energy problem in nextgeneration WSNs, and the definition of our distributed WSN architecture. The details of our problem formulation, formal problem definition and objective function are presented in Chapter 3, while Chapter 4 presents our cross-layer design. Chapter 
5 discusses system implementation issues including system design, parameter selection, simulation methodology and performance metrics. Performance results of our application-centric and distributed WSN are presented in Chapter 6, while we conclude in Chapter 7 outlining future directions for research. 


\section{Chapter 2}

\section{Background}

\subsection{Introduction}

The design of a cross-layer optimization policy to perform throughput and energy optimization in application-centric WSNs draws knowledge from many different areas. Firstly, a thorough review of current research in the cross-layer optimization of WSNs, considerations that have been made for throughput performance in related work, and existing approaches for network planning will be required. Secondly, cross-layer optimization in our study requires knowledge of preference modeling from microeconomic theory. Preference modeling enables us to model the variation in value placed on our resources of interest by transmitters and receivers in the network. This is an important consideration in WSNs because our network is heterogeneous in modeling several wireless technologies; different technologies have varying trade-offs between power, bandwidth and data rate that must be quantified. In addition, since mesh nodes are less constrained for resources than sensor nodes, sensor nodes and mesh 
nodes value their resources differently which should also be considered. Modeling these trade-offs mathematically, determining methods of quantifying the benefits and costs to evaluate these trade-offs appropriately, and maximizing preference are crucial to finding the optimal solution. We can also model the benefits and costs of resource consumption to study the impact of the consumption of PHY layer resources on a node's performance, energy capacity and lifetime.

Further issues require attention in building next-generation WSNs that are survivable. Routing protocols such as energy-aware routing that consider residual energy should be explored. Energy-aware routing allows us to vary which nodes are used in the routing process such that the power of certain sensors are not depleted excessively. By using the same nodes repeatedly in routing to the master node, we run the risk of network partitioning which is not desirable. Another form of implementing survivability protocols in WSNs is building redundancy into our network through the use of back-up cluster-heads and/or master nodes. These back-up mesh nodes reduce the computational load on mesh nodes that are responsible for handling the resource optimization and path assignment. These two methods allow us to build WSNs that can operate successfully in the presence of sensor and mesh node failure, malfunction or attack. Thus, current research into energy-aware routing will be reviewed, as well as current clustering and sensor network reconfiguration algorithms to reorganize the network to balance energy consumption.

Fourthly, it is important to review how the communication parameters of signal power, bandwidth and data rate relate to one another to determine the frequency 
division multiple access (FDMA) capacity. The communication parameters are the resources that shall determine the desired trade-off between throughput and energy consumption in the cross-layer policy. The energy consumption coefficient, which quantifies the energy consumption per bit for a technology, will also be reviewed and is crucial for comparing the energy efficiencies of various communication protocols.

These issues significantly impact the design and performance of our cross-layer optimization policy. As a result, this chapter will present material from a range of sources to develop the necessary background for these topics, all of which are crucial to developing an understanding of our WSN problem.

\subsection{Literature Review}

The performance limits of WSNs are studied in [33]. In this paper, $\mathrm{Hu}$ and $\mathrm{Li}$ aim to understand the fundamental limits on the maximum sustainable throughput (MST) of the sensor network, and the maximum active lifetime of the network [34]. Hu and $\mathrm{Li}$ define the active lifetime as the point at which the total energy consumed in the network equals the initial energy budget available at the start. It is defined at this point, beyond which, sensor network performance begins to decrease [33].

$\mathrm{Hu}$ and Li specify two constraints on capacity in WSNs: interference-constrained capacity and energy-constrained capacity [33]. Interference-constrained capacity is caused by two factors. The first is defined by Gupta and Kumar as the spatial concurrency constraint [35]: the phenomenon by which capacity limits are reduced 
due to more than one nearby network node attempting to transmit at the same time. Since signals at the receiver can be corrupted as a result, the network MST is reduced [33]. To reduce the effect of the spatial concurrency constraint, studies like those by Arms et al. have looked at frequency-agile WSNs to reduce the burden of co-channel interference in sensor networks [36]. Another study by Galbreath et al. observes the channel allocation problem in frequency-agile WSNs [37]. In fact, in 2004, companies such as MicroStrain, Inc. began manufacturing frequency-agile wireless sensors [36] that were patented in 2010 [38]. Another option is the use of UWB as an overlay technology to share spectrum with spectrum incumbents by operating at low powers.

The second factor identified by Gupta and Kumar that causes interferenceconstrained capacity is the multi-hop traffic constraint: interference that is caused when traffic is relayed hop-by-hop towards the receiver [35]. This reduces the MST of the network because each successive hop re-transmits the same data in forwarding it to the destination. This creates additional interference with other incoming packets to that node, reducing the achievable capacity. Gupta and Kumar also derive that per-node capacity is reduced by increasing the number of nodes in the network [35].

\subsubsection{Link Suitability and Throughput Modeling}

A number of studies have explored cross-layer optimization protocols specifically with throughput considerations in WSNs. One study by Yin et al. [8] performs joint transport and PHY layer optimization for rate and power control in WSNs. They seek 
the transmission rates that both maximize network throughput and network lifetime by modeling a utility function based on an aggregated data rate for a path as opposed to per-hop. The network lifetime consideration is made by minimizing the average power consumption due to transmission over all flows instead of considering the power consumption of the achievable rate itself. Link suitability should model the trade-off between achieving a higher transmission rate and the energy cost associated with the transmission rate, which is the approach to joint throughput and energy optimization in this thesis. In this fashion, we ensure that the resources are allocated efficiently to the transmission. Yin et al. also do not consider the routing and MAC layers. Sleep states are also not considered, while it is well known that a large portion of the network lifetime will be spent by sensors in low-power sleep states [39]. Furthermore, communication and routing protocols are not shown in [8], and the results do not show the ability of the algorithm to meet both significant throughput performance and a desired network lifetime.

Another study by Crichigno et al. in [40] studies the throughput problem as a joint routing and scheduling problem for WSNs that support multiple packet reception (MPR). They seek to maximize the aggregated throughput for the end-to-end flow subject to the optimal schedule of relay nodes over an $N$-hop path. PHY layer considerations are not made and, as a result, energy conservation that is critical to WSNs is not studied.

Krogsveen et al. also propose a cross-layer route optimization algorithm in [6] that minimizes the total energy consumption along a path subject to satisfying maximum 
delay and bit error rate (BER) constraints. Link suitability is quantified in terms of PHY layer parameters of modulation order and transmit power. However, minimum transmission rates are considered that meet delay constraints for quality of service (QoS). They also observe the routing problem by only assuming a single path of $L$ hops from source to sink. This poses a problem in large multi-hop WSNs in which multiple paths exist and the optimal path must be determined. In this case, the residual energies of candidate relay nodes are crucial to finding the optimal path when evaluating a number of alternate paths. Since only a single path has been analyzed here, this has not been considered.

Wang et al. present an extension of the work by Krogsveen et al. in [7]. Their optimization problem minimizes the total energy cost across a path as a function of the transmission rate and per-hop BER [7]. While the total energy across a path is minimized, the impact of resource consumption on energy capacity is not studied. This is crucial to meeting requirements for network lifetime in WSNs. Wang et al. also make an assumption of negligible interference that is critical to their problem, while a sensor topology or radio model is not presented. Thus, the study is theoretical.

Madan et al. proposes a cross-layer design based PHY, MAC and routing layers for TDMA-based WSNs [5]. The consideration of throughput is made to satisfy a maximum per-hop delay constraint, while the objective of the optimization is the maximization of the network lifetime. As has been discussed, next-generation WSNs will require joint considerations for both throughput and energy consumption in the optimization. By simply meeting a minimum throughput requirement, it is uncertain 
if we can achieve better performance. In other words, we may be able to identify sensors that are able to allocate resources more appropriately to throughput, with a better trade-off of throughput to energy consumption. This work also only considers string, linear and rhombus topologies, while residual energies of candidate routing nodes to determine real-time suitability are also not considered. This work also does not model the power consumption in the transceiver circuitry of transmitters and receivers, which is an important source of energy dissipation in wireless communications.

Another study by O'Neill et al. [41] studies rate and transmit power control in WSNs. This study derives a utility function to represent rate preferences, with constraints for the physical capacity of the link based on, for example, the maximum transmit power. A rate region is established for the utility function to identify the transmitter power allocation that is Pareto Optimal [41]. However, no constraints on energy consumption are provided and, thus, joint considerations for throughput and energy consumption are not made, while network lifetime is also not a consideration in this study. Similarly to [5], the study by O'Neill et al. does not study the energy efficiencies of multiple communication protocols and also does not consider energy dissipation of transceivers.

Wang and Sharif propose Distributed Source Coding (DSC) as an alternative to data aggregation in WSNs [42]. The DSC approach considers the correlation between data measurements at the decoder, as opposed to the encoder, such that packets with correlated measurements may be discarded. This is as opposed to data correlation 
that uses sensor resources to perform data aggregation prior to transmitting a single representative packet [42]. In [42], cross-layer optimization is performed for applications with correlated measurements such as real-time target tracking with joint rate and energy considerations. However, in their approach, sensors determine a transmission rate based on their residual energy level where each sensor will determine a next hop based on that rate. Sensors will coordinate to ensure that they only forward packets to nodes that have the same rate assignment [42]. While nodes should indeed control their energy consumption based on their residual energies, forcing nodes to choose their next hops by only considering neighboring nodes with similar rate assignments unnecessarily reduces the achievable end-to-end capacity of the transmission. A more intuitive solution may be necessary.

The Energy Optimization Approach (EOA) is proposed by Luo et al. in [43]. EOA considers joint optimal design of PHY, MAC and routing layers. Feedback transmission power control is performed at each node to determine the link suitability and, specifically, the minimum power necessary to reach each neighbor and to reduce interference. The routing layer selects the route with the least energy consumption to the sink, while this routing information is chosen to form scheduling schemes at the MAC layer. Decentralized optimization is used such that sensors coordinate to optimize the network. Other studies have also explored decentralized optimization to maximize network lifetime or minimize energy consumption [44][45][46][47][48]. While this is beneficial in theory, sensors may not have the sophistication necessary for decentralized coordination protocols given their limited resources [49]. 


\subsubsection{Cross-Layer Optimization in WSNs}

Other cross-layer studies include cross-layer route optimization for UWB systems by Chehri et al. in [1]. However, this paper only studies the PHY and MAC layer to minimize energy consumption in the network. Vuran and Akyildiz also propose a cross-layer design based on the PHY and MAC to exploit spatial correlation to reduce the amount of data routed in the network [50]. They propose correlation-based collaborative medium access control (CC-MAC) for event-based WSNs to enable single node transmissions to be representative of a number correlated sensor measurements.

Other studies such as LEACH [51] explore clustering algorithms to elect multiple sensors to cluster-head status. These cluster-head sensors perform data aggregation in addition to optimization functions such as scheduling on behalf of the sensor network. Their link suitability considers that a sensor's promotion to cluster-head status is dependent on received signal strengths such that minimal energy consumption in transmitting data is achieved. Sensors are elected on a rotating basis to distribute the energy consumption in the network. In practice, this may not be practical given the limited sensor resources in the network and a lack of sophistication that is necessary to govern a subsection of the sensor field. Throughput considerations are also not made in the derivation of link suitability for sensor network optimization. Other studies also explore clustering algorithms to elect multiple sensors to cluster-head status to perform data aggregation and optimization functions in the network [52][53][54][55][56]. Clustering algorithms will be further explored in Section 2.2.5. 
Specific research challenges have also been explored for particular applications. Akyildiz and Stuntebeck [57] studied the design challenges of deploying wireless underground sensor networks (WUSNs) specifically for below ground agricultural applications and environmental monitoring. They highlight topology challenges, power conservation, antenna design and the underground wireless channel. Lorincz et al. [58] study the inherent challenges facing sensor networks for emergency response systems. They point to device discovery as a critical challenge with devices being defined by the information that they can supply, i.e. one may want to request information from all sensor devices whose temperature is greater than $23^{\circ} \mathrm{C}$. Robust routing, prioritization of critical data, security and tracking are presented as other challenges facing next-generation WSNs [57]. For the biomedical field, Schwiebert et al. [59] present a number of other fascinating challenges for biomedical sensors including, but not restricted to, the material constraints as these sensors are implanted within the body and, thus, the material, size and shape is greatly restricted.

\subsubsection{Network Planning}

Network planning of energy capacities is studied in [60] to model the energy consumption of the processor for node control and data processing, transceiver for communication and sensing module of a wireless sensor. In [60], Zhou et al. also study multiple sensor states including off, transmit, receive, idle and sleep and aim to compare the energy consumption in these states. However, energy costs related to communication 
typically dominate a node's energy capacity over the energy consumption incurred by processing and sensing functions [8]. Also, the study does not consider throughput, coordination and data packets, and network lifetime in the modeling of sensor energy consumption. As a result, the study is missing critical constraints that affect both achievable throughput and energy capacity. The goal of our NPP is to model factors critical to network design that impact both performance and network lifetime objectives.

Another proposal by Chamam and Pierre in [61] performs energy planning to find the optimal allocation of states to sensors in WSNs that perform dynamic clustering. This is performed to maximize network lifetime under considerations of coverage, clustering and routing. Sensor states include on, off and cluster-head states since, as mentioned, clustering algorithms elect sensors to govern subsections of the sensor field on a rotating basis. To perform energy planning, Chamam and Pierre assume power consumptions across states and the initial sensor energies [61]. The study does not consist of modeling the required energy for sensors to manage both data and coordination packets, and variations in power consumptions across multiple communication technologies. Chamam and Pierre [61] also do not study multiple node types as they consider that sensors assume cluster-head duties on a periodic basis.

Another study by Gao studies energy consumption in ad-hoc WSNs and is interested in developing the relationship between energy consumption and non-protocols factors such as node density, network coverage and traffic density [62]. To do so, Gao defines the watt-per-meter metric as the average power required to move information 
per unit distance towards a destination node reliably. Throughput considerations are made for fixed data rates of $10 \mathrm{~kb} / \mathrm{s}$ to determine the bit-meter-per-joule metric. However, since only non-protocol factors are made in [62], Gao lacks consideration for various packet types for data and coordination, network lifetime and multiple node types such as sensors, cluster-heads and relay nodes.

A fourth proposal by Mergen et al. [63] studies energy and capacity considerations in modeling sensor networks with mobile access points. Optimization considerations are made for altitude, trajectory and coverage of access points, while capacities are studied in terms of short-term average power constraint. Mergen et al. do not model routing protocols or coordination packets to both provide feedback of node state information to access points and announcements of resource allocations backwards from the access point to the sensor.

\subsubsection{Routing in WSNs}

A significant amount of research has been done in the area of routing in WSNs. For typical sensor network scenarios, roughly $65 \%$ of traffic received at a sensor node is multi-hop traffic that needs to be forwarded to other nodes [64]. The routing protocols vary based on the inherent features of WSNs and the sensor network application requirements. Early protocols such as Sensor Protocols for Information via Negotiation (SPIN) [65][66] and directed diffusion [67] aimed to save sensor energy through negotiation and removal of redundant data in data-centric WSNs. However, 
each has their own limitations. While the amount of redundant data transmitted in the network is roughly halved using SPIN, the advertisement of a node's new data that is broadcasted to all of the node's neighbors requires a request for actual data delivery. This 3-stage protocol is required at each hop for end-to-end delivery. In large networks, this does not guarantee data delivery because it is likely that nodes between source and destination are not interested in the data and, thus, data might not reach the destination. As a result, for applications that need reliable data delivery such as ours, the SPIN protocol does not suffice [68].

In directed diffusion, an interest (query) is broadcasted by a BS and propagated through the network hop-by-hop, while an aggregation tree is set up in the reverse direction from the source toward the BS. Energy is saved as data aggregation is performed along the path to remove redundant data. Directed diffusion is not suitable for continuous data delivery (considering the overhead in setting up a path) or large networks where the propagation of the interest broadcast by ordinary sensors would waste significant energy resources. In our work, given the barrier in communicating between heterogeneous technologies of multiple clusters, such an approach is not feasible considering the number of messages that would require technology conversion through software radio. Thus, for our purposes, directed diffusion is also an impractical routing scheme.

Rumor routing [69] is another algorithm and is a variation of directed diffusion. In rumor routing, queries are forwarded to nodes that observe an event taking place. Thus, flooding of queries is prevented as only a small amount of data is requested. 
Rumor routing is an improvement over directed diffusion when the number of events is small [69], which is an assumption that may not be suitable for large sensor networks.

Several other routing approaches have been proposed including the Minimum Cost Forwarding Algorithm (MCFA) [70]. MCFA exploits the fact that the direction of routing is always known, i.e. sensors nodes are always aware of the direction of the fixed BS. Nodes broadcast their messages and each message is only forwarded if a neighbor receiving the message is on the least cost path to the BS [70]. Intermediatc relay nodes re-broadcast the message until it reaches the destination [70]. In sensor networks where sensors are generally dispersed randomly, it may not be realistic for sensors to determine the least cost path due to the coordination and sophistication required to gather this information. Also, along with other studies that only transmit packets to nodes that are en route to the BS, it is entirely possible that a neighbor of the source, which is slightly further away from the BS, may provide a more efficient path; these nodes may contain greater resources yet are ignored in the algorithm.

Routing approaches such as COUGAR [71] and ACQUIRE [72] use the sensor network as a distributed database system and use declarative and complex queries, respectively, to gather information from relevant sensors. However, they introduce a query layer into the protocol stack between the network and application layers. The associated overhead may lead to an undesirable drop in performance; rather than introducing additional layers, the focus should be on improving the efficiency of the protocol stack by either using cross-layer signaling or condensing layers to reduce delay. Surveys of other routing techniques in WSNs have been conducted in [68] [73]. 


\section{Energy-Aware Routing}

Energy-aware routing itself is a very broad field and deserves special attention when discussing routing in WSNs. One very interesting energy-aware routing protocol from Berkeley, known as Energy Aware Routing (EAR) of the same name, is proposed in [74]. In this study, EAR, which is a destination-initiated protocol, does not calculate a single optimal path between source and destination, but rather maintains a number of paths, each having a probability of being selected. The value of the probability depends on how low the energy consumption is on the path in relation to the others. Since different paths are chosen probabilistically, the residual energies of sensors along a single path will not deplete quickly. As a result, while it is similar to directed diffusion, energy-aware routing provides an overall improvement of $21.5 \%$ energy saving and a $44 \%$ increase in network lifetime over the directed diffusion protocol [74].

However, there are some drawbacks to the EAR protocol. Firstly, in nextgeneration networks in which application performance is a significant consideration, a path should not only be evaluated based on its energy consumption, but also as a function of the capacity achieved across the links. Secondly, there is no discussion of fairness in the multiple-source scenario, both in terms of source-contention (trade-off between a node forwarding its own data or relaying data for one of its neighbors) and destination-contention (queues at nodes closer to the destination filling up with packets from nearby nodes causing the packets of nodes far away to be dropped). Thirdly, in setting up a path, each intermediate node only forwards requests to its neighbors 
who are closer to the source node and farther away from the destination node than itself. While this may reduce the number of redundant messages in the network, a more intuitive approach may be achievable that is based on residual energies of neighbors. Fourthly, EAR requires that sensors have the sophistication necessary to perform distributed path calculations, which cannot be assumed in WSNs composed of simple and unsophisticated sensors.

Another approach to energy-aware routing from the National Institute of Information and Communications Technology in Japan studies route reconfiguration based on a decentralized optimization to maximize the network lifetime in WSNs using neural network dynamics [75]. It is clear that their decentralized method reduces the amount of transmitted data routed in larger sensor networks as coordination packets do not need to be sent to a centralized controller; however, it is again a matter of whether or not sensors can be relied upon for computationally intensive functions such as route computation. Their key assumption, which states that autonomous and decentralized computing algorithms are suitable based purely on the fact that sensor networks are composed of a large number of nodes, may not be realistic.

\section{Flow Augmentation Algorithm}

An interesting energy-aware routing algorithm known as the Flow Augmentation (FA) is presented in [76]. In this study, Chang and Tassiulas consider the residual energies of sensor nodes and the communication energy consumption rates in the network from source to destination [76]. These two considerations are reflected in the link costs, 
which are developed as functions of the residual energies and communication energy of the end nodes of the link. This is done in an effort to determine more suitable paths for WSNs that are energy-aware. Thus, since transmissions in WSNs occur on a packet-by-packet basis, it becomes possible to prevent the continual usage of the same routes en route to a master node, enabling the network to recover from node and link failures [76] [77]. It also enables us to maximize the overall lifetime of the sensors in the network. In utilizing the maximum lifetime routing path, traffic may switch to a different path even if there has not been a node or link failure. On the contrary, alternative paths are activated to distribute the computational load of routing traffic in the network whenever a new path is determined that has a higher residual energy than the working path [76] [77]. These paths can be disjoint or braided, and depends simply on the path with the lowest cost [78]. This is an improvement over EAR that select paths probabilistically to balance energy as the FA algorithm uses the path with the highest overall residual energy until a better path is found. Further details of the FA algorithm are presented here.

To determine the energy-aware routing path, Chang and Tassiulas consider that each sensor node $\imath$ in the network has initial battery energy $E_{\imath}$ and residual energy $\underline{E_{2}}$. It is also considered that the transmission energy that it takes node $\imath$ to transmit a unit of data to node $\jmath$ is $e_{\imath \jmath}^{t}$, while the energy consumed by node $\jmath$ in receiving that data unit is $e_{\imath \jmath}^{r}$. Let $\hat{Q}_{\imath}(T)$ represent the number of packets generated at node $\imath$ within the time interval $[0, T)$, while $\lambda$ denotes the amount of information routed between routing information updates. 
With this information, the FA algorithm is formed, which is used to calculate the energy-aware path with the best link cost function that will lead to the maximization of the system lifetime [76]. The first step of the algorithm calculates the shortest path from the source node to the destination; in terms of what we have discussed so far, the destination node is the master node. This shortest cost path is calculated using any existing shortest path algorithm such as the Dijkstra or Bellman-ford algorithms [76] where the path cost is computed as the summation of links costs on the path.

The link cost function used is,

$$
\operatorname{cost}_{\imath \jmath}= \begin{cases}\left(e_{\imath \jmath}^{t}\right)^{x_{1}}{\underline{E_{\imath}}}^{x_{2}} E_{\imath}^{x_{3}}+\left(e_{\imath \jmath}^{r}\right)^{x_{1}}{\underline{E_{\jmath}}}^{x_{2}} E_{\jmath}^{x_{3}} & , \text { if } \underline{E_{2}}-e_{\imath \jmath}^{t}>0 \\ \infty & , \text { otherwise }\end{cases}
$$

where $\left(x_{1}, x_{2}, x_{3}\right)$ are non-negative weighting factors for each item [76]. As shown above, in calculating the shortest path, the cost function is only utilized if sufficient energy exists on the link to carry the required traffic, i.e. $\underline{E_{\imath}}-e_{\imath \jmath}^{t}>0$; otherwise, cost $_{\imath \jmath}$ $=\infty$. By defining this cost function, they ensure that, as the residual energies of the end nodes of a link decrease, the corresponding cost of selecting that link increases. This allows the algorithm to avoid nodes with low residual energies in the routing process to maximize the overall network lifetime. As will be seen, the selection of $\left(x_{1}, x_{2}, x_{3}\right)$ allows us to determine if considering the communication energies will yield a longer network lifetime, and also whether it is better to consider absolute or relative residual energies in terms of the initial sensor energy $E$. 
The second step of the FA algorithm involves augmenting the residual energies as a function of an amount $\lambda Q_{\imath}$, where the use of the factor $\lambda$ scales the traffic generated at the node to represent the number of packets generated at the source node between routing information updates [76]. This allows the algorithm to adjust the residual energies of the end nodes of each link in terms of the amount of energy consumed to route traffic since the last update. The third step involves updating the residual energy information in the network. This is done just before, and mimics the process for, routing information updates [76] to generate new shortest-cost paths for the next iteration based on up-to-date knowledge of the node energies in the system. A summary of the FA algorithm $F A\left(x_{1}, x_{2}, x_{3}\right)$ is presented in Figure 2.1.

1. Calculate shortest cost path using shortest path algorithm with link costs $(i, j)$

2. If shortest cost path not found, then stop. Otherwise continue.

3. Augment links along shortest cost path by $\lambda Q_{i}$ by updating $\underline{E_{i}}$.

4. Perform residual energy update prior to routing information update.

5. Go to 1 .

Figure 2.1: The Flow Augmentation Algorithm [76]

\section{Building the Cost Function Through Feedback}

The $F A\left(x_{1}, x_{2}, x_{3}\right)$ algorithm allows us to provide comparisons of various methods through the selection of $\left(x_{1}, x_{2}, x_{3}\right)$ and their overall network lifetimes. For example, providing a comparison of $F A(1, x, x)$ and $F A(1, x, 0)$ allows us to compare absolute and relative residual energies in terms of overall network lifetime for our cost function. 


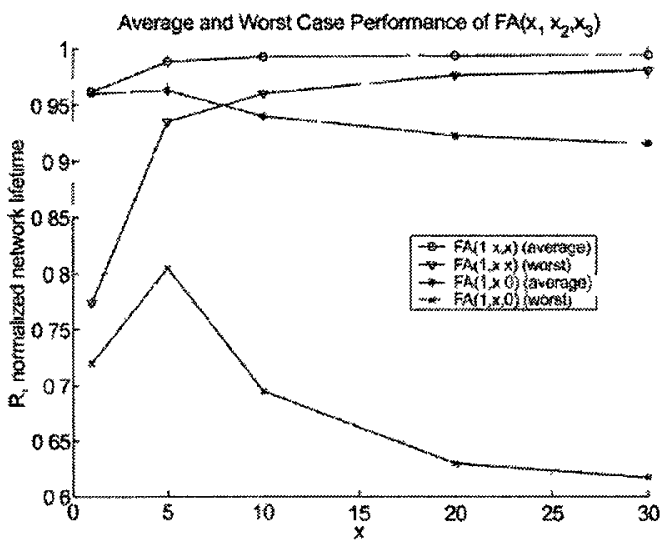

Figure 2.2: A Comparison of Absolute and Relative Residual Energies [76]

Secondly, a comparison of $F A(1, x, x)$ and $F A(0, x, x)$ allows us to determine whether or not the flow consumption energy should be considered in the link cost equation. Results for these two comparisons are provided in Figures 2.2 and 2.3, respectively.

As can be seen in comparing the average case performance in Figure 2.2, considering the relative residual energy in terms of the initial battery energy of the sensor, as shown in $F A(1, x, x)$, provides a normalized network lifetime that is superior to that of considering only the absolute residual energy in the $F A(1, x, 0)$ scheme. However, while improvement is only within roughly $7-8 \%$, the disparity between the two schemes is highly noticeable when considering the worst cases of the two schemes, where the $F A(1, x, x)$ is far superior. The improvement for both the average and worst cases increases as the weighting factor $x$ increases. Consideration of the flow consumption energy in Figure 2.3 shows that there is a large improvement in considering the flow consumption energy. This is shown in the roughly $70 \%$ improvement of the $F A(1, x, x)$ over the $F A(0, x, x)$ scheme. 


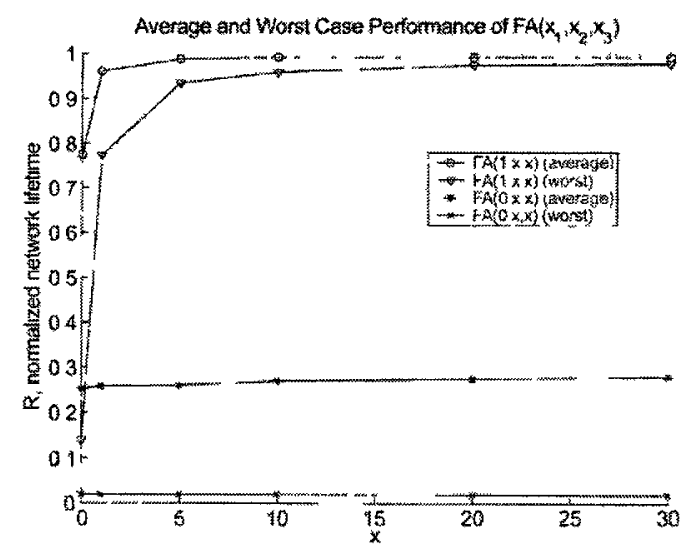

Figure 2.3: Consideration of Flow Consumption Energy [76]

Thus, as Chang and Tassiulas prove, in the design of a link cost equation for energy-aware routing, it is important to use the relative residual energy, as well as the flow consumption energy, in the link cost to maximize network lifetime. However, the flow consumption energy should be calculated from the actual allocation of physical resources to WSN transmissions, rather than simply from the traffic generated as in [76]. Otherwise, we cannot link energy dissipation to application performance to study the impact of physical resource consumption on energy capacity.

\subsubsection{Clustering}

Clustering is a concept for load balancing in wireless ad-hoc networks that has more recently been applied to WSNs. Typically in the discussion of hierarchical WSNs, sensor clustering is explored as a means to create multiple levels of responsibility among sensor nodes. This is typically performed by promoting sensor nodes dynamically to 
the role of cluster-head to balance the computational load throughout the network to create one or more clusters such as in [79][80][81][82][83]. In terms of routing, many of these studies additionally assume the use of a BS that is wired to a power source and, thus, only optimize the portion of a path to the cluster-head only. This is performed to achieve one of two main objectives: maximizing the network lifetime or minimizing energy consumption in routing information to a master node [84][85][86][87].

One such protocol is LEACH proposed by Heinzelman et al. in [51]. LEACH uses dynamic clustering and a randomized rotation of cluster-head duties with the goal of minimizing energy dissipation in the sensor network related to communication. Cluster-head roles include: creating a schedule for nodes in a sensor cluster; compressing and aggregating incoming data in transmitting to the BS; and, using high energy transmissions over far distances to reduce the number of nodes in the routing process. In their proposal, LEACH performs sensor to cluster-head optimization for minimum energy dissipation. The system determines the optimal number of clusters to have in the system and cluster-heads will self-elect themselves based on residual energy. The first assumption that complicates the sensor network problem is that each sensor can transmit to the BS; even though sensors only send data to their cluster-head in cluster-based models, cluster-heads are then responsible for communicating with the BS. Thus, sensors must be equipped with sufficient resources to handle these high energy transmissions if and when they are elected to cluster-head status. For large sensor networks, this may not be feasible while high energy transmissions may cause an exorbitant amount of interference. 
The Max-Min $d$-Cluster Algorithm is another clustering protocol proposed in [56]. In [56], Amis et al. aim to generate $d$-hop clusters to ensure that each node is only $d$ hops away from the cluster-head. While this is a favorable algorithm to reduce the number of hops used in routing, it does not ensure that the energy used in communicating to a master node is minimized and only studies the portion of the path to the cluster-head. Other protocols have been proposed in [54][87][88] that use clustering to consider cluster-sizes and cluster-head assignment. In [54], the optimal number of cluster-heads is explored in single level sensor networks to minimize energy use. In [87], clustering is used to study scalability and network lifetime in WSNs, while Chiasserini et al. propose Ad Hoc Network Design Algorithm (ANDA) to explore optimal cluster sizes and the optimal assignment of nodes to cluster-heads to maximize network lifetime [88].

It may actually be difficult to implement these sensor clustering algorithms in practice as they assume that sensors have both the sophistication and resources to perform cluster-head duties even if only on a rotating basis. Sensors are generally simple devices with limited hardware and energy capacity and, thus, expecting sensors to perform computationally intensive tasks may be unrealistic [89].

\section{Sensor Network Reconfiguration}

One interesting study of sensor network clustering by Chandrachood et al. presents several methods for sensor network reconfiguration [90]. Chandrachood et al. propose a multi-tier architecture that consists of a number of sensors that are distributed 
into one or more clusters, each governed by a cluster node. The network further introduces a master node that receives aggregated data from the cluster nodes in the network, as well as several other dormant master nodes that are dispersed throughout the field [90]. Sensor network reconfiguration considers that the energy levels of the cluster node that manages a subsection of the entire sensor field (synonymous to the cluster-head in our study) are depleted faster due to its more computationally intensive functions; however, as the job of the master node is even more computationally intensive than the cluster node, the sensor network is reconfigured in real-time to balance the computational load of the master node over the network. Hence, Chandrachood et al. aim to study the energy depletion at the master node for a number of these sensor network reconfiguration schemes.

One method of performing sensor network reconfiguration is by switching the master node functionality to redundant nodes in the field. Without doing so, if the master node fails, we run the risk of rendering the entire sensor field useless [91]. However, while we must consider that the energy levels of the master node deplete faster due to the computational intensity of their tasks, it is also important to consider the energies of the nodes surrounding the master node that are responsible for routing traffic to that master node. As a result, in the selection of one master node, not only are the energy levels of the master node important, but also its surrounding sensors [90]. This reduces time to node outages and network partitioning, as well as single points of failure, while allowing us to maintain the desired performance level of the network by increasing the overall life of the field. 
There are several methods for sensor network reconfiguration that make use of redundant master nodes (RMNs) that remain dormant until needed [90]. The first method consists of switching to nearby RMNs once the current master node has depleted. In this scheme, the designated master node will monitor its battery life along with that of other redundant master nodes in the field. At a specified level, say $5 \%$, the master node relegates its function to a nearby RMN [90]. While this will increase the lifetime of the network, it may not be a favorable scheme because nearby RMNs will typically receive traffic from the same surrounding sensors as the previous master node due to their proximity to one another [90]. As a result, this will further deplete the energy levels of surrounding sensors and may still quickly lead to network partitioning. Thus, this may not be the most survivable scheme.

An improvement to the previous scheme is to switch between RMNs in a predefined sequence prior to the depletion of the current master node. This reduces the battery drain of the surrounding nodes to the current master node by not over-utilizing those nodes to route traffic, thereby extending the life of the sensor field [90]. However, sequential switching provides a significant improvement only if the next master node that is selected is one that is not nearby to the current master node. If not, we run into the same issues as the previous scheme in over-burdening the same affected sensors used to route messages [90]. Typically, the master node switches its function to the next node in the sequence once its battery power drops to $75 \%, 50 \%$ and $25 \%$ of its initial level [90]. This helps to distribute the energy consumption among RMNs, as well as reduce the routing burden on surrounding sensors. 
The third sensor reconfiguration scheme involves switching master node functions to a RMN that has sufficient battery life remaining that is closest to a target being monitored. This scheme, although not applicable to all applications, reduces the drain on surrounding nodes because it reduces the burden from routing messages; those nodes taking readings of a target have shorter paths and fewer hops to route traffic to the new master node, increasing the overall life of the field. This is not a sequential scheme, but rather one that is dynamic and based on relative closeness to the target [90]. There are two disadvantages to the proximity-to-target scheme. Firstly, if the target being monitored moves rapidly it is possible that, upon switching to the RMN that is closest to the target, the same nodes are traversed in routing messages to the RMN. Secondly, selecting a master node close to a target may increase the probability of the sensor or its communication being detected [90].

Another scheme switches to the RMN that is furthest away from the current master node. This provides a better scheme than the others if the routing burden on the surrounding nodes of the current master node is reduced. Thus, this method aims to evenly distribute the computational load across all sensors in the network and attempts to lengthen the overall life of the sensor network [90].

The final method distributes the master node functionality between cluster nodes and rotates the role of master node between them. The selection of the next master node is also based on geometric location, i.e. the distance between the current master node and the new candidate is considered in the election of the new master node as in the previous scheme [90]. 


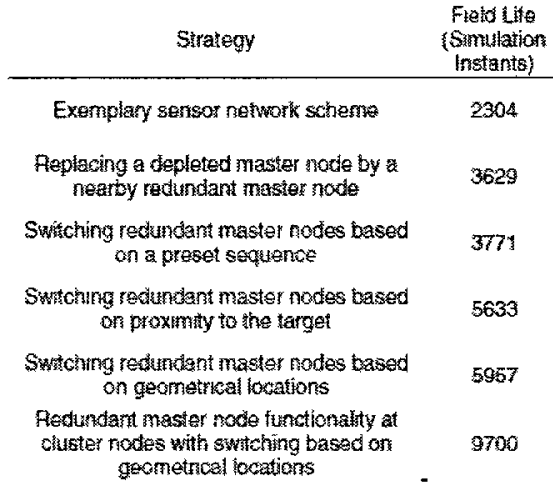

Figure 2.4: Field Life for the Exemplary and Reconfigured Sensor Networks [90]

The schemes discussed are compared in terms of overall network lifetime in Figure 2.4. As is shown, reconfiguration increases the lifetime of the network where master node functions of data fusion and controller communication are balanced within the network. The exemplary scheme is that which does not contain reconfiguration and, thus, has the lowest field life. Out of the next four schemes, reconfiguration based on geometric location provides the most favorable results. This is due to the fact that, by selecting the next master node by considering the proximity of candidates to the current master node, using the same intermediate relay nodes can be avoided. Thus, this balances the energy consumption due to routing and does not drain the same relay nodes excessively.

The scheme that provides the best results is the cluster node based approach for dual functionality as the cluster node and the master node, to further balance the master node responsibilities around the network. As stated, this scheme does switch master node functions based on geometric location within the set of cluster nodes. However, while the rotating master node function between cluster heads does improve 
the lifetime of the network, we cannot assume that it makes sense for all cluster nodes to be available for master node function. In our study, it is likely that one or more clusters are placed in a geographic location where fibre is not available; it would not make sense to set the mesh node that governs that cluster as the master node when mesh nodes that are more accessible to the processing center can be chosen. Thus, in our study, we only maintain a single master node (albeit with redundancy). However, we alleviate the concern of repeatedly using the same surrounding nodes in the network to route traffic to the master node by ensuring a high degree of connectivity in our mesh network.

\subsubsection{Preference Modeling and Utility Functions}

Preference modeling is used in microeconomic theory to evaluate a satisfaction gained by the consumption of goods and services. In network modeling, this satisfaction, or "utility", is commonly used to quantify the value of a resource, or combination of resources, to a node, which is typically modeled to act in its own self-interest, in a network. By placing these nodes in a market-based system, however, where goods are bought and sold, this inherent selfishness of nodes can be steered towards cooperative settings for the greater good of the network. Through the charging of prices for resource consumption, transmitters are encouraged to provide services while receivers do not hoard resources. This negotiation between transmitters and receivers is crucial for multi-hop systems where nodes have a choice between sending their own data or 
forwarding traffic for a neighbor node towards the master node. Since a node cannot be assumed to make this choice for the network's benefit instead of its own, incentives must be provided for the node to cooperate. Thus, assigning prices in market-based systems is extremely important in encouraging cooperation between transmitters and receivers in resource allocation algorithms.

One study that uses this approach [92] uses market-based systems for the design of a throughput allocation algorithm. In this paper, Wang and Li study marketbased interactions between pairs of nodes in service overlay networks that buy and sell units of throughput in file-sharing contexts. While [92] does not consider PHY layer resources, it is helpful in studying how transmitters and receivers, which act in their own self-interest, can be provided with incentives to share resources. This becomes a more interesting problem when studying the trade-off that relay nodes face, as stated, in either relaying traffic of another node or sending its own traffic instead.

\section{Utility Function for Resource Modeling}

The utility function is used in microeconomic theory to quantify the empirical benefit and empirical loss to a node of gaining or losing a unit of a resource [92]. In wireless systems, the empirica] benefit can be used to represent the satisfaction gained by a node in transmitting at a higher throughput, for example. Empirical loss, on the other hand, can be used to model the reluctance of the same node to diminish its power resource (or correspondingly the node's preference not to diminish its resource), 
knowing that the node has to give up some of its battery power to be able to transmit. As a result, this trade-off at each node defines the willingness of a node to participate in a transmission. By placing the proper prices on resource consumption and, as will be shown, this can be done through the assignment of scaling factors to the empirical benefit and loss functions, nodes within a network can be encouraged to cooperate.

In the work by Wang and $\mathrm{Li}$, the resource of interest is throughput. Each node's utility function specifies the revenue and cost, or correspondingly the value gained and lost, by selling and purchasing throughput resources within the market-based environment based on each node's service price $p(t)$. Since each node in [92] operates as both a transmitter and receiver, each node's utility function reflects both roles. The utility of a node $\imath$ at any time $t$ can be calculated as,

$$
\begin{aligned}
u_{\imath}(t)= & \epsilon_{1} \log \left(1+\frac{\sum_{\jmath \in U_{\imath}(t)} b_{\imath}^{\jmath}(t)}{C_{\imath}(t)}\right)+\epsilon_{2} \log \left(1-\frac{\sum_{k \in D_{\imath}(t)} b_{k}^{\imath}(t)}{C_{\imath}(t)}\right) \\
& -\sum_{\jmath \in U_{\imath}(t)} p_{\jmath}(t) b_{\imath}^{\jmath}(t)+p_{\imath}(t) \sum_{k \in D_{\imath}(t)} b_{k}^{\imath}(t)
\end{aligned}
$$

where node $\imath$ is assumed to have both incoming and outgoing capacities of $C_{\imath}$, transmits to a set of nodes $D_{\imath}(t)$ at rates $b_{k}^{2}(t), k \in D_{\imath}(t)$, and charges price $p_{\imath}(t)$ per unit of throughput. Similarly, $\imath$ receives throughput from a set of nodes $U_{\imath}(t)$ at rates $b_{\imath}^{\jmath}(t)$ and is charged price $p_{\jmath}(t), \jmath \in U_{\imath}(t)$. The two logarithms in (2.2) represent the empirical benefit of receiving flows and empirical loss of delivering flows for node $\imath$, respectively, while the last two terms represent the revenue and cost of buying and selling throughput, respectively, to node $\imath$ at time $t$. 
The empirical benefit $\epsilon_{1} \log \left(1+\frac{\sum_{\jmath \in U_{2}(t)} b_{z}^{3}(t)}{C_{\imath}(t)}\right)$ increases quickly from zero before it levels off, reflecting the intuition that the initial increase in receiving throughput is more important than further increases in consumption [92]. The empirical loss $\left|\epsilon_{2} \log \left(1-\frac{\sum_{k \in D_{2}(t)} b_{k}^{2}(t)}{C_{2}(t)}\right)\right|$, on the other hand, increases slowly from zero as the amount of its available throughput initially decreases, but the empirical loss increases sharply as its available bandwidth resource decreases towards zero. This accurately reflects the behavior of a selfish node in accepting the initial loss of its resource, but indicating an increasing reluctance to cooperate as its resource becomes more constrained [92]. The logarithmic function is ideal for modeling these preferences in optimization algorithms since they are monotonically increasing and strictly concave, making them quite useful when utilizing preference modeling strategies [92]. The coefficients $\epsilon_{1}$ and $\epsilon_{2}$ in (2.2) are positive weighting factors to scale the benefit earned and loss incurred in the overall utility.

\section{Throughput Optimization for Resource Allocation}

In a link negotiation between a prospective transmitter and receiver, it is the goal of the receiver in [92] to negotiate throughput with a set of candidate transmitters to maximize its utility function. Given that the application considered in [92] is file sharing, these transmitters are selected as those that have the file that the receiver is interested in downloading. The receiver queries the set of these transmitters $J_{i}(t)$ and determines the range of throughputs $\left(r_{m \imath n}^{j}, r_{m a x}^{j}\right), j \in J_{i}(t)$, that keeps its utility positive. This is required because it is possible that certain throughputs lead to a 
negative utility, especially at low service prices [92]. Upon determining the ranges $\left(r_{m i n}^{\jmath}, r_{m a x}^{\jmath}\right)$ for all candidate transmitters, the receiver forms the bounds of its objective function $\left(b_{\min }^{3}, b_{\max }^{3}\right)$, which it is going to maximize for each transmitter to find the candidate that offers the receiver its best trade-off of throughput $r_{2, D}^{3}$ to cost $p_{\jmath}(t)$. The objective function bounds $\left(b_{\min }^{\jmath}, b_{\max }^{\jmath}\right)$ are calculated as,

$$
\begin{aligned}
& b_{\min }^{\jmath}=r_{m i n}^{\jmath} \\
& b_{m a x}^{\jmath}=\min \left\{B_{\imath}(t), r_{\max }^{\jmath}\right\}
\end{aligned}
$$

where $B_{\imath}(t)$ represents the receiver's residual incoming capacity based on the amount of throughput the receiver is already receiving at time slot $t$ [92]. Upon finding its bounds $\left(b_{m i n}^{\jmath}, b_{m a x}^{\jmath}\right)$ for each candidate transmitter $\jmath$, the receiver maximizes its receiver utility function in (2.4) subject to these bounds to determine which candidate can give it the best rate $r_{\imath, D}^{J}[92]$.

$$
\begin{aligned}
& r_{\imath, D}^{\jmath}=\arg \max _{b_{\imath}^{\jmath}(t)} \epsilon_{1} \log \left(1+\frac{b_{\imath}^{\jmath}(t)+\sum_{\jmath^{\prime} \in U_{\imath}(t)} b_{\imath}^{\jmath^{\prime}}(t)}{C_{\imath}(t)}\right)-p_{\jmath}(t) b_{\imath}^{\jmath}(t) \\
& \text { s.t. } \quad b_{\min }^{\jmath} \leq b_{\imath}^{\jmath}(t) \leq b_{\max }^{\jmath}
\end{aligned}
$$

Upon determining which transmitter gives the receiver the best rate $r_{\imath, D}^{\jmath}$, the receiver informs the selected transmitter $j \in J_{\imath}(t)$ of its desire to begin the download, upon which $\jmath$ will update its own residual throughput $B_{\jmath}(t)$ based on the throughput selection $r_{2, D}^{3}$ 
Preference modeling allows us to perform a resource negotiation between nodes in wireless networks that have different preferences, resource availabilities and operate under standard requirements of different wireless technologies. In this fashion, we can provide incentives for these transmitters and receivers to cooperate, not hoard resources and, most importantly, not waste already constrained sensor resources such as power by setting our prices appropriately to prevent excessive consumption.

\subsection{PHY Layer Resources and FDMA Capacity}

As we are using the FDMA capacity to allocate UWB, 802.16-2004 Worldwide Interoperability for Microwave Access (WiMax) and Zigbee transmissions over different channels over the spectrum band, it is important to review the achievable FDMA capacity via the Shannon capacity. This allows us to relate the PHY layer resources $r$ on channel $f$ to the achievable capacity and enable us to perform adaptive allocations of resources to transmissions in our heterogeneous WSN.

The Shannon capacity for FDMA can be found in [93]. In the FDMA model, a transmitter $n$ sends information to receivers in a broadcast fashion over a set of outgoing Gaussian channels $F$. The channels $f \in F$ for a given technology used in these transmissions are disjoint with bandwidths $W_{f} \geq 0$ and received signal powers $P_{f} \geq 0$. The receivers of these transmissions are subject to independent additive white Gaussian noise (AWGN) with power spectral densities $\sigma_{f}$. The theoretical capacity $c_{f}=\gamma(r)$ of an FDMA Gaussian channel is shown in (2.5) as a function of 
the PHY layer resources $r=\left(P_{f}, W_{f}\right)$ and channel interference $I_{f}$,

$$
c_{f}=\gamma\left(P_{f}, W_{f}\right)=W_{f} \log _{2}\left(1+\frac{P_{f}}{\sigma_{f} W_{f}+I_{f}}\right)
$$

The classical Shannon capacity formula is concave and monotonically increasing [93]. These theoretical rates cannot be achieved entirely in practise. This is due to regulatory limits and interference caused by the allocation of communication resources to other links in the network that reduce the achievable capacity [93].

\subsection{Modeling Energy Efficiency}

The power consumption of the radio in embedded devices considers both the transceiver and start-up power consumption in utilizing the radio [2]. We may compare the power consumption between radios of multiple wireless technologies by calculating the energy efficiency of a technology, $q_{t}[94]$. The energy efficiency, $q_{t}$, is modeled as the energy consumption per payload bit processed by the radio of the embedded device and is defined as,

$$
q_{t}=q_{t x}+q_{r x}+\frac{E_{d e c}}{l}
$$

where $l$ is the payload length of a packet used by a communication protocol and $E_{d e c}$ is the energy required to decode a packet. The quantities $q_{t x}$ and $q_{r x}$ are the energy consumptions per payload bit of the radio in transmitting and receiving, respectively, 
and are given by,

$$
q_{t x}=\frac{\left(\left(P_{t e}+P_{o}\right) \frac{(l+\alpha+\tau)}{R}+P_{t s t} T_{t s t}\right)}{l}
$$

and,

$$
q_{r x}=\frac{\left(P_{r e} \frac{(l+\alpha+\tau)}{R}+P_{r s t} T_{r s t}\right)}{l}
$$

where $P_{t e / r e}$ are the powers consumed in the transceiver circuitry of the radio, $P_{t s t / r s t}$ are the start-up powers of the radio and $T_{t s t / r s t}$ are the start-up times for transmitting/receiving [94]. $P_{o}$ is the transmit power, and $\alpha$ and $\tau$ are the header and trailer bit lengths of the packet [94]. The energy consumptions per payload bit for various technologies were provided in Tablc 1.2 .

The modeling of the energy efficiency of a radio enables us to determine the power consumption due to a transmission, in separating the portions of the power consumption due to transmitting and receiving. As the energy efficiency, $q_{t}$, considers the power consumption in the radio, it should not be confused with the achievable $E_{b} / N_{0}$ of the communication channel itself.

\subsubsection{Power Consumption due to a Transmission}

For modeling the power dissipation due to communication, we consider the transmit power $P_{o}$ selected for a transmission, the transceiver load $P_{t e / r e}$ due to transmitting at a certain rate $R$, and the duration of the transmission. By manipulating (2.7) and 
(2.8), we can consider the full packet size $m$ to determine the energy consumption per packet bit and yield,

$$
\frac{q_{t x} l}{m}=\frac{\left(\left(P_{t e}+P_{o}\right) \frac{m}{R}+P_{t s t} T_{t s t}\right)}{m}
$$

and,

$$
\frac{q_{r x} l}{m}=\frac{\left(P_{r e} \frac{m}{R}+P_{r s t} T_{r s t}\right)}{m}
$$

Having derived the energy consumptions per packet bit, the energy dissipation for a transmitter $v_{x}$ is,

$$
E_{v_{x}, d \imath s s}=\left(\frac{q_{t x} l R}{m}\right)\left(\frac{m n}{R}\right)=q_{t x} l n=\zeta q_{t_{v_{x}}} l n
$$

as the product of power consumption and the duration of the transmission, and includes $P_{o}, P_{t e / r e}, P_{t s t / r s t}$ and $T_{t s t / r s t}$. In (2.10), $\zeta$ represents the fraction of the energy consumption for a transmission allocated to a transmitter itself. For the receiver $v_{y}$ the energy dissipation due to a transmission can be calculated in a similar fashion as,

$$
E_{v_{y}, d r s s}=\left(\frac{q_{r x} l R}{m}\right)\left(\frac{m n}{R}\right)=q_{r x} l n=(1-\zeta) q_{t_{x}} l n
$$

The energy consumed by listening for a transmission, receiving and transitioning to receive mode may be similar to the energy consumed by transmitting [95]. As a result, we choose $\zeta$ to split the energy drain evenly between $E_{v_{x}, d r s s}$ and $E_{v_{y}, d r s s}$ according to [95]. It is known that, in a transmission, the power consumed by a transmitter is 
significantly larger than that of a receiver; however, considering the energy dissipated by a receiver in constantly listening on a channel prior to the transmission, the cost of listening may be the dominant factor in overall energy consumption [63]. In reality, the energy split between a transmitter and receiver considering the various transceiver states depends on the communication protocol and the transceiver design. These determine the split between the transmitter and receiver energy consumption for a transmission. Upon determining $E_{v_{x}, \text { drss }}$ and $E_{v_{y}, d r s s}$, the energy drain is deducted from the remaining energy capacities of the transmitter and receiver to update the state of the network.

\subsection{Critique of Literature}

The next-generation of WSNs will play a significant role in the future of maintenance and control applications. A review of previous research on the state-of-the-art in WSNs shows several assumptions and design choices that are not suitable to such an architecture. For example, many of the studies presented either do not perform joint throughput and energy optimization, do not model these two optimization factors in terms of physical resources that affect both throughput and energy capacity, or do not perform cross-layer design of the PHY, MAC and routing layers. As a result, they are missing a major piece in the cross-layer optimization of application-centric WSNs. Other studies do not consider communication protocol design in modeling the energy consumption due to routing. Protocol design is important given the use of multiple 
technologies in our network. Meanwhile, those that use clustering algorithms and decentralized optimization assume that sensors have the sophistication to manage and optimize subsections of the sensor field, which may not be realistic. A network only composed of sensors is also not suitable for large multi-hop networks given the short range characteristics of sensor communication protocols; the depletion of energy resources due to multi-hop transmissions would be significant.

As a result, we propose the distributed WSN architecture that uses a mesh network as an enabling technology. The mesh network expands the reach of the network and performs optimization with greater resources on behalf of subordinate sensors. Our aim is to design cross-layer optimization protocols for distributed WSNs that directly tie physical resource consumption to achievable throughput and the corresponding energy consumption. In this fashion, we are able to achieve significant throughput performance, while maintaining reasonable network lifetimes. Also, by using clusterheads to perform optimization, sensors can focus on sensing and data transmission, enabling us to allocate sensor energy to improve transmission capacity.

\subsection{Summary}

This section provided the necessary background for developing our distributed WSN problem. This included: the performance limits of WSNs; the state-of-the-art in throughput and energy optimization, cross-layer optimization and network planning 
of these networks; the relationship between the FDMA capacity and PHY layer parameters; preference modeling for resource allocation algorithms using utility functions; and, the definition of the energy efficiency of a communication protocol. The next chapter will provide our problem formulation and the wireless models used in this study. 


\section{Chapter 3}

\section{Problem Formulation}

\subsection{Introduction}

This research explores throughput and energy optimization in distributed and application-centric WSNs. The goal of our study is to achieve significant throughput performance, while performing energy conservation such that our network meets a desired network lifetime. We aim to derive a solution that is independent of the selection of any particular communication protocol.

To perform this study, we design a distributed system that uses two sensor clusters, one based on 802.15.3 UWB and the other using 802.15.4 Zigbee technology. These communication protocols are used to test our cross-layer optimization policy. These clusters are connected using an 802.16-2004 WiMax mesh network that acts as a high capacity backbone in transferring gathered data from sensors to a master node. WiMax communication, as opposed to $\mathrm{WiFi}$, is preferred to reduce the density of the mesh network and the number of mesh nodes to provide adequate coverage in 
the network. WiFi may be used as a substitute but requires a denser mesh network with a larger number of nodes to compensate for the reduction in transmission range than is provided by WiMax. With state-of-the-art technologies, among others that will emerge in the future, our solution is applicable to any network seeking joint throughput and energy optimization in application-centric WSNs.

In this dissertation, a cross-layer optimization policy is designed for applicationcentric WSNs to assign paths, operating channels and transmit power to FDMA allocations. We use these resources to identify the optimal multi-hop path for a source sensor in routing sensory data to the master node based on cross-layer considerations. The optimal path is that which has the highest trade-off between throughput benefits and energy costs across multiple transmission channels.

We place our receivers and transmitters in a system that mimics the market used in [92]. However, in our study, nodes are not placed in a market-based environment where transmitters charge prices for the consumption of resources, but rather assign costs to utilize their links in the chosen multi-hop path subject to their residual energy levels. By charging appropriate prices as link costs that are a function of resource availability, we can force receivers to consider the limited resources of transmitters in the network on a hop-by-hop basis; in this manner, our solution avoid paths with links that have lower resource availabilities. This is similar to the effect of charging prices in [92] to force nodes, which generally hoard resources, to cooperate.

The basis of the interaction between a transmitter and receiver is the negotiation of resources for the optimal channel and power allocation for a single hop. This is 
calculated at the cluster-head in a centralized fashion to evaluate the suitability of the link in question for a candidate multi-hop path. By exploiting the relationship of bandwidth, signal power and operating frequency to the FDMA capacity, we design a robust and intelligent spectrum assignment for this multi-hop path. Transmitters and receivers negotiate to tune the operating frequency to more spectrum friendly channels to avoid interference. In addition, transmit power is selected based on the current state of interference in the network. Throughput is also be optimized by considering the power drain the data rate would cause based on the communication protocol being used.

This chapter will provide a thorough formulation of our cross-layer optimization problem, our formal problem definition and the assumptions made in conducting our research. We will also derive the objective function used in selecting the optimal multi-hop path, operating frequency and signal power in the cross-layer optimization policy. Our model to implement energy consumption and the wireless system models used in our study for UWB, Zigbee and WiMax will also be presented.

\subsection{Network Architecture}

Our multi-tier network architecture is presented in Figure 3.1. Mesh nodes are organized in a hexagonal topology for maximum connectivity. These mesh nodes are placed at the center of their clusters around which UWB and Zigbee sensors are positioned randomly. 


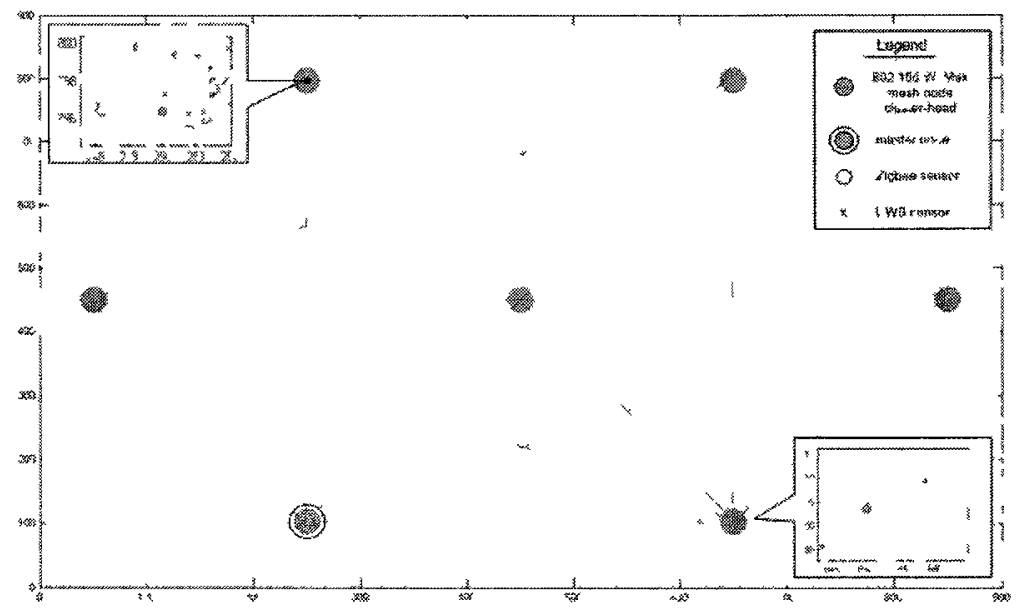

Figure 3.1: The Distributed WSN Architecture

While we would ideally like to maintain a hexagonal topology of mesh nodes in practice, this may not always be achievable. The organization of mesh nodes depends highly on the sensory locations of interest. For example, if sensor clusters are deployed to monitor the infrastructure of one or more bridges or buildings, or the military is interested in monitoring specific high security areas, it may not always be possible to deploy a mesh node in an ideal location. Thus, the mesh topology depends strictly on the locations at which these sensor clusters need to be placed. Thus, we present the topology in Figure 3.1 as the default mesh topology to study the throughput and energy problem. It is important to note that, in networks for which a hexagonal mesh topology is not possible, network planners should be aware of potential single points of failure. In these cases, load balancing or redundancy should be explored to ensure that mesh nodes are not overburdened with routing responsibility 


\subsection{Problem Definition}

Define our multi-tier sensor network $N=\left(G\left(C_{l}\left(V_{\imath}, M_{\imath}\right), E_{l}\right), S\right)$ that is composed of a set of sensor nodes $V_{\imath}$ and mesh nodes $M_{\imath}$. These sensor and mesh nodes are organized into clusters $C_{l}\left(V_{\imath}, M_{\imath}\right)$ and connected in a two-level network via directed link set $E_{l}$. The nodes are connected according to a topology $G\left(C_{l}\left(V_{\imath}, M_{\imath}\right), E_{l}\right)$ that is presented in space $S$ in Figure 3.1. We make the following additional definitions:

- the set of all nodes in the network, $Z=V_{\imath} \cup M_{i}$;

- the set of technologies available to each node in the network, $T=\left\{t_{v_{x}} \mid \forall v_{x} \in Z\right\}$;

- the number of sub-networks (sensor clusters and mesh network) in the distributed WSN, $S_{N}$

- the expected number of neighbors of a sensor node in a cluster, $E\left[N_{s}\right]$;

- the expected number of neighbors of a mesh node in the mesh network, $E\left[N_{m}\right]$;

- the set of residual energies of all nodes in network, $E_{a v a n l}=\left\{e_{v_{x}} \mid \forall v_{x} \in Z\right\}$;

- the set of initial energies of all nodes in network, $E_{\imath n i t}=\left\{E_{v_{x}} \mid \forall v_{x} \in Z\right\}$;

- the set of channels available to all nodes of a given sub-network, $F_{S_{N}}=$ $\left\{f_{t_{v_{x}}} \mid \forall t_{v_{x}} \in T\right\}$

- the energy efficiencies of various communication protocols, $Q=\left\{q_{t_{v_{x}}} \mid \forall t_{v_{x}} \in T\right\}$;

- the master node, $v_{3} \in M_{i}$; 
- a cluster-head node, $v_{m} \in M_{i}$;

- the maximum number of hops in a path, $K^{\prime}=4$;

- the path between any sensor $v_{\imath}$ and master node $v_{\jmath}$ through cluster-head $v_{m}$, path $_{v_{2}, v_{\jmath}}=\left\{v_{2}, \ldots, v_{m}, \ldots, v_{\jmath}\right\}$, where the number of nodes along a simple path between $v_{\imath}$ and $v_{\jmath}$ can be computed as, $K=n\left(\right.$ path $\left._{v_{\imath}, v_{\jmath}}\right) \leq\left(K^{\prime}+1\right)$; and,

- the $\left(K^{\prime}+1\right) \times W \times\left|V_{\imath}\right|$ matrix, $X$, of all the paths from all sensor nodes in the network to the master node, where $W$ is the maximum number of paths over all nodes. $\left|V_{\imath}\right|$ is the cardinality of the sensor node set.

Communication protocols have varying dependencies on multi-hop routing given their variations in acceptable transmit powers and maximum transmission ranges. As such, they will likely have different path lengths. However, energy consumption characteristics between a transmitter and receiver suggest that it is better for the network to reduce the number of hops in a path at the expense of using a higher transmit power [2]. As a result, we have set a limit of $K^{\prime}=4$ as the maximum number of hops along a path to restrict the number of nodes used to route traffic. Based on our network topology, there must be at least one-hop in both the sensor and mesh networks given our two-part path; as a result, our selection of $K^{\prime}$ leaves sufficient hops for a sensor to route traffic to the cluster-head given the density of our sensor network. Reducing it further may adversely affect routing in the UWB cluster, for which the transmission range is only 10 meters. We shall derive the expected number of simple paths, $E[W]$, from a single source sensor to the master node in Chapter 4 . 


\subsection{Optimization Parameters of Interest}

The cross-layer optimization policy designed in this work focuses on functions at three layers: routing, or path selection, at the network layer; channel selection at the link layer; and, transmit power allocation at the PHY layer:

- Transmit power and throughput: To compute the optimized link suitability, it is necessary to find the optimal trade-off between throughput and the corresponding energy consumption in terms of the PHY layer resource of transmit power. We do so by modeling the preferences of nodes with utility functions both in terms of the achievable throughput benefit and the desire of the node to incur a higher power cost. We also model the transceiver power consumption. To ensure a valid transmit power allocation, we restrict the range of optimization with resource constraints. The selection of transmit power and throughput for a single channel leads to the optimized link suitability;

- Channel selection: At the link layer, we consider the optimal channel allocation for a link in a candidate path based on a preference ranking of link utilities from the PHY layer over all channels. With frequency-agile wireless sensors, it is possible to select optimal frequencies at each hop based on the current interference across the spectrum band. By allowing the receiver in a resource negotiation to rank its most preferred channels (in order of highest to lowest link utility), our policy finds the links with the highest overall preference ranking for the channel allocation; and, 
- Path selection: At the network layer, we are interested in finding the path with the largest trade-off of throughput to energy consumption in routing traffic to the master node. The paths under consideration are simple, in that they do not contain multiple instances of a single node and, thus, routing loops. A path also exists in two parts, within the source sensor's own cluster and in the mesh network. The selection of the optimal path is a three-step cross-layer process: enumerating over all candidate paths with a maximum of $K^{\prime}$ hops between the source sensor and the master node; optimizing the link utilities at the PHY layer and the channel assignment at the link layer for each hop along the candidate paths; and, selecting the candidate path that has the maximum average profit across all links. This average represents the suitability of using a candidate path in the routing process.

This chapter will discuss the optimization of link utilities and our objective function. Further details regarding the cross-layer design and the functions of individual layers such as channel scheduling are the focus of Chapter 4.

\subsection{Assumptions}

To perform this study, we make the following assumptions in the design of a crosslayer optimization policy for distributed WSNs using overlay mesh networks:

1. All clusters perform periodic data delivery according to a Poisson process with exponentially distributed inter-arrival times of events with parameter $\lambda$; 
2. Sensor nodes are frequency-agile and can tune their frequencies to select different operating channels. Many papers have studied frequency-agile WSNs from the perspective of bandwidth allocation including [36] [96];

3. Mesh nodes in next-generation WSNs use software radio to communicate with both sensor nodes and mesh nodes over heterogeneous wireless technologies. Software radio is required to tune operating parameters from one technology to another. This is a necessary assumption because bridges between sensors and mesh technologies are currently only available in the market for Zigbee. While software radio for UWB remains unavailable, this study may use Zigbee bridges to deploy distributed WSNs of Zigbee sensor clusters, although for a limited set of applications. Note that the energy required to convert transmissions from one set of technological parameters to another is not yet considered in our study;

4. Each cluster-head is equipped with two wireless NIC cards: one to communicate with sensors in the cluster, and the other to communicate with its mesh peers, over different communication protocols. This is the necessary assumption for software radio to ensure that the mesh node may communicate with both sensors and mesh devices, and does not interfere with the formation of the network;

5. All nodes within a cluster use omni-directional antennas with equal gains;

6. Neither sensor nodes nor mesh nodes are wired to power sources. This allows us to explore a general architecture and expands the number of environments 
in which, and applications for which, the system can be deployed. In reality, certain mesh nodes may be connected to power sources if the locations in which the mesh nodes are deployed have power sources readily available. Following the approach presented in this thesis, these mesh nodes with unlimited power availabilities would be favored in the routing process over mesh nodes with limited power resources;

7. Mesh nodes are placed in a hexagonal topology for topology optimality and maximum network coverage [97] [98]. In practice, a hexagonal topology may not be practical and mesh nodes will be placed at sensory locations of interest. However, we use the hexagonal topology here to design a general two-tier architecture;

8. Sensor nodes are aware of their positions and are equipped with Global Positioning System (GPS). This assumption allows us to focus on the throughput and energy optimization problem. The sensor localization problem will be considered in future work;

9. The master node is a mesh node and the sink in the network. The master node may be placed at a processing center or at a desirable location with which to communicate with the processing center;

10. Each sensor node follows a $M / M / 3 / 3$ queueing model with three operating channels and no queue. This assumption is made to limit the competition 
between all nodes to only three channels to leave sufficient capacity available to carry relay traffic. Single channels are not used end-to-end for a path and each hop chooses a different operating channel;

11. Sensor nodes in both of our clusters perform sensing functions with the same frequency and have equal power drain due to these sensing functions; and,

12. The cluster-head has real-time channel state information, which can be assumed because our sensor nodes are fixed, allowing us to assume a slow fading model. In the slow fading model, the coherence time of a channel is greater than the symbol time $\left(C_{T} \leq C_{S}\right)$. This ensures that the channel remains constant long enough to allow feedback of new channel conditions to the cluster-head for the optimization process.

\subsection{Link Utility Function}

Define the communication resources $r_{f}$ associated with a wireless channel $f \in F$, where $F$ is the channel set of a given communication protocol. The capacity of a channel $c_{f}$ is dependent on a number of factors including $r_{f}$, but in this study we focus on the case where $c_{f}=\phi\left(r_{f}\right)$ only. The transmission rate $R_{v_{x}, v_{y}, f} \leq c_{f}$ is defined as a fraction of the FDMA Shannon capacity for $r_{f}=\left(P_{v_{x}, f}^{t}\right)$ as,

$$
R_{v_{x}, v_{y}, f}=\phi\left(P_{v_{x}, f}^{t}\right)=w_{f} \log _{2}\left(1+\frac{P_{v_{x}, f}^{t}\left|H_{v_{x}, v_{y}, f}\right|^{2}}{w_{f} N_{v_{y}, f}+I_{v_{y}, f}}\right)
$$


where our resource of interest $r_{f}=\left(P_{v_{x}, f}^{t}\right)$ is the selected power at transmitter $v_{x}$ in sending data to receiver $v_{y}$ on wireless channel $f,\left|H_{v_{x}, v_{y}, f}\right|^{2}$ is the channel gain between $v_{x}$ and $v_{y}, N_{v_{y}, f}$ is the Gaussian noise power on the channel from the perspective of the receiver $v_{y}$, and $I_{v_{y}, f}$ is the interference of $v_{y}$ on channel $f$. As we form our link utility function, it shall evaluate the allocation of our resources $r_{f}=\left(P_{v_{x}, f}^{t}\right)$ for link $v_{x}-v_{y}$ in terms of the achievable transmission rate $R_{v_{x}, v_{y}, f}$ and the power cost associated with transmitting at rate $R_{v_{x}, v_{y}, f}$ over the link.

The Shannon capacity may be used to model the maximum error-free information data rate that is achievable for a given communication protocol on a transmission link [99]. As such, the use of the Shannon capacity enables us to remove the dependency of our cross-layer optimization policy on any single communication technology. It also allows us to perform adaptive resource allocations based on the real-time state of the network by relating the data rate to PHY layer parameters. In our study, $r_{f}=$ $\left(P_{v_{x}, f}^{t}\right)$ only because we consider that all channels have equal bandwidths $w_{f}$. Since the Shannon capacity is theoretical, we limit the Shannon capacity with additional regulatory limits.

Our link utility function $L_{v_{x}, v_{y}, f}\left(R_{v_{x}, v_{y}, f}, t\right)$ is the basis of the resource negotiation between a prospective transmitter and receiver in the network. It quantifies the link suitability and is shown below,

$$
L_{v_{x}, v_{y}, f}\left(R_{v_{x}, v_{y}, f}, t\right)=\alpha \log _{10}\left(1+\frac{R_{v_{x}, v_{y}, f}}{C_{v_{y}, f}(t)}\right)-\psi(E) \frac{q_{t_{x}} l R_{v_{x}, v_{y}, f}}{m}+\eta
$$




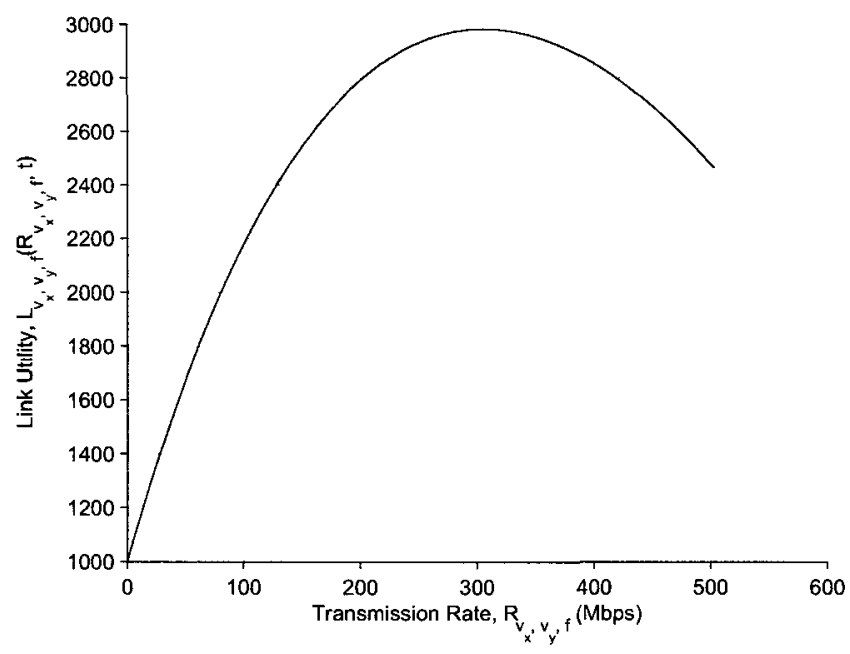

Figure 3.2: Example of a Link Utility Function $L_{v_{x}, v_{y}, f}\left(R_{v_{x}, v_{y}, f}, t\right)$

The link utility is formed from the receiver's perspective as the difference between a benefit function and a cost function to determine the overall profit gained by using a link. Figure 3.2 shows a sample plot of the link utility function $L_{v_{x}, v_{y}, f}\left(R_{v_{x}, v_{y}, f}, t\right)$, which is concave as a function of the data rate $R_{v_{x}, v_{y}, f}$.

The first term of $L_{v_{x}, v_{y}, f}\left(R_{v_{x}, v_{y}, f}, t\right)$ in (3.2) represents the rate benefit, i.e. the benefit gained as a function of the transmission rate $R_{v_{x}, v_{y}, f}$. The rate benefit is modeled by a logarithmic function which is monotonically increasing and follows the law of diminishing returns. As a result, an initial increase in $R_{v_{x}, v_{y}, f}$ is more important to a node than further increases in $R_{v_{x}, v_{y}, f}$, as the node approaches the incoming capacity of the channel, $C_{v_{y}, f}(t)$. The benefit function that comprises the link utility in Figure 3.2 is shown separately in Figure 3.3(a). The appearance of the benefit function will be discussed shortly. 


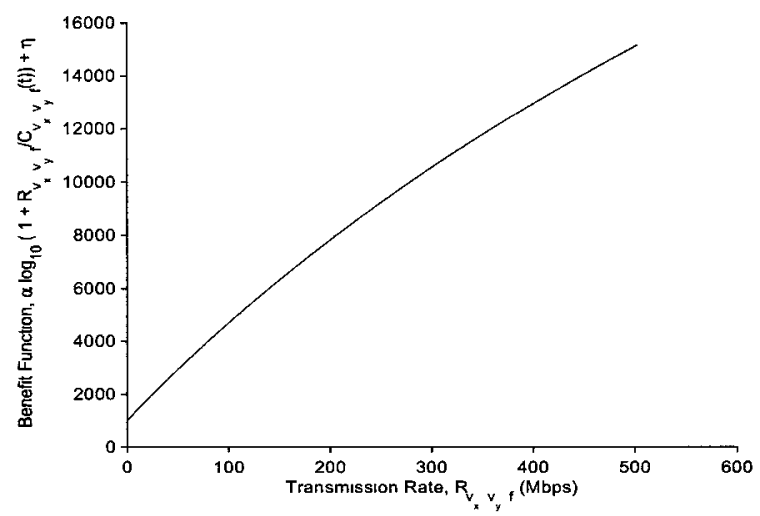

(a) Benefit Function

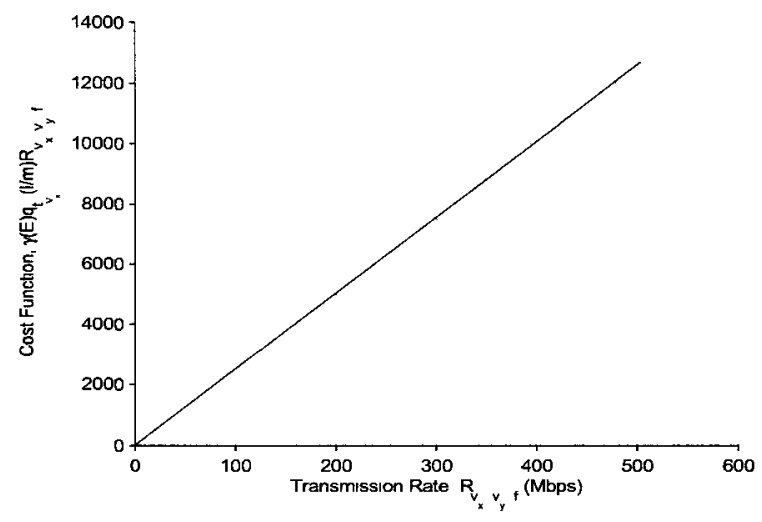

(b) Cost Function

Figure 3.3: Examples of Benefit and Cost Functions to Form Link Utility

The cost function is shown in the second term of (3.2). The cost function models the linear power cost of utilizing a link in the optimal path. The power cost is a function of the achievable transmission rate $R_{v_{x}, v_{y}, f}$ and the energy efficiency (including transceiver power consumption) of the technology used for the communication link, $q_{t_{v_{x}}} \in Q$, as defined in Chapter 2. By multiplying the energy consumption per payload bit, $q_{t_{v_{x}}}$ (in joules $/$ bit), by the ratio of $l / m$, we retrieve the energy efficiency of 
the technology in considering the full packet size including overhead bits. The power consumption in sending a packet over the prospective link (in watts) is found by multiplying the energy consumption per packet bit (in joules/bıt) by the transmission rate $R_{v_{x}, v_{y}, f}$ (in bits/sec). The result is the amount of energy over time, or power, used in transmitting a packet over the link. This power cost is further scaled by a function $\psi(E)$ of the transmitter and receiver's residual energy $E$. The power cost function that contributes to the link utility $L_{v_{x}, v_{y}, f}$ in Figure 3.2 is also shown separately in Figure 3.3(b). The formation of the cost function is critical to our study - it ensures that the power cost incurred by utilizing a link is a function of the achievable transmission rate $R_{v_{x}, v_{y}, f}$. By using this approach to model out power cost, we ensure that optimizing the link suitability yields the optimal trade-off between throughput and energy consumption in allocating PHY layer resources to a transmission.

In the derivation of the power cost, we consider the residual energies of the transmitter $v_{x}$ and receiver $v_{y}$ in the formation of $\psi(E) . E$ is maximum of the inverse residual energy of the transmitter $v_{x}$ and receiver $v_{y}$ based on real-time energies as proposed by Chang and Tassiulas in [76],

$$
E=\max \left(\frac{E_{v_{x}}(t)}{e_{v_{x}}(t)}, \frac{E_{v_{y}}(t)}{e_{v_{y}}(t)}\right)
$$

By including the residual energy of the transmitter and receiver in the link suitability, if the residual energy of either the transmitter or receiver is low, the cost of utilizing a link in the path is high, thereby decreasing the profit gained by utilizing 
the path. Similarly, if the residual energies of the transmitter and receiver are high, the cost of utilizing the link is low; this has the effect of increasing the profit. As a result, we can avoid relay nodes with lower residual energies in the optimization process.

The need for $\psi(E)$ arises from the fact that the range of available transmit power is extremely small, on the order of microwatts to milliwatts. As a result, the algorithm is sensitive to small changes in cost. Consider a situation where a node has half of its battery power remaining; while it should be able to operate normally, simply using $E$ in the power cost, given our example, essentially doubles our link cost. Thus, in the absence of a function like $\psi(E)$, the algorithm may force the selection of zero transmit power. The effect is worse at larger $R$, due to a significantly higher link cost. As a result, $\psi(E)$ flattens out the power cost as a function of the residual energy $E$ to ensure that the algorithm operates successfully within a very small optimality range.

The function $\psi(x)$ is of the form,

$$
\psi(x)=\beta \ln (x)+\gamma
$$

for a UWB and WiMax transmission, and of the form,

$$
\psi(x)=\beta x^{2}+\delta x+\gamma
$$

for a Zigbee transmission. 
The differences in the form of $\psi(x)$ are due to the data rates of the respective technologies. Since the data rates for UWB and WiMax are large, they require smaller multiplicative factors in the form of return values for $\psi(x)$ to change the power cost to ensure operation within the allowable power range. Zigbee, on the other hand, with a data rate of $250 \mathrm{kbps}$ requires a much larger variation. Thus, a logarithmic function with slight increases in cost is suitable for UWB and WiMax, while a polynomial function with a larger variation in output is required for Zigbee.

While it may not seem that the benefit function in Figure 3.3(a) exhibits significant diminishing returns, when combined with the power cost function, the result is significant in affecting the optimal link utility $L_{v_{x}, v_{y}, f}^{*}$. If we refer back to Figure 3.2 , which is formed from the benefit and cost functions from Figure 3.3, we observe that the optimal link utility occurs at approximately $R_{v_{x}, v_{y}, f}^{*}=300 \mathrm{Mbps}$, only roughly $60 \%$ of the link capacity of $500 \mathrm{Mbps}$. This result points to the sensitivity of the algorithm and the need to pay careful attention to the selection of the optimization parameters in the system design process. These parameters weigh heavily in the selection of $R_{v_{x}, v_{y}, f}^{*}$ and several test runs should be performed in order to tweak the optimization parameters as necessary prior to implementation.

The optimization parameters $\alpha, \beta, \gamma$, and $\delta$ are scaling parameters for the empirical benefit and power cost. The parameter $\alpha$ scales the empirical benefit to provide a greater weight to the utility gained by achieving a higher transmission rate. On the other hand, the three scaling factors, $\beta, \gamma$ and $\delta$ are parameters in $\psi(x)$ required to scale the cost function to operate within the limits of the optimality range. Due 
to the sensitivity of the algorithm, $\alpha, \beta, \gamma$, and $\delta$ must be carefully selected. These parameters may also be used to shift the link utilities to varying degrees, depending on if $v_{x}$ and $v_{y}$ are in a sensor cluster or in the mesh network. By shifting the link utility function such that the link utilities achieved in the mesh network are higher than those in the sensor cluster, we encourage our algorithm to favor paths with more number of hops in the mesh network. This enables us to reduce the number of sensors used for routing and conserve limited sensor energies.

The link cost is updated in real-time based on the current residual energies of the transmitter $v_{x}$ and receiver $v_{y}$ of the hop, and the updated value for $\psi(E)$. As will be discussed in Chapter 5, sensors will provide feedback of their residual energies through the transmission of periodic hello messages to inform the cluster-head of its sensor state. With the arrival of the hello message at the cluster-head, the cluster-head will update the sensor's residual energy with the most recent measurement. During this time, to ensure that a prolonged duration since the last received measurement does not have an adverse effect on the optimization process, the cluster-head will extrapolate the residual energies of both $v_{x}$ and $v_{y}$; this occurs based on the elapsed time since the last received measurement and the current rate of energy dissipation of the node. In this manner, the cluster-head will have projected residual energies of $v_{x}$ and $v_{y}$ with negligible effect on the result of the optimization algorithm. Furthermore, the link costs will be updated in real-time as if the measurements were taken whenever the cost of a link changes. 


\subsection{Objective Function}

The profit function, $U_{v_{\imath}, v_{j}}^{a}(t)$, calculates the suitability of candidate path path $_{v_{\imath}, v_{\mathfrak{g}}}=$ $\left\{v_{\imath}, \ldots, v_{m}, \ldots, v_{\jmath}\right\}$ to route a message from a source sensor $v_{\imath}$ to the master node $v_{\jmath}$ through cluster-head $v_{m}$. The profit function shown in (3.6) is our objective function where the goal is to find the candidate path with the maximum profit, $U_{v_{2}, v_{\jmath}}^{a^{*}}(t)$. The profit gained by using a candidate path is calculated as the average of the optimized link utilities, $L^{*}$, across each hop $v_{x}-v_{y}, v_{x}, v_{y} \in$ path $_{v_{i}, v_{j}}$.

$$
\begin{aligned}
& U_{v_{2}, v_{3}}^{a^{*}}(t)=\max \frac{1}{K-1}\left[\sum_{x=1}^{g} L_{v_{x}, v_{x+1}, f_{x}}^{*}\left(r_{v_{x}, v_{x+1}, f_{x}}^{*}, t\right)+\sum_{y=g+1}^{K-1} L_{v_{y}, v_{y+1}, f_{y}}^{*}\left(r_{v_{y}, v_{y+1}, f_{y}}^{*}, t\right)\right] \\
& \text { s.t. (1) } P_{v_{x}, f_{x}} \cdot P_{v_{x+1}, f_{x}}=0, \quad \forall v_{x}, v_{x+1} \in \text { path }_{v_{2}, v_{y}} \\
& \text { (2) } P_{v_{y}, f_{y}} \cdot P_{v_{y+1}, f_{y}}=0, \quad \forall v_{y}, v_{y+1} \in \text { path }_{v_{2}, v_{j}} \\
& \text { (3) } \quad P_{v_{x}, f_{x}}, P_{v_{y}, f_{y}} \geq \max \left(P_{M I R S}, P_{S N R}, P_{m i n}\right), \quad \forall v_{x}, v_{y} \in \text { path }_{v_{z}, v_{3}} \\
& \text { (4) } \quad P_{v_{x}, f_{x}}, P_{v_{y}, f_{y}} \leq \min \left(P_{\text {tech }}, P_{\text {cap }}, P_{\text {max }}\right), \quad \forall v_{x}, v_{y} \in p a t v_{v_{\imath}, v_{j}} \\
& \text { (5) } \quad K^{\prime} \leq 4 \text {. }
\end{aligned}
$$

$U_{v_{i}, v_{j}}^{a^{*}}(t)$ is formed of two summations that separate the optimization of the sensor cluster and mesh network portions of a path. The first summation represents the sum of link utilities as computed via the optimized link utility function $L^{*}$ across all nodes along a candidate path inside the cluster only; $g$ represents the number of hops along the path within the cluster before reaching cluster-head $v_{m}$ and is computed as $g=f i n d\left(\right.$ path $\left._{v_{\imath}, v_{j}}==v_{m}\right)-1$. The second summation represents the sum of link utilities for a candidate path across the mesh network. Recall that there are two parts to each path, one within the sensor cluster and the other within the mesh network. 
We divide the utility sum by the hop count of the path to calculate the average link utility in the path. By doing so, we are able to more closely analyze the difference between a $k$-hop path and $(k+)$-hop path in choosing the optimal route. If we were to use total, rather than average, link utility, the algorithm would favor the $(k+)$-hop path, as the summation of more link utilities would likely lead to a higher $U_{v_{\imath}, v_{j}}^{a}$. In a power-constrained network, this over-utilizes already limited resources. Thus, by evaluating the average link utility, we are reducing latency and conserving power. Also, by finding the average link utility and limiting $K$ to a few hops, extreme link utilities (both high and low) are far more reflected in the average, and links with low utilities can be more easily identified and avoided.

If a path with more hops has the greatest average link utility $U_{v_{2}, v_{j}}^{a^{*}}$, this path will be chosen since it has the greatest trade-off of throughput performance to energy consumption across the entire path. As mentioned in Chapter 2, it is possible that a hand-off by a source sensor to a nearby node that uses a slightly longer path may traverse nodes with higher residual energies and be better for the network.

\subsubsection{Optimized Link Utility}

Our optimized link utility, $L_{v_{x}, v_{y}, f}^{*}\left(r_{v_{x}, v_{y}, f}^{*}, t\right)$ for any hop $v_{x}-v_{y}, v_{x}, v_{y} \in$ path $h_{v_{\imath}, v_{j}}$, is a function of our optimized transmission rate $r_{v_{x}, v_{y}, f}^{*}$ that is calculated from,

$$
r_{v_{x}, v_{y}, f}^{*}=\operatorname{argmax}_{R_{v_{x}, v_{y}, f}^{*}}\left[\alpha \log _{10}\left(1+\frac{R_{v_{x}, v_{y}, f}^{*}}{C_{v_{y}}(t)}\right)-\psi(E) \frac{q_{t_{x}} l R_{v_{x}, v_{y}, f}^{*}}{m}+\eta\right]
$$


where $r_{v_{x}, v_{y}, f}^{*}$ is dependent on the energy efficiency of the communication technology, $q_{t_{v_{x}}}$. Thus, the power cost includes the power drain as both a transmitter and receiver for transmitting one packet. The optimized link utility itself can be calculated as,

$$
L_{v_{x}, v_{y}, f}^{*}\left(r_{v_{x}, v_{y}, f}^{*}, t\right)=\alpha \log _{10}\left(1+\frac{r_{v_{x}, v_{y}, f}^{*}}{C_{v_{y}, f}(t)}\right)-\psi(E) \frac{q_{t_{x}} l r_{v_{x}, v_{y}, f}^{*}}{m}+\eta
$$

In the calculation of our optimized link utility $L_{v_{x}, v_{y}, f}^{*},(3.8)$ considers the empirical benefit of the receiver that would be gained by receiving throughput $r_{v_{x}, v_{y}, f}^{*}$ on channel $f$ as a fraction of the receiver's incoming capacity $C_{v_{y}, f}(t)$. The work in [92] also considers the already incoming throughput to the receiver $\sum_{\forall j^{\prime}} f_{i}^{j^{\prime}}(t)$ in their optimization in (2.4). However, since we are computing $L^{*}$ on a channel by channel basis and a node cannot receive multiple transmissions on the same channel, the incoming throughput on any available channel to a node is zero. In addition, since the cluster-head performs the resource allocation in a centralized fashion for all of its subordinate sensors, the cluster-head is able to ensure that per-channel capacity is not exceeded based on the assigned transmit powers of active transmissions. This means that flow control mechanisms to manage total network capacity are not explicitly required because cluster-heads ensure that capacity is not exceeded on a per-channel basis. As a result, total network capacity is also not violated.

Note that not all optimized hops and their corresponding $L_{v_{x}, v_{y}, f}^{*}$ values are utilized in the final path selection. All hops along each candidate simple path are optimized over all available channels and all prospective links. The optimal path is the path with 
the greatest profit value, which represents the greatest trade-off between throughput performance and energy consumption. This path will be selected for the transmission from sensor node $v_{i}$ to the master node $v_{j}$ based on the current state of the network. In the event that, after evaluating a number of candidate paths, a path cannot be resolved, the transmission request is rejected.

\subsubsection{Constraints}

The constraints on the optimization as presented in (3.6) provide the boundaries for the selection of optimal resource parameters for both sensor and mesh nodes. The first constraint, $P_{v_{x}, f_{x}} \cdot P_{v_{x+1}, f_{x}}=0, \forall v_{x}, v_{x+1} \in$ path $_{v_{\imath}, v_{3}}$, provides a power allocation restriction that a cluster node $v_{x+1}$ in an end-to-end path cannot receive and transmit on the same channel $f_{x}$, that is $f_{x} \neq f_{x+1}$. This restricts node $v_{x}$ and $v_{x+1}$, which are successive nodes in a path, from allocating power on the same transmission channel. The second constraint, $P_{v_{y}, f_{y}} \cdot P_{v_{y+1}, f_{y}}=0, \forall v_{y}, v_{y+1} \in$ path $h_{v_{2}, v_{j}}$, is the corresponding power allocation restriction for the mesh network.

The third constraint, $P_{v_{x}, f_{x}}, P_{v_{y}, f_{y}} \geq \max \left(P_{M I R S}, P_{S N R}, P_{m i n}\right), \forall v_{x}, v_{y} \in$ path $_{v_{\imath}, v_{y}}$, restricts the minimum power that the receiver can receive. $P_{M I R S}$ corresponds to the threshold input signal power at the receiver known as the minimum input receiver sensitivity (MIRS). The $P_{S N R}$ constraint is the required minimum power to reach the signal-to-noise ratio (SNR) threshold at the receiver. The third term, $P_{\min }$, represents

the lower bound on the transmit power that keeps $L_{v_{x}, v_{y}, f}^{*}\left(r_{v_{x}, v_{y}, f}^{*}, t\right)$ positive. There 
is a fourth factor that we ignore and that is the lower bound on the available capacity at the transmitter, which is zero and is thus ignored.

The fourth constraint, $P_{v_{x}, f_{x}}, P_{v_{y}, f_{y}} \leq \min \left(P_{t e c h}, P_{c a p}, P_{\max }\right), \forall v_{x}, v_{y} \in$ path $_{v_{2}, v_{y}}$ restricts the maximum power of the optimality range. $P_{\text {tech }}$ corresponds to the maximum allowable transmit power on a transmission channel for the technology being used. $P_{c a p}$ represents the power that corresponds to the available outgoing capacity on the channel at the transmitter and ensures that the channel capacity is not exceeded. While the lower bound on the outgoing capacity is zero in the previous constraint, the upper bound $P_{c a p}$ is not zero unless the full channel capacity is being used by the transmitter. Meanwhile, $P_{\max }$ restricts the upper bound on transmit power as that which keeps $L_{v_{x}, v_{y}, f}^{*}\left(r_{v_{x}, v_{y}, f}^{*}, t\right)$ positive.

It is interesting to note that, in our study, we do not model an explicit transmitter utility function. In [92], Wang and Li use the roots $\left(r_{\min }^{j}, r_{\max }^{j}\right)$ of the transmitter utility function to find the range of throughput that keeps the transmitter $j$ 's utility function positive. These roots are computed given the transmitter's service price that it is charging at the time; at high prices, the transmitter will make its entire capacity available as it gains more revenue by charging a higher price per unit of throughput, while at low prices, the transmitter may only make a small portion of its capacity available, if any. However, in our study, transmitters do not gain revenue by making themselves available in a multi-hop path as we only look at the power costs of using a link from the perspective of a candidate receiver. Thus, in our study, the transmitter is forced to make its full capacity available to a receiver, and $P_{\text {cap }}$ corresponds to the 
transmit power necessary to achieve the upper bound of this available capacity. The available capacity is denoted as $C_{v_{x}, f}^{a v a l l}$, where $C_{v_{x}, f}^{a v a a l}=C_{v_{x}, f}-\sum_{v_{k} \in U_{v_{x}, f}(t)} R_{v_{x}, v_{k}, f}$ is the available capacity of node $v_{x}$ on channel $f, C_{v_{x}, f}$ is the total capacity of $v_{x}$ on that channel, $U_{v_{x}, f}(t)$ is the transmitter's set of outgoing nodes using channel $f$ in time slot $t$, and $R_{v_{x}, v_{k}, f}$ is the current transmission rate from $v_{x}$ to an outgoing node $v_{k}$ of an active transmission.

The final constraint limits the number of hops in a path to $K^{\prime} \leq 4$. As mentioned, this limits the number of nodes whose power is dissipated in a single path.

\subsection{Radio Models for Wireless Technologies}

Our sensor and mesh nodes operate in the $2.4-11 \mathrm{GHz}$ band based on the band of operation for the Zigbee cluster, UWB cluster and WiMax mesh network. Table 3.1 summaries the band characteristics and other operating parameters for these three networks. For our antenna gains, each of our Zigbee and UWB nodes utilize a single $\mathrm{Tx} / \mathrm{Rx}$ antenna with gains of $0 \mathrm{dBi}$ and $3 \mathrm{dBi}$, respectively. Our WiMax mesh nodes have transmit and receive antenna gains of $13 \mathrm{dBi}$ and $16 \mathrm{dBi}$, respectively.

Table 3.1: Comparison of Operating Parameters for Zigbee, UWB and WiMax Nodes [22][100][101][102]

\begin{tabular}{cccccc}
\hline \hline Standard & Band of Operation & $\begin{array}{c}\text { Channel } \\
\text { Bandwidth }\end{array}$ & MIRS & $\begin{array}{c}\text { Minimum } \\
\text { SNR }\end{array}$ & Maximum EIRP \\
\hline Zigbee & $2.4-2.5 \mathrm{GHz}$ & $5 \mathrm{MHz}$ & $-85 \mathrm{dBm}$ & $-1.2 \mathrm{~dB}$ & $0 \mathrm{dBm}$ \\
UWB & $3.0-11 \mathrm{GHz}$ & $500 \mathrm{MHz}$ & $-85 \mathrm{dBm}$ & $-1.59 \mathrm{~dB}$ & $-14.3 \mathrm{dBm}$ \\
WiMax & $5.25-5.725 \mathrm{GHz}$ & $10 \mathrm{MHz}$ & $-83.2 \mathrm{dBm}$ & $9.8 \mathrm{~dB}$ & $24 \mathrm{dBm}$ \\
\hline
\end{tabular}


The maximum Effective Isotropic Radiated Power (EIRP) presented in the table is the maximum power that can be put on the transmission channel, and thus includes the transmit antenna gain. As a result, in terms of our constraints, $P_{\text {tech }}(\mathrm{dBm})$ $=$ Maximum EIRP $(\mathrm{dBm})-G_{t}(\mathrm{dBi})$ is the maximum transmit power that can be allocated to a channel prior to applying the antenna gain. The constraints $P_{S N R}$ and $P_{M I R S}$ are computed directly from the operating parameters provided in the table.

\subsubsection{Path Loss}

The next consideration is the path loss where we consider indoor environments for our Zigbee and UWB clusters with LOS conditions, while we consider long-haul and NLOS conditions for our WiMax mesh nodes that transmit over longer distances. We use the Friis equation to compute the path loss,

$$
P L=32.45+20 \log _{10}(f)(M H z)+10 n \log _{10}(d)(k m)
$$

where $n$ specifies the path loss exponent for the environment. We utilize $n=1.8$ for our UWB cluster due to the proximity of nodes to one another and $n=2.0$ for our Zigbee cluster. We use $n=2.2$ for our long-haul WiMax mesh network. If the distributed WSN is used for outdoor sensory applications, this will affect the path loss model and may require a denser network with shorter distances between nodes to compensate. During the setup of each network, the network will also be separately calibrated to determine the right values for the optimization parameters $\alpha, \beta, \gamma, \delta$ 
and $\eta$ for our objective function based on factors such as the path loss models selected and per-hop throughput, among others This ensures successful operation and will be covered further in Chapter 5

A sample link budget analysis for all three technologies is presented in Table B 1 in Appendix B The Rx Sensitivity (also denoted as MIRS) is computed in (3 10) using the UWB parameters from Table B 1 as an example

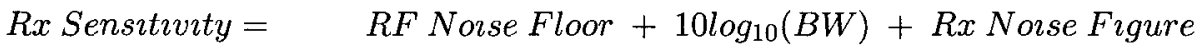

$$
\begin{aligned}
& + \text { Required } E_{f} / N_{0}+\text { Implementation Loss } \\
& =\quad-174 \mathrm{dBm} / \mathrm{Hz}+10 \log _{10}(500 \mathrm{MHz})+060 \\
& -159+30 \\
& =\quad-85 \mathrm{dBm}
\end{aligned}
$$

\subsection{Summary}

This chapter discussed the formulation of our throughput and problem for applicationcentrıc WSNs In doing so, two contributions of this thesis were presented Firstly, a microeconomic solution to define the link suitability was derıved in terms the achievable throughput and associated power cost This was performed in terms of the consumption of PHY layer resources and transceiver power consumption The link suitabihty presents a clear picture of the impact of PHY layer resources on throughput and energy capacity By using the link suitability, we are able to identıfy transmission channels with the highest trade-off between throughput and energy consumption such that we can allocate resources efficiently in application-centric WSNs 
Secondly, our cross-layer optimization policy was presented that jointly considers functions at the PHY, link and routing layers. The goal is to identify the optimal routing path with the highest trade-off between throughput benefits and energy costs across multiple transmission channcls. Wc are the first, to the best of our knowledge, to design a cross-layer optimization protocol for application-centric WSNs with the goal of maximizing the trade-off between throughput performance and energy consumption as a function of common physical resources for resource allocation. Wo have submitted our findings, and the formulation of our link suitability and cross-layer optimization policy, for publication in a technical article in [103].

This chapter also provided our formal problem definition, the underlying assumptions for our study, and the radio models that form the basis of our wircless communication. The next chapter will prescnt our cross-layer design and explain the functions at each layer. This will include a discussion of the channel scheduling algorithm that is a function specific to the link layer. 


\section{Chapter 4}

\section{Cross-Layer Design}

\subsection{Introduction}

The cross-layer optimization policy for application-centric WSNs is designed to jointly satisfy the requirements of application performance and energy efficiency in nextgeneration networks. Our cross-layer design focuses on functions at three layers: the PHY, link and network layers. At the PHY layer, we aim to maximize the usage of PHY layer resources in considering both the benefits and costs of resource consumption in terms of throughput and energy dissipation, respectively. To do so, our cross-layer optimization policy maximizes the link utility function of the receiver in a negotiation for the optimal transmit power and transmission rate for a hop along a candidate path. At the link layer, we aim to optimize the channel selection by looking at interference levels and preferred operating channels of prospective receivers. As a result, we aim to find the optimal channel assignment for nodes along the path to efficiently use available capacity. Finally, at the network layer, our goal is to 
determine the candidate path with the greatest suitability, or profit, over all hops considering our optimization constraints. In addition, the network layer aims to favor routing through the mesh network. By performing this cross-layer optimization, we can design next-generation WSNs that are application-centric yet power aware.

This chapter presents our cross-layer design and discusses the functions of the PHY, link and network layers and their contributions to solving the throughput problem. From this point onwards, we will use $M=\left|M_{\imath}\right|$ and $V=\left|V_{\imath}\right|$ to represent the cardinalities of the mesh and sensor node sets, respectively, to simplify equations.

\subsection{Protocol Stack Design}

The design of a cross-layer optimization algorithm requires efficient communication between protocol stack layers such as the PHY, link, network and application layers. Our protocol stack design follows the direct signaling method between non-adjacent layers presented in [32]. The direct signaling method reduces latency in the communication between multiple layers and is crucial in the design of cross-layer optimization algorithms. The direct signaling scheme for our protocol stack model is illustrated in Figure 4.1 and presents the cross-layer considerations used in this thesis.

The goal of direct signaling is to exchange information between important protocol layers such that required information to perform cross-layer optimization is retrieved, and allocation decisions are sent, with minimal delay. For example, the clusterhead's PHY layer will inform the application layer of the sensor and mesh node 


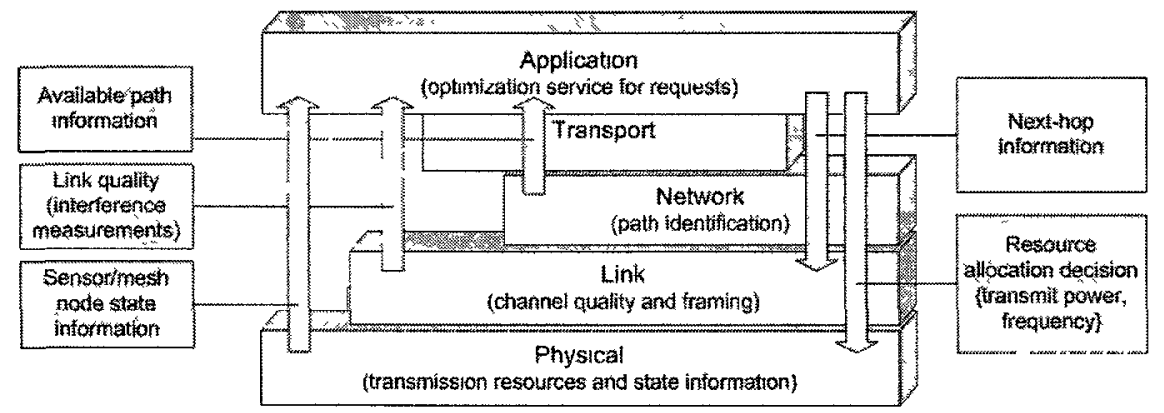

Figure 4.1: Protocol Stack Model for Application-Centric WSNs

state information which includes energy rating information, surrounding interference and more. State information and coordination protocols to provide feedback to the cluster-head are covered in Chapter 5. The link layer and network layer will also inform the application layer of the channel conditions and available path information, respectively. Furthermore, upon executing the cross-layer optimization policy, the application layer will inform the PHY layer of the necessary resource allocations and the link layer of the next-hop information. These interactions require minimal delay in traversing the protocol stack for optimized and timely responses.

For any two non-adjacent layers, $l_{x}$ and $l_{y}$, the propagation latency $T_{l_{x}, l_{y}}^{D S}$ for the direct signaling method is calculated as in [32],

$$
T_{l_{x}, l_{y}}^{D S}=\frac{T_{l_{x}, l_{y}}^{L}}{(n-1)}
$$

where $T_{l_{x}, l_{y}}^{L}$ is the propagation latency between layers $l_{x}$ and $l_{y}$ in a traditional layered protocol stack with $(n-1)$ layers between them. Hence, the direct signaling method provides a speed-up factor of $(n-1)$ [32]. 


\subsection{Node States}

At any time, a cluster-head is in one of six states:

- Initialization State: At the onset of our simulation, the initialization phase of the cluster-head computes the paths from all sensors to the master node using our Simple Path Protocol (SPP) that will be covered in Section 4.4.1. This algorithm gathers all paths in the network from individual sensors through the mesh network to the master node that contain at most $K^{\prime}$ hops. These paths are stored in the $\left(K^{\prime}+1\right) \times W \times V$ path matrix, $X$;

- Link Optimization State: While servicing a request, the cluster-head optimizes all link utilities $L^{*}$ that exist along candidate paths for a single source sensor. To determine this set of links, the cluster-head forms the $\left(K^{\prime}+1\right) \times W$ sub-matrix, $A \subset X$, to gather available paths for the given source sensor;

- Channel Optimization State: While servicing a request, the cluster-head finds the most preferred channels, $f_{1}^{*}, \ldots, f_{K^{\prime}}^{*}$, along a candidate path $a \in A$ from source sensor $v_{\imath}$ to $v_{\jmath}$ by ranking channel bins according to their link utilities. The cluster-head attempts to allocate the most preferred bins to each hop along the path. As it may not be possible to select the most preferred bin allocation due to channel constraints, it may be necessary to iterate over possible bin combinations to find the valid combination with the largest suitability coefficient over all links. In this manner, the cluster-head assigns an overall 
suitability coefficient or profit, $U_{v_{\imath}, v_{\jmath}}^{a}$, to each candidate path. $U_{v_{\imath}, v_{j}}^{a}$ is formed as the average link utilities using the optimal channel allocation for the path;

- Path Optimization State: In allocating a path to service a request, the cluster-head ranks the candidate paths in order of their suitabilities, $U_{v_{2}, v_{\jmath}}^{a}$, selects the candidate path with the highest suitability and denotes it as $U_{v_{2}, v_{j}}^{a^{*}}$;

- Transmission State: If the cluster-head is relaying packets for its subordinate sensors to the mesh network en route to the master node, the cluster-head is in the transmission state; and,

- Sleep State: If the cluster-head is not servicing a request or relaying traffic, the cluster-head is asleep and conserving energy in a low power state.

Individual sensor nodes are either in sensing state, transmitting state to send traffic to the next hop either as the source or relay node, or sleep state. Mesh nodes that are not assigned to clusters can be in transmission state or sleep state. The roles of each layer and node functions in these states will be discussed next.

\subsection{Network Layer}

The network layer focuses on routing functions at the cluster-head, which is responsible for computing the path for communication from the source sensor to the master node at the time of a request. Network layer functions take place in two states: the Initialization State and the Path Optimization State. In the Initialization State, the 
available paths that exist in the network are found. The paths in a distributed WSN are determined and stored at the cluster-head using a centralized path algorithm. The paths are not maintained at the sensors in a decentralized fashion as sensors are generally memory-constrained. In the Path Optimization State, a number of candidate paths with overall suitabilities $\left\{U_{v_{2}, v_{j}}^{a}\right\}$ are compared and an optimal path with suitability $U_{v_{\imath}, v_{j}}^{a^{*}}$ is chosen.

\subsubsection{Initialization State}

In the initialization phase, the list of paths from sensors in both of our clusters to the master node is determined. Rather than computing the available paths from each source to the master node individually, we present a destination-initiated simple path algorithm known as the Simple Path Protocol (SPP) that propagates knowledge of paths through a network graph; a simple path, by definition, is a path where nodes are traversed at most once. We avoid routing loops by limiting the propagation of path knowledge to nodes that are not already in a known path.

\section{Simple Path Protocol (SPP)}

The goal of SPP is to determine the set of paths in a directed network graph that contains routing loops. During the Initialization Phase of a newly deployed network, all sensor nodes will have equal initial energy capacities. However, it is likely that, at any stage during a network's lifetime, node sets will have an unequal distribution of energy capacities. This can occur through normal operation based on variations 


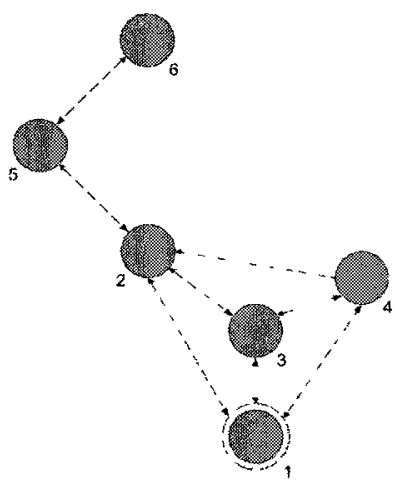

(a) A mesh network with loops and directed links

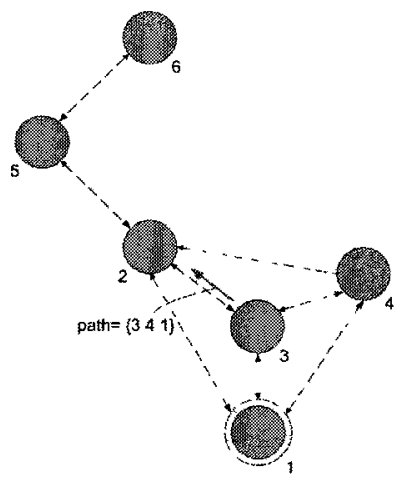

(d) Resultant new path advertisement from $\left\{\begin{array}{ll}4 & 1\end{array}\right\}$ at Node 3 only

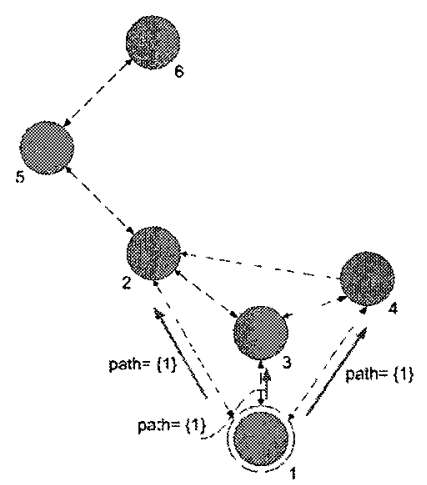

(b) Initial master node path advertisement

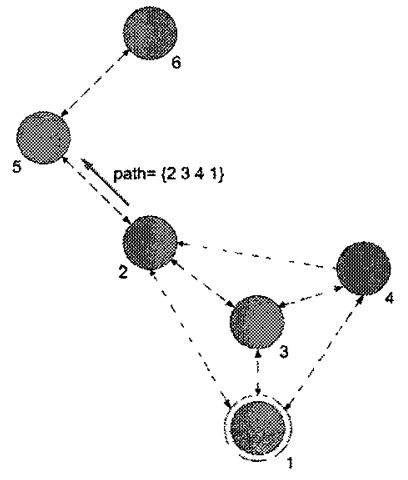

(e) Path advertisement of $\{3$ 41 \} handled at Node 2

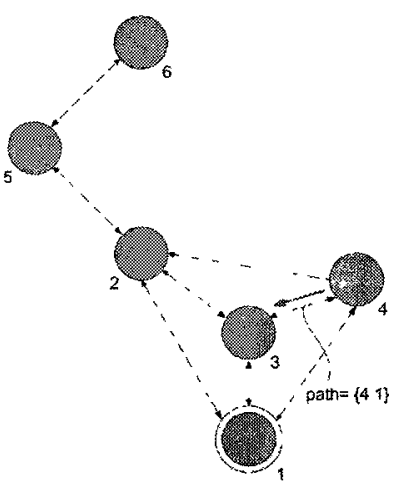

(c) New path advertisement for Node 4 only

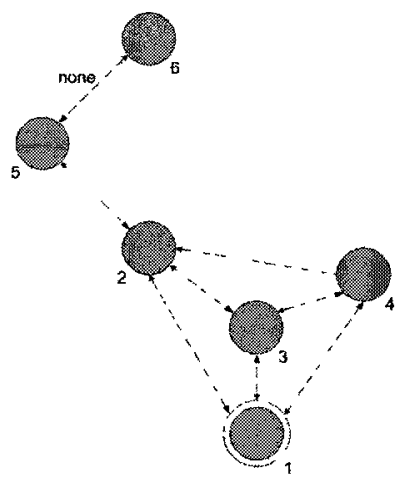

(f) Path advertisement handled at Node 5 for path $_{5,1}=$ $\left\{\begin{array}{lllll}5 & 2 & 3 & 4 & 1\end{array}\right\}$

Figure 4.2: New Path Advertisements in Simple Path Protocol (SPP)

in traffic carried in the network, the energy capacities of a number of nodes being replenished or new nodes joining the network at any time. Thus, these varying resource availabilities cannot be ignored in sensor or mesh networks and a directed graph must be considered. In our work, the centralized controller that executes SPP is the cluster-head that is responsible for optimizing sensor requests. However, prior to optimization, all available paths with a maximum of $K^{\prime}$ hops are determined. 
Consider an example of a mesh network with $M=5$ nodes shown in Figure 4.2(a) that consists of directional and bi-directional links. The corresponding binary connectivity matrix, $C\left(v_{x}, v_{y}\right)$, is given in (4.2). $C\left(v_{x}, v_{y}\right)$ has a Bernoulli distribution where $C\left(v_{x}, v_{y}\right)=1$ with success probability $p$ if $v_{y}$ is in range of $v_{x}$, and $C\left(v_{x}, v_{y}\right)=0$ with failure probability $(1-p)$, otherwise. It is required by the SPP algorithm that all links in valid paths are bi-directional such that traffic can successfully flow in both directions; while data must certainly flow from the source sensor to the master node, the required resource allocation computed by the cluster-head during crosslayer optimization must also be able to reach the source sensor in the opposite path direction prior to transmission.

$$
C\left(v_{x}, v_{y}\right)=\left[\begin{array}{llllll}
0 & 1 & 1 & 1 & 0 & 0 \\
1 & 0 & 1 & 0 & 1 & 0 \\
1 & 1 & 0 & 1 & 0 & 0 \\
1 & 1 & 1 & 0 & 0 & 0 \\
0 & 1 & 0 & 0 & 0 & 1 \\
0 & 0 & 0 & 0 & 1 & 0
\end{array}\right]
$$

Sincc the SPP algorithm is a destination-initiated protocol, the initial stcp of the algorithm requires the destination node, in our case the master node, to begin the algorithm by announcing the start of the algorithm to its immediate neighbors. Given our requirement for bi-directional links, the valid set of neighbors for SPP are those for whom only bi-directional links exist. Thus, in Figure 4.2(a), all links except $\{2$ $4\}$ and $\left\{\begin{array}{ll}42 \\ 4\end{array}\right.$ are valid as only node 4 can transmit to node 2 . We can retrieve the 
valid set of neighbors for any given node $v_{x}$ by determining at which positions both $C\left(v_{x},:\right)=1$ and $C\left(:, v_{x}\right)=1$.

If we extend our example to consider path advertisements in Figure 4.2(b), consider that the master node (node 1) creates its initial path advertisement consisting of its own node ID, in this case path $_{2 n t}=\{1\}$. This advertisement is read by the master node's immediate neighbors (nodes 2, 3 and 4). These nodes will place their own node IDs at the front of the master node's path advertisement, forming path $_{2,1}$ $=\left\{\begin{array}{ll}2 & 1\end{array}\right\}$ and $\operatorname{path}_{3,1}=\left\{\begin{array}{ll}3 & 1\end{array}\right\}$ and path $_{4,1}=\left\{\begin{array}{ll}4 & 1\end{array}\right\}$, respectively. An important aspect of the SPP algorithm is that new path advertisements of a node are not advertised to the neighbor from which the path advertisement was received, the master node or any neighbor of the node that already exists within the path advertisement. This reduces unnecessary path advertisements (i.e. one that would yield a loop or one that would require the hop count to exceed $K^{\prime}$ hops).

For example, let us consider the propagation of a single path advertisement where node 4 sends path advertisement path $h_{4,1}=\left\{\begin{array}{ll}4 & 1\end{array}\right\}$ to its immediate neighbors. Node 4's valid immediate neighbors (nodes 1 and 3 ) are formed where both $C(4,:)=1$ and $C(:, 4)=1$; as mentioned, because node 4 does not have a bi-directional link with node 2 , node 2 is not a valid immediate neighbor. At the next iteration, only node 3 will receive advertisement path $_{4,1}=\left\{\begin{array}{l}4 \\ 1\end{array}\right\}$ as shown in Figure 4.2(c) because node 1 is the master node. If we follow what happens with the path advertisement at node 3 only as in Figure 4.2(d), at the next iteration node 3 will send path advertisement path $_{3,1}=\{341\}$ to node 2. Although node 3's immediate neighbors are nodes 1,2 , 
and 4 , since node 1 is the master node and node 4 is already in the path, only node 2 will receive this new path advertisement; sending advertisement path $_{3,1}=\left\{\begin{array}{lll}3 & 4 & 1\end{array}\right\}$ to node 4 would yield a new path advertisement of $p^{2} h_{4,1}=\left\{\begin{array}{llll}4 & 3 & 4 & 1\end{array}\right\}$ that would create an undesired routing loop wasting already constrained sensor resources.

When node 2 receives path advertisement path $_{3,1}=\left\{\begin{array}{ll}34 & 1\end{array}\right\}$, it appends its node ID at the front of this path advertisement at the next iteration to form path $_{2,1}=\{2$ $341\}$ as in Figure 4.2(e). When node 2 determines its immediate neighbors as nodes 1,3 and 5 , only node 5 will receive the advertisement, yielding a path path $_{5,1}=\{5$ 2341 with the maximum number of hops $K^{\prime}=4$. Since adding any node to this path would exceed $K^{\prime}$, this path is not advertised by node 5, shown in Figure 4.2(f). SPP propagates new path advertisements until all new paths have been advertised and forwarded. By following this strategy, we can efficiently determine all candidate paths from all sources to a single destination of maximum $K^{\prime}$ hops in a directed network graph.

\section{Time Complexity}

In centralized networks, such is the case in distributed WSNs where cluster-heads are the decision makers, the importance of the cluster-head determining the optimal route for a sensor is known. For this purpose, we will identify two important concepts that go hand-in-hand with route determination: route availability and route evaluation. Route availability observes the state of connectivity, while route evaluation determines the optimal path from one or more available paths. 
There are two distinct design alternatives that can be considered for route determination. Standard practice is to maintain a connectivity matrix that is updated on an ongoing basis through signaling that highlights the liveness of the nodes in the network. The connectivity matrix is maintained in either a centralized fashion at a cluster-head, for example, or each node maintains a neighbor list. Once the connectivity matrix is known, route evaluation is performed by executing an algorithm at the time of a request such as the shortest cost Dijkstra's algorithm that has a time complexity of $O\left(V^{2}\right)$ using an ordinary linked list or array [104].

For sensor network applications, link costs, in our case the link utilities $L$, are dynamic and a function of one or more parameters. For example, the cost of using a link is a function of residual energy capacities of the transmitter and receiver of the hop, the transmit power required and bandwidth used for the transmission. However, shortest cost path methods such as Dijkstra's algorithm would have to be repeated whenever there is a change to a link cost in the network. The shortest cost path would also have to be recalculated for each new source request as Dijkstra's algorithm is a single source algorithm. Hence, the time complexity considering all sources each with $X_{i}$ requests is $O\left(V^{3} X_{i}\right)$. Given our network, such an algorithm would have to iterate over a number of resource allocations for multiple candidate paths per sensor request.

The second approach is to abstract the route availability function into the initialization phase of the network, which provides the motivation for the SPP. Thus, the cluster-head simply has to access the path matrix in real-time during request handling as route availability has already been performed, which simplifies the optimization 
process. These available paths will be consistent with the real-time connectivity of the network, as the cluster-head will activate and de-activate paths on an ongoing basis based on signaling in the network. This will be covered in more detail shortly. Abstracting the route availability to the initialization phase has a time complexity of $O\left(\min \left(L_{h},\left(K^{\prime}+1\right)\right)\right)$ and is required to be run once where $L_{h}$ is the maximum hop count away from the master node.

The time complexity considers that the algorithm is divided into fixed time intervals within which nodes receive path updates from their neighbors. To start SPP, the master node broadcasts the master node ID 1. At the end of the first interval, the immediate neighbors of the master node will append their node IDs to the master node broadcast for a path length of two for interval two. At the end of each subsequent interval, each node announces its new paths by appending its own node ID to all received paths during the interval. However, the node will only append its own ID to the path and broadcast the new path if it satisfies the maximum hop count $K^{\prime}$ and the node ID is not yet in the path. Thus at each interval $k$, each node will only process path lengths of length $k$. This occurs for $\left(K^{\prime}+1\right) \leq L_{h}$. If $\left(K^{\prime}+1\right)>L_{h}$, the time complexity depends on $L_{h}$. It is important to note that it is the maximum hop count away from the master node and not the number of nodes $|Z|$ in the sensor and mesh networks directly that contributes to the time complexity. Consider a case where $|Z|=1,000$ nodes are connected to the master node only for which $L_{h}=1$; regardless of the size of $\left(K^{\prime}+1\right)$ or $|Z|$, the algorithm will complete in $O(1)$. However, the number of nodes $|Z|$ in multi-hop networks does generally affect the value of $L_{h}$. 
The purpose of abstracting route availability to the initialization phase is to simplify the route evaluation process at the time of a request. By simply evaluating a number of pre-known paths at run-time as opposed to evaluating a full network graph at each request, the time complexity of the run-time algorithm is significantly reduced. In fact, we find that the time complexity is linear with $O\left(V X_{\imath}\right)$. Thus, the overall time complexity including route availability is $O\left(\min \left(L_{h},\left(K^{\prime}+1\right)\right)+V X_{\imath}\right)$. Such an approach is crucial for next-generation WSN applications such as those for safety or military applications that require immediate responses.

\section{Merging the Connection Matrix}

The maximum number of hops $K^{\prime}$ along the path requires us to merge the connection matrices of each cluster individually with the connection matrix of the mesh network such that we can enforce $K^{\prime}$ end-to-end along the path. This allows us to find $W$ available paths that originate from sensors within a single cluster to the mesh node. Consider a snap-shot of the $M \mathrm{x} M$ connection matrix $C_{m}\left(v_{a}, v_{b}\right)$ for the mesh network that has experienced link outages for which $M=7$ in (4.3).

$$
C_{m}\left(v_{a}, v_{b}\right)=\left[\begin{array}{ccccccc}
0 & 1 & 0 & 0 & 0 & 1 & 1 \\
1 & 0 & 1 & 0 & 0 & 0 & 1 \\
0 & 1 & 0 & 1 & 0 & 0 & 1 \\
0 & 0 & 1 & 0 & 1 & 0 & 1 \\
0 & 0 & 0 & 1 & 0 & 1 & 1 \\
1 & 0 & 0 & 0 & 1 & 0 & 1 \\
1 & 1 & 1 & 1 & 1 & 1 & 0
\end{array}\right]
$$




$$
C_{c}\left(v_{c}, v_{d}\right)=\left[\begin{array}{ccccc}
0 & 0 & \ldots & \ldots & c_{1 v} \\
1 & 0 & \ldots & \ldots & c_{2 v} \\
\vdots & \vdots & \ddots & & \vdots \\
\vdots & \vdots & & \ddots & \vdots \\
c_{v 1} & c_{v 2} & \ldots & \ldots & c_{v v}
\end{array}\right]_{V x V}
$$

Also, consider an arbitrary $V \mathrm{x} V$ connection matrix, $C_{c}\left(v_{c}, v_{d}\right)$, for a single cluster consisting of $V-1$ sensors and a cluster-head in (4.4). Based on the number of nodes $V$ in the cluster, the cluster-head will always exist at index $V$ in $C_{c}$. However, the cluster-head can have any ID in the mesh. Thus, to enforce a restriction on the maximum number of hops for a single path, we must merge $C_{c}$ and $C_{m}$ to form a global view of connectivity in the network. However, because the cluster-head can exist in any position in the mesh network, we must first manipulate $C_{m}$ to ensure consistency in the merged matrix.

The approach we use is to create a temporary mesh connection matrix, $C_{m, t e m p}$, that re-arranges the mesh connection matrix such that the cluster-head of interest exists in row one. This allows us to accommodate multiple clusters and create a generic algorithm that can support different cluster sizes.

Consider that the cluster-head in $C_{c}$ exists in the $m^{\text {th }}$ row in $C_{m}$, i.e. has a mesh ID of $m$. The cluster-head exists in both matrices to represent the available connections for the cluster-head within the cluster and mesh, respectively. Thus, the cluster-head will have both a cluster ID of $V$ and a mesh ID of $m$. To form $C_{m, t e m p}$, all nodes in the 
mesh network are given temporary node IDs such that the cluster-head in the mesh is given temporary ID 1 . Mesh nodes one through $m-1$ in $C_{m}$ are given temporary IDs two through $m$, while all nodes $m+1$ to $M$ are given temporary IDs that are identical to their true IDs. Hence, for all nodes whose actual IDs are greater than the actual ID of the cluster-head $m$ in the mesh, their IDs will not change.

For example, consider a situation where our cluster-head has an actual mesh ID of $m=5$ and, thus, exists in row and column five in the original $C_{m}$ matrix. After the temporary ID assignment, it will be given temporary ID 1 and placed in the first row and column of $C_{m, t e m p}$. If we consider the master node's position in $C_{m}$, for example, if the master node's ID $a>m$, the master node's temporary ID will equal its actual ID. Otherwise, if the master node's actual mesh ID $a<m$, it will be given temporary ID $a+1$. Thus, if say $a=3$, after the temporary ID assignment, the master node will be given temporary ID 4 . With this approach, it is possible to determine the paths between sensors and the master node for different clusters using temporary identifiers.

To update the connections in the matrix, we re-observe the transmission ranges of each node and compare the distances between the nodes; if the distance between any two nodes is less than or equal to the range, the connection is given a value of 1 , otherwise, it is simply given a value of 0 . We form the $C_{m, t e m p}$ connection matrix in (4.5) considering the temporary ID assignment considered above for the actual cluster-head and master node IDs of $m=5$ and $a=3$, respectively. Recall that the cluster-head will be given temporary ID 1 and the master node will be given temporary ID 4 , and will exist in these rows in $C_{m, t e m p}$, respectively. 


$$
C_{m, t e m p}\left(v_{a}, v_{b}\right)=\left[\begin{array}{ccccccc}
0 & 0 & 0 & 0 & 1 & 1 & 1 \\
0 & 0 & 1 & 0 & 0 & 1 & 1 \\
0 & 1 & 0 & 1 & 0 & 0 & 1 \\
0 & 0 & 1 & 0 & 1 & 0 & 1 \\
1 & 0 & 0 & 1 & 0 & 0 & 1 \\
1 & 1 & 0 & 0 & 0 & 0 & 1 \\
1 & 1 & 1 & 1 & 1 & 1 & 0
\end{array}\right]
$$

Given that the index of the cluster-head is now in position one, we can merge $C_{m, t e m p}$ and $C_{c}$ to globally enforce our maximum hop constraint. We form our final merged $(M+V-1) \times(M+V-1)$ matrix $C_{\text {merged }}$ in $(4.6)$ to represent the overall binary connection matrix between a single cluster and the mesh. This allows us to find end-to-end paths from a source sensor to the master node, enforcing a $K^{\prime}$ maximum hop constraint for all paths using SPP. Whatever the master node's temporary ID $r$ is in the $C_{m, t e m p}$ connection matrix, the master node can be found at index $(r+V-1)$ in $C_{\text {merged }}$. This new mesh index is used as the initıal identifier to start SPP.

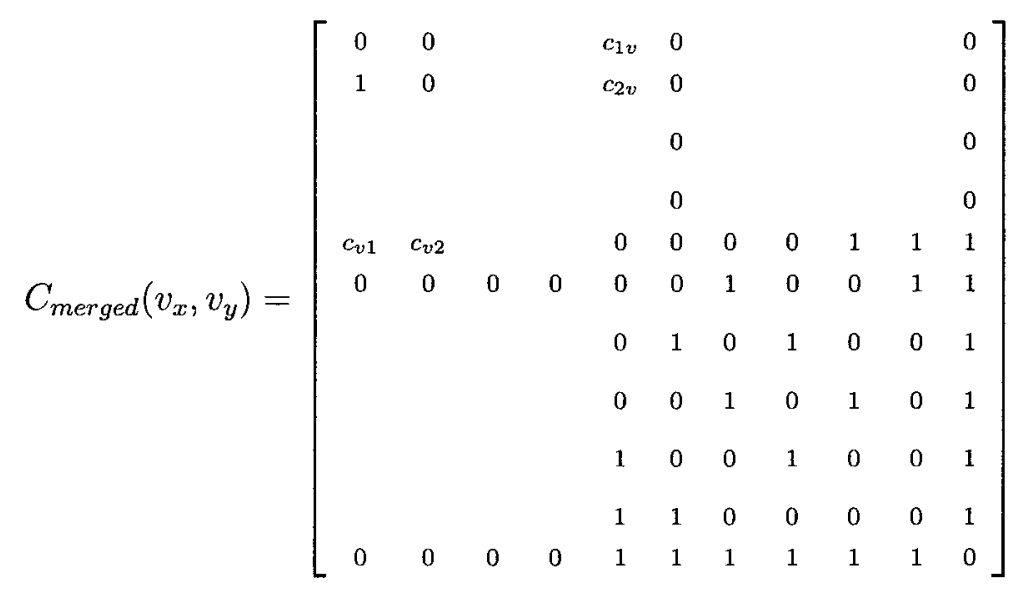


Upon forming $C_{\text {merged }}$, we use the destination-initiated SPP protocol branching out from the master node to distribute new path advertisements throughout the network graph. We execute this process for each cluster in the network because, as stated, sensor clusters will not communicate with one another. Thus, for multiple clusters in a distributed WSN, each cluster-head will execute the SPP individually.

The expected maximum number of paths over all sensors, $E[W]$, which we defined in Chapter 3, is computed as,

$$
\begin{aligned}
E[W] & =p+\sum_{K=2}^{K^{\prime}}\left(\begin{array}{c}
M+V-3 \\
K-1
\end{array}\right) p^{K} \\
& =p+\sum_{K=2}^{K^{\prime}}\left(\frac{(M+V-3) !}{(K-1) !(M+V-K-2) !}\right) p^{K}
\end{aligned}
$$

where $p^{K}$ is the probability that a single $K$-hop path exists and $\left(\begin{array}{c}M+V-3 \\ K-1\end{array}\right)$ is our binomial coefficient. It enforces our loop-free condition of a path that $(K-1)$ unique nodes must be selected from $(M+V-3)$ possible next hops, excluding the source and destination, to form a valid candidate path. Note that, with a successful probability of connection $p$, there are only $p$ paths with one hop.

\subsubsection{Path Optimization State}

In the path optimization state, all candidate paths determined from (4.6) using the SPP protocol are given an overall suitability coefficient, $U_{v_{\imath}, v_{j}}^{a} \cdot U_{v_{\imath}, v_{\jmath}}^{a}$ for candidate path $a$ is computed in (4.8) as the average of the optimal link utility values computed 


$$
\begin{aligned}
& U_{v_{2}, v_{y}}^{a^{*}}(t)=\max \frac{1}{K-1}\left[\sum_{x=1}^{g} L_{v_{x}, v_{x+1}, f_{x}}^{*}\left(r_{v_{x}, v_{x+1}, f_{x}}^{*}, t\right)+\sum_{y=g+1}^{K-1} L_{v_{y}, v_{y+1}, f_{y}}^{*}\left(r_{v_{y}, v_{y+1}, f_{y}}^{*}, t\right)\right] \\
& \text { s.t. (1) } P_{v_{x}, f_{x}} \cdot P_{v_{x+1}, f_{x}}=0 \\
& \text { (2) } P_{v_{y}, f_{y}} \cdot P_{v_{y+1}, f_{y}}=0 \\
& \forall v_{x}, v_{x+1} \in \text { path }_{v_{\imath}, v_{3}} \\
& \forall v_{y}, v_{y+1} \in \text { path }_{v_{\imath}, v_{3}} \\
& \text { (3) } \quad P_{v_{x}, f_{x}}, P_{v_{y}, f_{y}} \geq \max \left(P_{M I R S}, P_{S N R}, P_{m \imath n}\right), \quad \forall v_{x}, v_{y} \in \operatorname{path}_{v_{i}, v_{j}} \\
& \text { (4) } \quad P_{v_{x}, f_{x}}, P_{v_{y}, f_{y}} \leq \min \left(P_{t e c h}, P_{c a p}, P_{m a x}\right), \quad \forall v_{x}, v_{y} \in \text { path }_{v_{2}, v_{j}} \\
& \text { (5) } \quad K^{\prime} \leq 4 \text {. }
\end{aligned}
$$

via $L_{v_{x}, v_{y}, f}^{*}$ using the optimal channels selected at the link layer for hop $v_{x}, v_{y} \in a$. In this section, we will use the terms $L_{v_{x}, v_{y}, f}^{*}$ and $L^{*}$ interchangeably.

Upon evaluating the suitability coefficients for all paths, $\left\{U_{v_{\imath}, v_{g}}^{a}\right\}_{1 \times W}$, where $W$ was defined as the maximum number of available paths over all nodes in the path matrix $X$, the optimal path has the largest suitability coefficient, $U_{v_{2}, v_{3}}^{a^{*}}$. This path from source sensor $v_{\imath}$ to master node $v_{\jmath}$ satisfies the constraints presented in the objective function and is selected for the transmission.

\section{Handling Lost Links and Nodes}

For the improved survivability of our system, our network must be able to recover from lost links and nodes due to malfunction, attacks or intrusions. Thus, upon receiving a signal for a lost link or node connection in the network, the cluster-head will perform two tasks:

- Update Connection Matrix: For a lost link $v_{x}-v_{y}$, characterized by a timeout of one second for an active transmission, the cluster-head will access either the sensor or mesh connection matrix depending on the sub-network 
in which $v_{x}-v_{y}$ resided. The cluster-head will subsequently update either $C_{c}\left(v_{x}, v_{y}\right)=0$ or $C_{m}\left(v_{x}, v_{y}\right)=0$ to reflect the lost connection. For a lost node sensor $v_{x}$, the cluster-head will update $C_{c}\left(v_{x},:\right)=0$ and $C_{c}\left(:, v_{x}\right)=0$; likewise, if $v_{x}$ is a mesh node, the cluster-head will update $C_{m}\left(v_{x},:\right)=0$ and $C_{m}\left(:, v_{x}\right)=$ 0 . If the node returns or a new node enters the network, the cluster-head shall process presence announcements in the network that shall reflect who a node is able to reach and updates $C_{c}$ or $C_{m}$ accordingly. This ensures an accurate view of connectivity in the network; and,

- Update Path Matrix: For a lost link, a cluster-head will find all instances of a link in the path matrix $X$ and de-activate all paths which contain the lost link. For a lost node, a cluster-head will de-activate all paths which contain the node such that the cross-layer optimization algorithm is only accessing valid paths. If the node returns, the cluster-head re-activates the path entries.

Both of these approaches enable the cluster-head's knowledge of the state of the network to remain up-to-date. Signaling protocols will be covered in Chapter 5 .

\subsection{Data Link Layer}

\subsubsection{Channel Optimization State}

It is the function of the link layer in the channel optimization state to determine the optimal channel allocation for a path. To perform this function, the link layer 
retrieves the subset path set $A \subset X$ for a given source sensor $v_{2}$. The link layer will also retrieve the link utility values $L_{v_{x}, v_{y}, f}^{*}$ computed at the PHY layer for each hop along these paths. Most importantly, however, is the fact that these link utility values have been computed over all channel bins $f \in F$. These bins, each with bandwidth $w_{f}$, are now evaluated at the link layer to find the optimal valid channel allocation subject to the objective function constraints.

Consider a sample table of optimal link utilities in Table 4.1. These values are presented for all bins in the spectrum band for a single candidate path, path ${ }_{5,104}$ $=\left\{\begin{array}{lllll}5 & 8 & 64 & 101 & 104\end{array}\right\}$, originating from our UWB cluster. The cluster for which the table applies has $V=101$ nodes (formed of 100 sensors and the cluster-head). As used previously, the master node also has actual mesh ID 3 ; recall that after forming $C_{\text {merged }}$, the master node was assigned temporary ID 4 with a $C_{m e r g e d}$ index of $V+4-1=104$. Also, in Table 4.1, 15 UWB channels are used.

Table 4.1: Optimized Link Utilities $L^{*}$ for UWB Channels for Candidate Simple Path

\begin{tabular}{ccccccccccccc}
\hline \hline Hop Number & Node ID & \multicolumn{10}{c}{$L^{*}$ in bin number } \\
\hline & & 1 & 2 & 3 & 4 & 5 & 6 & 7 & 8 & 9 & 10 & 15 \\
\hline 1 & 5 & 659 & 2955 & 4072 & 2568 & 5666 & 4341 & 2043 & 812 & 5387 & 1415 & 5220 \\
\hline 2 & 8 & 3824 & 6284 & 196 & 2843 & 1345 & 4761 & 4170 & 4381 & 1158 & 55 & 2330 \\
\hline 3 & 64 & 3786 & 1185 & 4175 & 552 & 615 & 4102 & 3653 & 4422 & 6170 & 1954 & 165 \\
\hline 4 & 101 & 3392 & 3636 & 5448 & 3779 & 1222 & 3043 & 4314 & 6046 & 1298 & 1784 & 1421 \\
\hline Destination & 104 & - & - & - & - & - & - & - & - & - & - & - \\
\hline
\end{tabular}

Upon generating the link utilities above, the algorithm ranks the $C$ most preferred bins for each hop $v_{x}-v_{y}$ in the order of the link utility $L_{v_{x}, v_{y}, f}^{*}$ gained. The result is the preferred bin table in Table 4.2 for which $C=5$. This table gives us the order of channels that the transmitter-receiver pairs at each hop prefer. 
Table 4.2: Preferred Channels in Order of Optimized Link Utility $L^{*}$

\begin{tabular}{ccccccc}
\hline \hline Hop Number & Node ID & \multicolumn{5}{c}{ Preference Order } \\
\hline & & $1^{\text {st }}$ & $2^{\text {nd }}$ & $3^{\text {rd }}$ & $4^{\text {th }}$ & $5^{\text {th }}$ \\
\hline 1 & 5 & 5 & 9 & 15 & 6 & 3 \\
\hline 2 & 8 & 2 & 6 & 8 & 7 & 1 \\
\hline 3 & 64 & 9 & 8 & 3 & 6 & 1 \\
\hline 4 & 101 & 8 & 3 & 7 & 4 & 2 \\
\hline Destination & 104 & - & - & - & - & - \\
\hline
\end{tabular}

For these preference rankings, it is possible for all transmitter-receiver pairs to utilize their most preferred channels, as they satisfy the objective function constraints, namely that successive nodes in a path do not send over the same channel which would create interference for a pipeline transmission of packets along a path; a description of the objective function constraints was provided in Section 3.7.2. Thus, the preferred bin allocation is selected as $f_{1}^{*}, \ldots, f_{K^{\prime}}^{*}=\left\{\begin{array}{lll}52 & 9 & 8\end{array}\right\}$ for this candidate path.

In some cases, utilizing the most preferred bin allocation will not be possible. In observing a different preferred bin allocation in Table 4.3 (for which $L_{\boldsymbol{v}_{\boldsymbol{x}}, v_{y}, f}^{*}$ values have not been provided), not all first preferences can be satisfied. In this case, both sensors with IDs 5 and 8 prefer to use $f_{1}=f_{2}=5$ although the objective function

Table 4.3: Preferred Channels Where First Preference is Not Valid

\begin{tabular}{ccccccc}
\hline \hline Hop Number & Node ID & \multicolumn{5}{c}{ Preference Order } \\
\hline 1 & & $1^{\text {st }}$ & $2^{\text {nd }}$ & $3^{\text {rd }}$ & $4^{\text {th }}$ & $5^{\text {th }}$ \\
\hline 2 & 5 & 5 & 1 & 13 & 7 & 3 \\
\hline 3 & 8 & 5 & 4 & 6 & 12 & 1 \\
\hline 4 & 64 & 7 & 14 & 3 & 6 & 10 \\
\hline Destination & 101 & 8 & 3 & 4 & 9 & 1 \\
\hline
\end{tabular}


constraints prohibit it. Thus, based on the corresponding $L_{v_{x}, v_{y}, f}^{*}$ values across these preferred bins, it is necessary to find a valid combination that leads to the largest valid profit sum, $U_{v_{2}, v_{j}}^{a^{*}}$.

This is performed by analyzing $C^{K^{\prime}}$ permutations of preferred bins to determine the valid allocation that has the highest $U_{v_{v}, v_{j}}^{a}$. The algorithm starts with each hop's most preferred bin, i.e. $\{5578\}$, and ends with each hop's $C^{\text {th }}$ least preferred bin, i.e. $\left\{\begin{array}{llll}3 & 1 & 10 & 1\end{array}\right\}$, with all combinations in between. The valid bin allocation with the highest $U_{v_{\imath}, v_{j}}^{a}$ and satisfies our objective function constraints is selected as the channel allocation with the greatest suitability for this candidate path. The optimal channel allocation is denoted as $f_{1}^{*}, \ldots, f_{K^{\prime}}^{*}$

\subsection{PHY Layer}

\subsubsection{Link Optimization State}

The negotiation for PHY layer parameters in the Link Optimization State is the basis

of our resource negotiation. By optimizing the link utility $L_{v_{x}, v_{y}, f}^{*}$ in (4.9) over all hops along a candidate path, we can determine the suitability of a link over all channels for a transmission request. The link utility function provided the optimization equation for the link utility $L^{*}$, for which the optimal rate $r_{v_{x}, v_{y}, f}^{*}$ is calculated according to

$$
L_{v_{x}, v_{y}, f}^{*}\left(r_{v_{x}, v_{y}, f}^{*}, t\right)=\alpha \log _{10}\left(1+\frac{r_{v_{x}, v_{y}, f}^{*}}{C_{v_{y}, f}(t)}\right)-\psi(E) \frac{q_{t_{v_{x}}} l r_{v_{x}, v_{y}, f}^{*}}{m}+\eta
$$


(3.7). The rate $r_{v_{x}, v_{y}, f}^{*}$ corresponds to the transmission rate that shall be used on a link if it is ultimately selected in the final path.

Upon optimizing the link utility $L_{v_{x}, v_{y}, f}^{*}$ as a function of the rate $r_{v_{x}, v_{y}, f}^{*}$, the cluster-head stores the transmit power $P_{v_{x}, f}^{*}$ and rate $r_{v_{x}, v_{y}, f}^{*}$ that correspond to the optimal $L_{v_{x}, v_{y}, f}^{*}$ value for a particular link optimization. While this optimization is performed, it is very likely that certain $v_{x}-v_{y}$ hops along a path are repeated over several candidate paths. Thus, rather than optimizing these links again during the same resource negotiation, we maintain done_already $\left(v_{x}, v_{y}\right)$ for the $v_{x}-v_{y}$ pair during the service request. Prior to the link optimization, the cluster-head checks to ensure that $v_{x}-v_{y}$ has not been optimized already for another candidate path during this service request; if it has, i.e. done_already $\left(v_{x}, v_{y}\right)=1$, the cluster-head simply retrieves the $P_{v_{x}, f}^{*}, r_{v_{x}, v_{y}, f}^{*}$ and $L_{v_{x}, v_{y}, f}^{*}$ computed previously as the optimization performed for a hop occurs irrespective of the candidate path.

\subsection{Steps in Cross-Layer Optimization Process}

This section outlines the negotiation process to determine a resource allocation for a multi-hop path in our distributed WSN. The first step involves determining the list of available simple paths from sensors in both of our clusters to the master node. This is performed at the network layer while the cluster-heads are in the Initialization State using the SPP. It is not required that these paths are link-disjoint, but we require that each vertex in the graph is traversed at most once per path. 
Clusters periodically send data to the master node following a Poisson process of events with exponential inter-arrival times. Upon a request requiring cross-layer optimization, the second step of the negotiation involves enumerating over a set of candidate paths $A \subset X$ for a source node at the time of a request. In this enumeration, the link utilities for all hops across all paths are optimized to determine the optimal rate and transmit power for that hop over all possible channels; this is performed at the PHY layer according to the constraints in (3.6). Although many paths may include the same transmitter-receiver pair $v_{x}-v_{y}$, this link optimization over all channels only needs to be done the first time the link is encountered. This determines the optimal $L_{v_{x}, v_{y}, f}^{*}, r_{v_{x}, v_{y}, f}^{*}$ and $P_{v_{x}, f}^{*}$ for the hop. These parameters determine the link suitability at time $t$.

The third step involves determining the receiver's $C$ most preferred bins by forming Table 4.2 at the link layer. To do so, the receiver $v_{y}$ forms Table 4.1 and ranks the bins in order of the $L_{v_{x}, v_{y}, f}^{*}$ gained for $C$ channels. If the most preferred channels at each hop cannot be satisfied, following this, the link layer iterates over $C^{K^{\prime}}$ possible bin combinations to determine the valid combination that leads to the maximum valid profit average, $U_{v_{\imath}, v_{j}}^{a}$. In this fashion, we find the valid bin allocation with the greatest preference over all hops along a path.

The second-last step involves selecting the single candidate path that has been optimized that has the greatest average profit across all links in the path. The corresponding bin allocation $f_{1}^{*}, \ldots, f_{K^{\prime}}^{*}$ is selected and the suitability coefficient of the selected path is denoted as $U_{v_{\imath}, v_{j}}^{a^{*}}$. 
The final step involves informing the sensors along the path of their required channel, transmit power, data rate and next hop, which will be covered in Chapter 5. A summary of the resource negotiation using the cross-layer optimization policy is provided in Figure A.1 in Appendix A.

\subsection{Summary}

This chapter presented our cross-layer design and protocol layer functions. These included the optimization of: i) the selection of the optimal path at the network layer; ii) the allocation of operating channels across multiple frequency bands at the link layer; and, iii) the optimal transmit power and rate while considering residual energy capacities of nodes along a candidate path. The basis of our algorithm is the evaluation of the throughput benefits and power costs in terms of common PHY layer resources that impact both performance and energy metrics. A summary of the resource negotiation using the cross-layer optimization policy is provided in Figure A.1 in Appendix A. The next chapter will cover our system design, implementation strategies, simulation parameters and performance metrics. 


\section{Chapter 5}

\section{System Design}

This chapter focuses on implementation issues of the distributed WSN including a discussion on the deployment of redundant mesh nodes and the required information by cluster-heads for cross-layer optimization in the form of node state information. Following this discussion, the packet formats and NPP will be presented; network planning enables us to balance the requirements of application performance and network lifetime for next-generation WSNs, and is the third major contribution of this thesis. A discussion of system design issues will follow. These design issues surround our simulation methodology, the operating parameters of our optimization algorithm and the queueing model used in our study to service requests. The chapter will conclude by presenting the performance metrics used to evaluate our optimization algorithm. These performance metrics include measurements of the throughput benefits achieved by our cross-layer optimization algorithm, the achieved network lifetime and average waiting times for priority-based clusters, among others. 


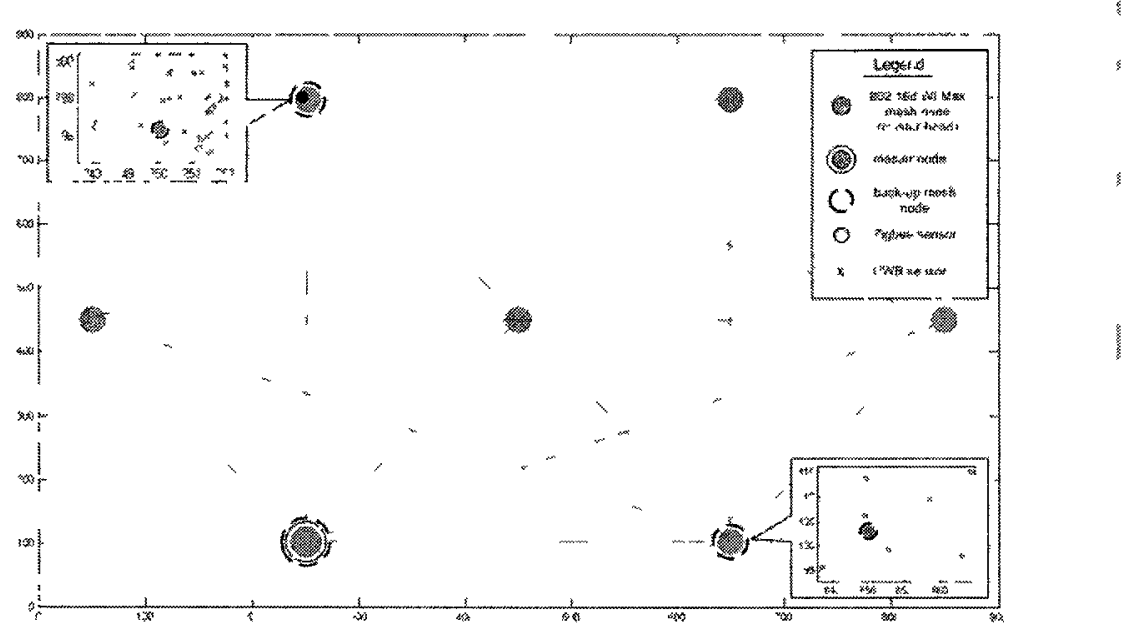

Figure 5.1: Back-up Cluster-Heads and Master Nodes in Distributed WSN

\subsection{Mesh Network Deployment}

In the network simulated in this work, cluster-heads are the single point of failure in a path from a source sensor to the master node. In the deployment of these networks, it will be important to equip the network with redundant cluster-heads and master nodes to address the survivability of our system. For master nodes, backup master nodes are activated when the main node's residual energy capacity falls below a certain level, or when the main node encounters a failure. Load-balancing can also be performed where nodes take regular turns being active, while the other remains dormant. Cluster-heads can follow a similar approach. Figure 5.1 presents this scenario with back-up mesh nodes.

Multiple cluster-heads servicing a single cluster can also be separated geographically within the cluster. In this manner, the same sensors are not repeatedly used 
in the routing of data to a cluster-head. A combined approach can also be utilized where cluster-heads are placed at separate locations but take turns being active.

The use of redundant mesh nodes raises additional research questions. Firstly, an optimal scheduling algorithm to activate back-up cluster-heads and master nodes should be explored. Secondly, the optimal placement of cluster-heads within a sensor cluster for maximum network lifctime, maximum coverage or minimal energy consumption should be addressed. Cluster-heads may be placed at strategic locations, as opposed to sensors that are typically dispersed randomly in a field (although sensors have been used in dynamic clustering algorithms to perform cluster-head functions as reviewed in Chapter 2). These two research problems are left for future work.

\subsection{Node State Information}

Given that the cluster-heads in our network perform centralized cross-layer optimization of sensor requests, the first step of the system design process is to define the state of a node $v_{x}$ at any time. This state information includes:

- Node identifier (SID): the ID of sensor or mesh node $v_{x}$;

- Sub-network identifier (SNID): the ID of the sub-network (cluster or mesh network) in which the node $v_{x}$ resides;

- Energy rating information (ERI): the remaining energy of node $v_{x}$ in the form of a percentage of the initial energy capacity, $e_{v_{x}} / E_{v_{x}} ;$ and, 
- Surrounding interference temperature (SIT): the measurcment of the surrounding interference plus noisc $(I+N)$ cnergy as measurcd by node $v_{x}$.

Statc information is broadcastcd to announce presence in the network and is also used in the propagation of resource allocation to nodes selected during the optimization process using a coordination channcl. The state information above and the position of a node are all that a cluster-head requires to optimize a request. Next, we will cxplore how state information is used in the exchange of coordination messages for announcing presence and relaying routing decisions in the network. These coordination protocols support centralized control of joint throughput and energy optimization and we have submitted them for publication in a tutorial article in [105].

\subsection{Message Formats}

\subsubsection{Hello Messages for Presence Broadcast}

Node state information is used in the formation of hello messages sent between mesh nodes, and also between sensor nodes and their cluster-heads to announce presence in the network. The exchange of hello messages maintains accurate connectivity tables at the cluster-head. Thesc hello messages also update the cluster-head's knowledge of the state of a sensor or mesh node $v_{x}$ in terms of remaining energy capacity.

The hello packet format is presented in Figure 5.2 where the LAT and LONG fields represent the latitude and longitude of the node, respectively, using GPS. The 


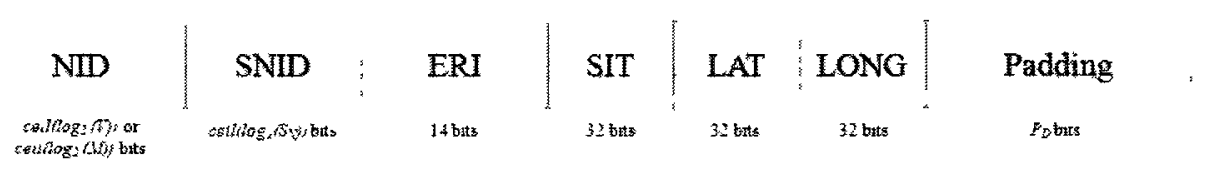

Figure 5.2: Hello Packet Format

NID field is used to identify the source of the hello packet and, hence, is the number of bits necessary to represent the node identifier. Thus, the NID field has a length of $\operatorname{ceil}\left(\log _{2}(V)\right)$ or ceil $\left(\log _{2}(M)\right)$ bits depending on if the sender is a sensor or mesh node. The SNID is the number of bits needed to identify the sub-network in which the node resides. The ERI field is 14 bits in fixed point number representation to represent the percentage of initial energy capacity remaining (using a scaling factor of $1 / 100$ ), i.e. sending 14 bits that represent 10,000 in decimal would deem an ERI of $100.00 \%$; we use fixed point number representation for the ERI field, as opposed to single precision floating point (32 bits long), because the ERI has a fixed number of digits - two - after the decimal point. This enables us to reduce the number of bits needed to represent the residual energy.

The SIT field, on the other hand, given real-time variations in the level of interference, is represented in full 32-bit single precision floating point format. Full 32-bit single precision floating point is also used to represent both the LAT and LONG fields. As nodes are stationary, it may only be necessary to include the LAT and LONG fields in the initialization phase to inform cluster-heads of node positions, after which it may not be required. Nevertheless, we include the LAT and LONG fields in the hello message, while $P_{D}$ bits of padding may be used to fill out the packet. 
For a distributed WSN of $S_{N}=3$ sub-networks (with two sensor clusters and the mesh network presented in Figure 3.1), $V=1,000$ sensor nodes per cluster and a mesh network composed of $M=7$ mesh devices, the hello packet has a length of 16 bytes. This includes ten or three bits for the NID field, and padding of six or thirteen bits, depending on if the source is a sensor or mesh node, respectively. The length of the hello packet is denoted as $m_{h}$ in bits.

We design one hello packet format for both the sensor and mesh network to simplify the system design process and to reduce decoding complexity. A packet length of 15 bytes could have been used in the mesh network, but we choose to have a common packet format at the expense of a transmitting an extra byte of padding. With that said, it may be necessary to design separate packet formats depending on the number of sensor and mesh nodes in the network and the overhead associated with using a single format.

\subsubsection{Routing Decision Updates}

Upon determining the optimized resource allocation for a request from a source sensor $v_{\imath}$, a cluster-head $v_{m}$ will propagate a routing decision update (RDU) to each node $v_{x} \in$ path $_{v_{\imath}, v_{j}}$. The RDU is sent backwards along the path in the sensor network to the source $v_{\imath}$, and forwards along the path in the mesh network to the master node $v_{j}$ as shown in Figure 5.3. This ensures that all nodes along the path are aware of their

necessary resource allocations. To do so, cluster-head $v_{m}$ will include the following 


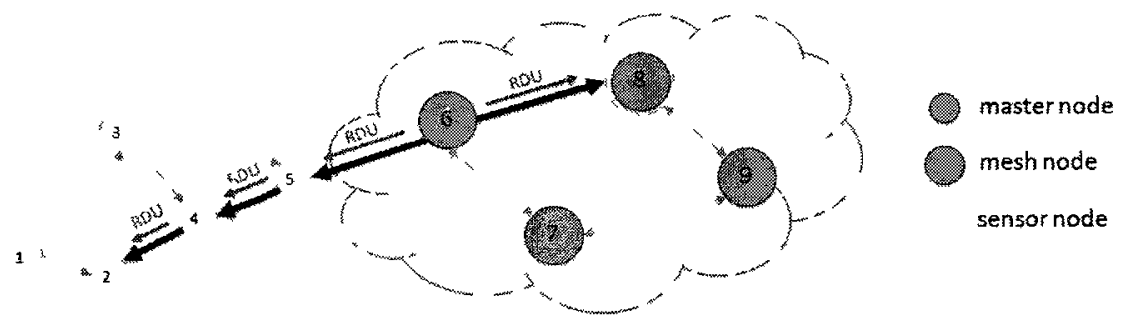

Figure 5.3: Propagation of RDU through Sensor and Mesh Networks

parameters such that all nodes can update their forwarding tables with up-to-date information:

- Sending sensor identifier (SSID): the ID of source sensor $v_{\imath}$ that is the origin of the request;

- Request identifier (RID): the ID of the request being serviced at source sensor $v_{\imath}$ given that sensors may support multiple requests;

- Previous-hop identifier (PHID): the ID of node $v_{x-1}$ from whom $v_{x}$ will receive packets; and,

- Next-hop identifier (NHID): the ID of node $v_{x+1}$ to whom $v_{x}$ will forward packets.

Figure 5.4 illustrates the format of the RDU message based on this information. In addition, the cluster-head will include the NID of each node along the determined path such that they can retrieve their necessary operating parameters when they receive the routing decision. The message will also include the transmit power POW (each hop in 32-bit single precision floating point format), and operating channel FRQ 


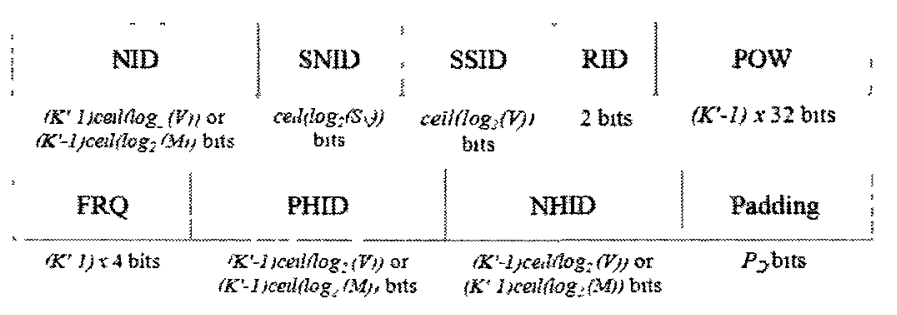

Figure 5.4: RDU Packet Format

(four bits to represent the channel number to be used per hop) determined during the optimization process, and the SNID of the sub-network in which the node resides. $P_{D}$ bits of padding may also be used.

The RDU packet format is designed such that each RDU contains information for at most $\left(K^{\prime}-1\right)$ hops, with separate RDUs being sent backwards through the sensor network to the source and forwards through the mesh network to the master node. Recall that, since the cluster-head acts as a bridge between the sensor cluster and the mesh network, at least one hop must reside in each sub-network. Hence, information for only $\left(K^{\prime}-1\right)$ hops is required.

Given the same conditions as for the hello packet of $S_{N}=3$ sub-networks, $V=$ 1, 000 sensor nodes and $M=7$ mesh nodes, we design our network with separate RDUs for the sensor and mesh network, as the overhead associated with a single format is significant; the mesh network would need to transmit over eight bytes of overhead per RDU packet for a single format. Thus, for the sensor and mesh networks, the RDU has a length of 27 bytes and 19 bytes, respectively. We denote the RDU packet lengths for the sensor and mesh networks as $m_{r}$ and $m_{r_{m}}$, respectively. 


\subsubsection{Data Packets}

In the data transmission process, upon receiving a data packet from the PHID, an intermediate node $v_{x} \epsilon$ path $h_{v_{2}, v}$ will set their next-hop based on the NHID provided by the cluster-head in the routing decision update. Data packets will be formed of the transmitting node's SID, originating sensor's SSID, RID, and the following:

- Payload information: the gathered sensory information for feedback; and,

- Transmission priority level (TPL): the priority of the transmission (high or low) for routing preference.

While transmissions from different clusters may be of different priorities, typically transmissions from a given sensor cluster all have the same priority at any given time. A discussion of priority schemes will be presented later in this chapter.

\subsection{Network Planning for Predictable Lifetimes}

Given the increased emphasis on application performance in next-generation networks, we must strive for peak performance while achieving predictable network lifetimes. An important step in this process is quantifying the relationship between: the expected performance given the communication protocol being used, the desired network lifetime, the energy capacities of nodes in the network, the number and sizes of data and coordination packets routed by a node, the expected holding time of a transmission, and the sleep energy dissipation. 
By considering the relationship between these factors, we are able to tie resource consumption to network operation such that we can truly understand the benefits and costs of various design decisions. We surmise that this relationship will enable us to make more informed decisions related to resource allocation by studying their impacts on both application performance and network lifetime. Determining the relationship between these parameters is also crucial to allowing network planners of next-generation WSNs to derive the necessary initial energy capacities, or correspondingly, the message loads that can be supported given a fixed energy capacity.

To calculate the required initial energy capacities for sensors and mesh nodes in our network, it is important to review the expected energy dissipations of transmitters and receivers from Chapter 2. We provide them here again for reference as,

$$
\begin{aligned}
E_{t_{x_{d s s}}}(l, n) & =\left(\frac{q_{t x} l E[R]}{m}\right)\left(\frac{m n}{E[R]}\right)=q_{t x} l n \\
& =\zeta q_{t_{v_{x}}} l n
\end{aligned}
$$

and,

$$
\begin{aligned}
E_{r_{x_{d r s s}}}(l, n) & =\left(\frac{q_{r x} l E[R]}{m}\right)\left(\frac{m n}{E[R]}\right)=q_{r x} l n \\
& =(1-\zeta) q_{t_{v_{x}}} l n
\end{aligned}
$$

We shall define that each mesh node has on average $E\left[N_{m}\right]$ neighbors where $E\left[N_{m}\right]=M p_{m}$; probability $p_{m}$ is the mean of a Bernoulli random variable that represents the probability of a successful connection in the mesh network. Conversely, each sensor has $E\left[N_{s}\right]=V p_{s}$ neighbors on average where $p_{s}$ is the mean probability of a successful connection in the sensor network. 


\subsubsection{Sensors}

In calculating the initial energy capacity for a sensor node, the required energy capacity $E_{v_{x}}$ to sustain the sensor node can be calculated as,

$$
E_{v_{x}}=E_{A W}+E_{A S}
$$

where $E_{A W}$ and $E_{A S}$ are the total energies consumed while a node is awake and asleep, respectively. $E_{A W}$ is derived as,

$$
E_{A W}=E_{A W_{d a t a}}+E_{A W_{c o}}
$$

where $E_{A W_{\text {data }}}$ is the sum of the energy expended in relaying sensory data as a relay node on behalf of another sensor and transmitting gathered sensory data as a source. We calculate $E_{A W_{\text {data }}}$ as follows,

$$
\begin{aligned}
& E_{A W_{\text {data }}}=E_{A W_{\text {relay }}}+E_{A W_{\text {transmatter }}} \\
& =\frac{T_{L}}{T_{M}}\left(T_{R}(V-1)\left(E_{r_{x_{d \imath s s}}}\left(l_{p}, n_{p}\right)+E_{t_{x_{d \imath s s}}}\left(l_{p}, n_{p}\right)\right)\right. \\
& \left.+E_{t_{x_{d s s}}}\left(l_{p}, n_{p}\right)\right)
\end{aligned}
$$

for a desired network lifetime $T_{L}$ and average time between sensor requests $T_{M}$, the ratio $T_{L} / T_{M}$ estimates the number of arriving requests per node. $V$ is the number of sensor nodes in the node set, and $l_{p}$ is the payload of the packet in bits. $T_{R}$ is the percentage of time a node acts as a relay node and can be computed from path 
matrix $X$, also defined in Chapter 3. By subbing (5.1) and (5.2) into (5.4), we can simplify $E_{A W_{d a t a}}$ as,

$$
E_{A W_{\text {data }}}=\frac{T_{L}}{T_{M}}\left(q_{t_{x}} l_{p} n_{p}\left(T_{R}(V-1)+\zeta\right)\right)
$$

$E_{A W_{c o}}$ is the energy dissipated by a node in contributing to coordination in the network through the handling of hello packets and RDU packets from its neighbors. $E_{A W_{c o}}$ considers the time between hello messages $T_{H}$, and the payloads of the hello packet $l_{h}$ and RDU packet $l_{r} . E_{A W_{c o}}$ also considers the amount of energy, $E\left[N_{s}\right] E_{r_{x_{d \imath s}}}\left(l_{h}, 1\right)$, dissipated by a node in receiving hello messages from $E\left[N_{s}\right]$ neighbors and the energy, $E_{t_{x_{d_{2} s s}}}\left(l_{h}, 1\right)$, the node dissipates in transmitting its own hello message. With this information, $E_{A W_{c o}}$ is calculated as,

$$
\begin{aligned}
E_{A W_{c o}}= & E_{A W_{\text {hello }}}+E_{A W_{R D U}} \\
=\frac{T_{L}}{T_{H}} & \left(E_{t_{x_{d i s s}}}\left(l_{h}, 1\right)+E\left[N_{s}\right] E_{r_{x_{d r s s}}}\left(l_{h}, 1\right)\right) \\
& +\frac{T_{L}}{T_{M}}\left((V-1) T_{R}\left(E_{t_{x_{d r s s}}}\left(l_{r}, 1\right)+E_{r_{x_{d r s s}}}\left(l_{r}, 1\right)\right)\right) \\
& +\frac{T_{L}}{T_{M}} E_{r_{x_{d r s s}}}\left(l_{r}, 1\right)
\end{aligned}
$$

where $T_{R}$ is the percentage of time a sensor is chosen as a relay node. The energy consumed due to the RDU is the sum of the energy a node uses in relaying an RDU as an intermediary node, and the energy necessary to receive the RDU as the source sensor of the transmission for each request. This plans for the worst case energy burden for network planning by assuming that no requests are rejected. 
We choose $T_{H}$ to be sufficiently large in sensor networks (i.e. two or three seconds) to reduce the number of coordination messages routed by energy-constrained sensors in the network. Given the predictable nature of our NPP, the cluster-head can use the elapsed time since the last presence announcement to estimate the current state of the remaining energy capacity of a sensor, if necessary. As a result, we can reduce the number of coordination messages required, while providing sufficient feedback to update state information at the cluster-head.

By subbing (5.1) and (5.2) into (5.6), we get,

$$
E_{A W_{c o}}=q_{t_{v_{x}}} T_{L}\left(\frac{l_{r}}{T_{M}}\left((V-1) T_{R}+(1-\zeta)\right)+\frac{l_{h}}{T_{H}}\left(\zeta+(1-\zeta) E\left[N_{s}\right]\right)\right)
$$

The only term left to derive for the sensor node is the sleep energy $E_{A S}$, which is,

$$
E_{A S}=V_{T} I_{A} T_{a s l e e p}
$$

as the amount of energy the node expends while it is asleep, where $V_{T}$ is the voltage rating of the node's battery and $I_{A}$ is the sleep current. $T_{\text {asleep }}$, the amount of time that the node is asleep, is determined as,

$$
T_{\text {asleep }}=T_{L}-\left(T_{A W_{\text {data }}}+T_{A W_{\text {hello }}}+T_{A W_{R D U}}\right)
$$

where $T_{A W_{\text {data }}}, T_{A W_{\text {hello }}}$ and $T_{A W_{R D U}}$ are the durations that a node is awake due to data transmission, and handling hello and RDU messages, respectively. 


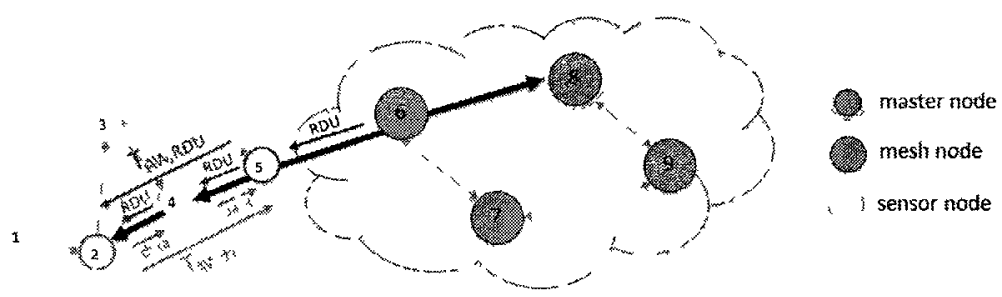

Figure 5.5: Duration Node Remains Awake Upon Receiving RDU, $T_{A W_{R D U}}+T_{A W_{d a t a}}$

If a node is chosen as a relay node by the cluster-head during the optimization process, as illustrated in Figure 5.5, upon receiving the RDU, the given sensor will remain awake until it receives the data packets from its PHID and forwards them to its NHID. This is crucial in sensor networks for implementing survivability. If the node enters sleep mode after transmitting the RDU, it will be unaware if a timeout has occurred. If after a timeout value chosen to be a maximum of one second (to give enough time for data packets but not excessive delay if information is time-sensitive), data has not been received, a relay node awaiting data will send a lost link signal to its NHID to be propagated forward along the path to the cluster-head. This ensures a high level of survivability in our network and enables the cluster-head to re-optimize the path by ignoring the lost link in the optimization.

This is even more crucial in sensor networks that use data correlation to transmit a single measurement to represent a number of correlated sensor readings [50]; the cluster-head must be made aware of the failure in the transmission to re-optimize the path. Thus, a selected relay node will remain awake for $T_{A W_{d a t a}}+T_{A W_{R D U}}$, which is not very long given our design decision of a maximum number of hops, $K^{\prime}=4$. 


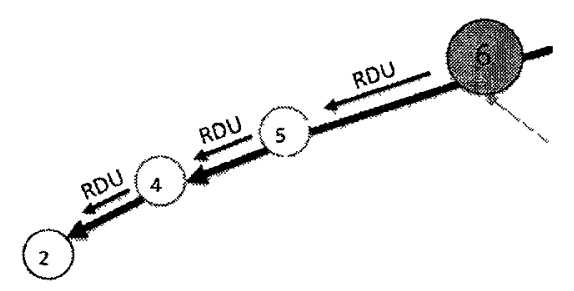

Figure 5.6: Relay Nodes in a Sample Path in the Sensor Cluster

The duration of time, $T_{A W_{R D U}}$, that the node remains awake to send and receive RDU messages will subsequently calculate the average waiting time of selected relay nodes based on their position in the path. Consider the path [2 $\left.4 \begin{array}{lll}2 & 5 & 6\end{array}\right]$ in Figure 5.5 for which $K^{\prime}=4$ and node 8 is the master node. There are at most $\left(K^{\prime}-2\right)$ possible relay positions in the sensor network considering that sensor relay nodes also cannot include the cluster-head (node 6). These relay positions are nodes 4 and 5 , which are highlighted in Figure 5.6. In this example, node 5 will remain awake for a duration of $3 m_{r} / E[R]$ seconds. Node 4 , on the other hand, will remain awake for $2 m_{r} / E[R]$ seconds. This information is used to derive the average waiting time as a source sensor and a relay node as,

$$
T_{A W_{R D U}}=\frac{T_{L}}{T_{M}}\left((V-1) T_{R}\left(\frac{m_{r}}{E[R]\left(K^{\prime}-2\right)} \sum_{k=2}^{K^{\prime}-1} k\right)+\frac{m_{r}}{E[R]}\right)
$$

This solution is valid for all $K^{\prime} \geq 3$. For $K^{\prime}=2$, no time is spent as a sensor relay node and $T_{A W_{R D U}}=\left(T_{L} / T_{M}\right)\left(m_{r} / E[R]\right)$ only.

$T_{A W_{\text {data }}}$, the duration of time the node spends in sending and receiving data packets is shown below. Again, the series represents the average time that it takes for a node 

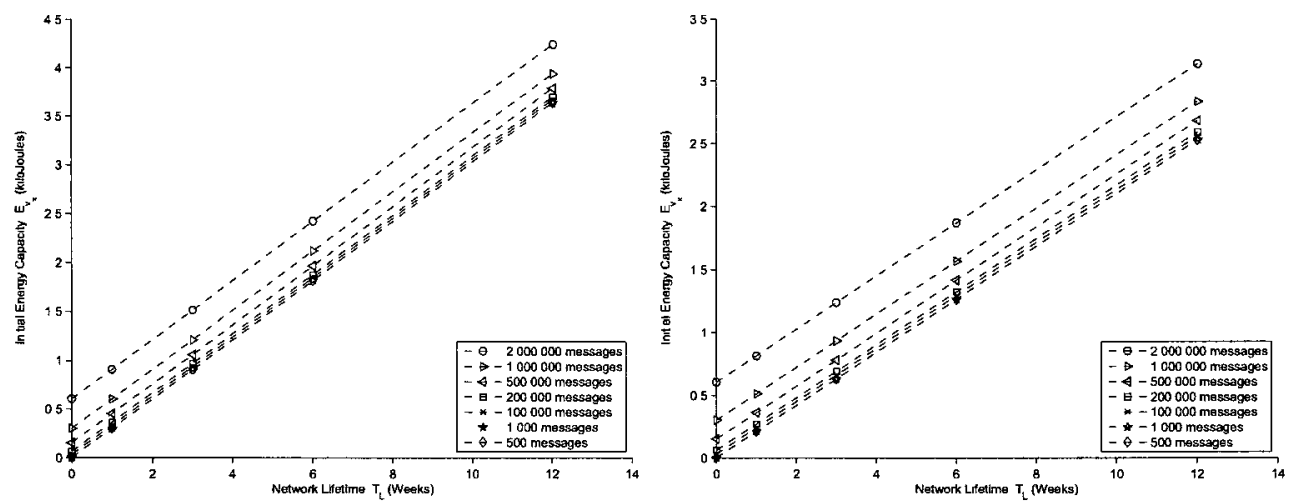

(a) Time between Presence Announcements, (b) Time between Presence Announcements, $T_{H}=3$ seconds

$T_{H}=5$ seconds

Figure 5.7: Energy Capacities vs. Desired Network Lifetime for Various UWB Sensor Message Loads

to relay packets. The difference here is that the pipelining data transmission of $k$ stages has $n_{p}$ packets to relay, each of size $m_{p}$ bits.

$$
T_{A W_{\text {data }}}=\frac{T_{L}}{T_{M}}\left((V-1) T_{R}\left(\frac{m_{p}}{E[R]\left(K^{\prime}-2\right)} \sum_{k=1}^{K^{\prime}-2}\left(n_{p}+k\right)\right)+\frac{m_{p} n_{p}}{E[R]}\right)
$$

$T_{A W_{\text {hello }}}$ can be derived to measure the overall time for a node to receive $E\left[N_{s}\right]$ hello packets and announce its own presence where,

$$
T_{A W_{\text {hello }}}=\frac{T_{L}}{T_{H}}\left(\frac{m_{h}}{E[R]}+E\left[N_{s}\right] \frac{m_{h}}{E[R]}\right)
$$

The relationship between the initial energy capacity $E_{v_{x}}$ and the desired network lifetime $T_{L}$ is shown in Figure 5.7 for an UWB sensor for two durations between presence announcements, $T_{H}=3$ seconds and $T_{H}=5$ seconds. In this example, 
$\zeta=0.5, V_{T}=2.5$ volts and $I_{A}=11 \mu \mathrm{A}$. For the sensor network parameters: $V=$ 1,000 nodes, $T_{R}=5 \%, m_{p}=38$ bytes, $l_{p}=32$ bytes, $n_{p}=4$ packets and $E[R]$ is 480 Mbps from Table 1.2. Also, recall for coordination packets that $m_{h}=16$ bytes and $m_{r}=27$ bytes, where $m_{h}$ and $m_{r}$ have six bits and four bits of padding, respectively.

Figure 5.7 leads to two significant conclusions. Firstly, for say 1,000 messages by an UWB sensor over a one year network lifetime (note that a message equates to the transmission of sensory data in response to a request), we require $3.4 k J$ and 2.4 $k J$ for $T_{H}=3$ seconds and $T_{H}=5$ seconds, respectively; these initial capacities are equivalent to under a watt of power per sensor, specifically $0.94 \mathrm{~W}$ and $0.67 \mathrm{~W}$ for the two cases. These messages amount to one million measurements received from all sensors at the master node over the network lifetime. These capacities provide a benchmark from which we may measure our requirements against available energy capacities in the market and scale our requirements as needed.

Secondly, the drop-off in the required initial capacities from $T_{H}=3$ and $T_{H}=5$ amounts to one kilojoule of energy, which is significant. Thus, care must be taken in the selection of coordination protocols during system design as they place a major energy burden on the network.

By modeling our energy capacity in terms of application performance, network lifetime $T_{L}$, and the message load through $T_{M}$ and $T_{H}$, we develop the NPP for sensors. We can use this information to tune the performance of our network based on a number of design considerations in planning our network. We can either design our network with necessary initial energy capacities to optimize application performance 
based on throughput, or correspondingly, determine the number and frequency of data and coordination messages that can be supported given a fixed energy capacity. In real-time, we may also evaluate the trade-offs inherent in various resource allocations to determine the effects on network lifetime and throughput performance; as used in this thesis, throughput is a function of physical resource consumption according to the Shannon rate $R$ that was discussed in our problem formulation in Chapter 3. Thus, we can make critical resource allocation decisions based on our cross-layer optimization algorithm for next-generation WSNs.

\subsubsection{Cluster-Heads}

The initial energy capacities for a cluster-head differ from that of sensors because the cluster-head is a mesh node that is responsible for relaying sensor packets from a cluster to the mesh network, as well as packets between mesh nodes. Thus, the clusterhead uses different technologies to communicate with its subordinate sensors and its mesh network peers. As a result, software radio will be critical in next-generation networks to convert the parameters between the sensor and mesh communication protocols at the cluster-head.

Consider the distributed WSN with two sensor clusters as depicted in Figure 5.1. A cluster-head will act as a bridge between the mesh network and, for example, the UWB sensor cluster (denoted as cluster 1 ). The cluster-head is also a potential relay node for transmissions arriving to the mesh network from the Zigbee cluster (denoted 
as cluster 2). Thus, the energy dissipation for a cluster-head will differ based on the source cluster of the transmission. In this section, we will derive the relationships for the cluster-head from the perspective of the UWB cluster.

When the cluster-head is acting on behalf of its sensor cloud, the energy dissipations are,

$$
E_{C H_{r c_{d s s}}}\left(l_{1}, n_{1}\right)=(1-\zeta) q_{t_{1}} l_{1} n_{1}
$$

and,

$$
E_{C H_{t c_{d r s s}}}\left(l_{m}, n_{m}\right)=\zeta q_{t_{m}} l_{m} n_{m}
$$

when receiving packets from the sensor cluster and transmitting those packets to the mesh network, respectively. In the above equations, $q_{t_{1}}, l_{1}$ and $n_{1}$ are the energy consumption per payload bit, the payload size and the number of packets used in the UWB sensor cluster, respectively. The terms $q_{t_{m}}, l_{m}$ and $n_{m}$ are the energy consumption per payload bit, the payload size and the number of packets used in the mesh network. The mesh network uses $n_{m}$ packets in case segmentation of packets is required. When acting as the cluster-head in routing packets from the sensor network to the mesh network, the energy dissipation is,

$$
E_{C H_{d 2 s s}}\left(l_{1}, l_{m}, n_{1}, n_{m}\right)=E_{C H_{r c_{d r s s}}}\left(l_{1}, n_{1}\right)+E_{C H_{t c_{d \imath s}}}\left(l_{m}, n_{m}\right)
$$

As a mesh relay node, the energy dissipations for the cluster-head as a transmitter and receiver are different as the energy consumption per bit metric for the mesh and 
sensor technologies differ. Thus, the energy dissipation of the cluster-head as a mesh relay node can be calculated as,

$$
E_{C H_{r m_{d r s s}}}\left(l_{m}, n_{m}\right)=(1-\zeta) q_{t_{m}} l_{m} n_{m}
$$

and,

$$
E_{C H_{t m_{d} s s}}\left(l_{m}, n_{m}\right)=\zeta q_{t_{m}} l_{m} n_{m}
$$

where $n_{m}$ packets are relayed within the mesh network. Note that cluster-heads do not relay traffic between mesh nodes that originates from the sensor cluster they govern as this would yield routing loops. Thus, only the mesh protocol is used and only $q_{t_{m}}$ is present in (5.16) and (5.17). When operating as a mesh relay node for packets originating from the Zigbee cluster, the UWB cluster-head's energy dissipation is derived as,

$$
E_{m e s h_{d 2 s s}}\left(l_{m}, n_{m}\right)=E_{C H_{r m_{d r s s}}}\left(l_{m}, n_{m}\right)+E_{C H_{t m_{d 2 s s}}}\left(l_{m}, n_{m}\right)=q_{t_{m}} l_{m} n_{m}
$$

If $n_{2}$ packets are sent to the mesh network from the Zigbee cluster, $n_{m}$ packets are transmitted in the mesh network after segmentation, if required.

The required initial energy capacities for the cluster-head are calculated as,

$$
E_{C H_{1}}=E_{A W_{C H_{1}}}+E_{A S_{C H_{1}}}
$$


where $E_{A W_{C H_{1}}}$ is derived as,

$$
E_{A W_{C H_{1}}}=E_{A W_{C H_{1} \text { data }}}+E_{A W_{C H_{1 h e l l o}}}+E_{A W_{C H_{1 R D U}}}
$$

where,

$$
\begin{aligned}
E_{A W_{C H_{1} \text { data }}=} & \frac{T_{L}}{T_{M_{1}}} V_{1} E_{C H_{\text {drss }}}\left(l_{1}, l_{m}, n_{1}, n_{m}\right)+\frac{T_{L} T_{R_{2}} V_{2}}{T_{M_{2}}} E_{m e s h_{d 2 s s}}\left(l_{m}, n_{m}\right), \\
= & T_{L}\left(q_{t_{m}} l_{m} n_{m}\left(\frac{V_{1}}{T_{M_{1}}} \zeta+\frac{T_{R_{2}}}{T_{M_{2}}} V_{2}\right)+q_{t_{1}} l_{1} n_{1}\left(\frac{V_{1}}{T_{M_{1}}}(1-\zeta)\right)\right), \\
E_{A W_{C H_{1 h e l l o}}=} & \frac{T_{L}}{T_{H}}\left(E\left[N_{s}\right] E_{C H_{r c_{d r s s}}}\left(l_{h}, 1\right)+E\left[N_{m}\right] E_{C H_{r m_{d r s s}}}\left(l_{h}, 1\right)\right. \\
& \left.\quad+E_{C H_{t m_{\text {drss }}}}\left(l_{h}, 1\right)\right) \\
= & \frac{T_{L}}{T_{H}} l_{h}\left(\zeta q_{t_{m}}+(1-\zeta)\left(E\left[N_{s}\right] q_{t_{1}}+E\left[N_{m}\right] q_{t_{m}}\right)\right)
\end{aligned}
$$

and,

$$
\begin{aligned}
E_{A W_{C H_{1} R D U}=}=\frac{T_{L}}{T_{M_{1}}} V_{1}\left(\zeta q_{t_{1}} l_{r}+E_{C H_{t m_{d s s}}}\left(l_{r_{m}}, 1\right)\right) \\
\quad+\frac{T_{L} T_{R_{2}} V_{2}}{T_{M_{2}}} E_{\text {mesh }_{d z s s}}\left(l_{r_{m}}, 1\right) \\
=T_{L}\left(\frac{V_{1}}{T_{M_{1}}} \zeta\left(q_{t_{1}} l_{r}+q_{t_{m}} l_{r_{m}}\right)+\frac{T_{R_{2}} V_{2}}{T_{M_{2}}} q_{t_{m}} l_{r_{m}}\right)
\end{aligned}
$$

$E_{A S_{C H_{1}}}$, the energy consumed while the UWB cluster-head is asleep, is derived as,

$$
E_{A S_{C H_{1}}}=I_{A} V_{T}\left(T_{L}-\left(T_{A W_{C H_{1} \text { data }}}+T_{A W_{C H_{1 \text { hello }}}}+T_{A W_{C H_{1 R D U}}}\right)\right)
$$

where $T_{A W_{C H_{1} \text { data }}}$ is the time spent by the cluster-head in routing data, both in transferring packets from the sensor cluster towards the master node, and in relaying packets originating from the Zigbee cluster. $T_{A W_{C H_{1 \text { hello }}}}$ and $T_{A W_{C H_{1} \text { RDU }}}$ are the durations 
that the cluster-head spends handling hello and RDU packets, respectively. Recall that pipelined transmissions are used in transmitting both RDU and data packets; the cluster-head will remain awake for the duration of sending the RDU to the source in the sensor cluster, until data is received from its PHID and relayed to its NHID in the mesh network. $T_{A W_{C H_{1} \text { data }}}, T_{A W_{C H_{1 h e l l o}}}$ and $T_{A W_{C H_{1 R D U}}}$ are derived below as,

$$
\begin{aligned}
& T_{A W_{C H_{1 \text { data }}}=} \frac{T_{L}}{T_{M_{1}}} V_{1}\left(\frac{m_{1}}{E\left[R_{1}\right]}\left(\left(K^{\prime}-1\right)+\left(n_{1}-1\right)\right)+\frac{m_{m} n_{m}}{E\left[R_{m}\right]}\right) \\
&+\frac{T_{L} T_{R_{2}}}{T_{M_{2}}} V_{2}\left(\frac{m_{m}}{E\left[R_{m}\right]\left(K^{\prime}-2\right)} \sum_{k=1}^{K^{\prime}-2}\left(n_{m}+k\right)\right), \\
& T_{A W_{C H_{1 \text { hello }}}=} \frac{T_{L}}{T_{H}}\left(E\left[N_{s}\right] \frac{m_{h}}{E\left[R_{1}\right]}+E\left[N_{m}\right] \frac{m_{h}}{E\left[R_{m}\right]}+\frac{m_{h}}{E\left[R_{m}\right]}\right) \\
&= \frac{T_{L}}{T_{H}} m_{h}\left(\frac{E\left[N_{s}\right]}{E\left[R_{1}\right]}+\frac{E\left[N_{m}\right]+1}{E\left[R_{m}\right]}\right)
\end{aligned}
$$

and,

$$
\begin{aligned}
T_{A W_{C H_{1} R D U}}= & \frac{T_{L}}{T_{M_{1}}} V_{1}\left(\frac{m_{r}}{E\left[R_{1}\right]}\left(K^{\prime}-1\right)+\frac{m_{r_{m}}}{E\left[R_{m}\right]}\right) \\
& +\frac{T_{L}}{T_{M_{2}}} T_{R_{2}} V_{2}\left(\frac{m_{r_{m}}}{E\left[R_{m}\right]\left(K^{\prime}-2\right)} \sum_{k=2}^{K^{\prime}-1} k\right)
\end{aligned}
$$

\subsubsection{Mesh Relay Nodes}

Mesh relay nodes are selected to carry UWB and Zigbee cluster traffic $T_{R_{1}}$ and $T_{R_{2}}$ percent of the time, respectively. The energy capacity $E_{m e s h}$ is derived similarly as,

$$
E_{m e s h}=E_{A W_{m e s h}}+E_{A S_{m e s h}}
$$


where the relationship between the energy capacity and message load for data, hello and RDU packets is,

$$
E_{A W_{\text {mesh }}}=E_{A W_{\text {mesh }}^{\text {data }}}+E_{A W_{\text {mesh hello }}}+E_{A W_{\text {mesh }} \text { RDU }}
$$

where,

$$
\begin{aligned}
E_{A W_{\text {mesh }_{\text {data }}}=} & \frac{T_{L}}{T_{M_{1}}} V_{1} T_{R_{1}} E_{\text {mesh }_{\text {drss }}}\left(l_{m}, n_{m_{1}}\right) \\
& +\frac{T_{L}}{T_{M_{2}}} V_{2} T_{R_{2}} E_{\text {mesh }_{\text {drss }}}\left(l_{m}, n_{m_{2}}\right) \\
= & \left(\frac{T_{L}}{T_{M_{1}}} V_{1} T_{R_{1}}\right) q_{t_{m}} l_{m} n_{m_{1}}+\left(\frac{T_{L}}{T_{M_{2}}} V_{2} T_{R_{2}}\right) q_{t_{m}} l_{m} n_{m_{2}} \\
E_{A W_{\text {mesh }} \text { hello }}= & \frac{T_{L}}{T_{H}}\left(E\left[N_{m}\right] E_{C H_{r m_{d 2 s s}}}\left(l_{h}, 1\right)+E_{C H_{t m_{d 2 s s}}}\left(l_{h}, 1\right)\right) \\
= & \frac{T_{L}}{T_{H}} q_{t_{m}} l_{h}\left(E\left[N_{m}\right](1-\zeta)+\zeta\right)
\end{aligned}
$$

and,

$$
\begin{aligned}
E_{A W_{m e s h} R D U}= & \frac{T_{L}}{T_{M_{1}}} V_{1} T_{R_{1}} E_{m e s h_{d 2 s s}}\left(l_{r_{m}}, 1\right) \\
& +\frac{T_{L}}{T_{M_{2}}} V_{2} T_{R_{2}} E_{m e s h_{d 2 s s}}\left(l_{r_{m}}, 1\right) \\
= & \left(\frac{T_{L}}{T_{M_{1}}} V_{1} T_{R_{1}}+\frac{T_{L}}{T_{M_{2}}} V_{2} T_{R_{2}}\right) q_{t_{m}} l_{r_{m}}
\end{aligned}
$$

Here, mesh nodes will relay $n_{m_{1}}$ or $n_{m_{2}}$ data packets to differentiate between the number of packets arriving from the UWB and Zigbee clusters, respectively.

The duration of time the mesh node is asleep, $E_{A S_{m e s h}}$, is determined as,

$$
E_{A S_{\text {mesh }}}=I_{A} V_{T}\left(T_{L}-\left(T_{A W_{m_{e s h} h_{\text {data }}}}+T_{A W_{\text {mesh }_{\text {hello }}}}+T_{A W_{\text {mesh }} \text { RDU }}\right)\right.
$$


where the durations $T_{A W_{\text {mesh }} \text { data }}, T_{A W_{\text {mesh }} \text { hello }}$ and $T_{A W_{\text {mesh }} \text { RDU }}$ arc found as follows,

$$
\begin{aligned}
& T_{A W_{\text {mesh dala }}}=\frac{T_{L}}{T_{M_{1}}} V_{1} T_{R_{1}} \frac{m_{m}}{E\left[R_{m}\right]\left(K^{\prime}-2\right)} \sum_{k=1}^{K^{\prime}-2}\left(n_{m_{1}}+k\right) \\
& +\frac{T_{L}}{T_{M_{2}}} V_{2} T_{R_{2}} \frac{m_{m}}{E\left[R_{m}\right]\left(K^{\prime}-2\right)} \sum_{k=1}^{K^{\prime}-2}\left(n_{m_{2}}+k\right) \\
& T_{A W_{\text {mesh }} \text { hello }}=\frac{T_{L}}{T_{H}}\left(E\left[N_{m}\right] \frac{m_{h}}{E\left[R_{m}\right]}+\frac{m_{h}}{E\left[R_{m}\right]}\right) \\
& =\frac{T_{L}}{T_{H}} \frac{E\left[N_{m}\right]+1}{E\left[R_{m}\right]} m_{h},
\end{aligned}
$$

and,

$$
T_{A W_{\text {mesh } R D U}}=\left(\frac{T_{L}}{T_{M_{1}}} V_{1} T_{R_{1}}+\frac{T_{L}}{T_{M_{2}}} V_{2} T_{R_{2}}\right)\left(\frac{m_{r_{m}}}{E\left[R_{m}\right]\left(K^{\prime}-2\right)} \sum_{k=2}^{K^{\prime}-1} k\right)
$$

By forming the relationships between energy capacity, network lifetime and application throughput for sensors, clustcr-heads and mesh relay nodes, we quantify the trade-off between these factors for the NPP. We can also optimize both the sensor and mesh portions of a path to gain the mutual benefits of application performance and a sustainable network lifetime. We have submitted the results of the NPP for publication in [106] and evaluate its performance in Chapter 6.

\subsection{Queueing Model}

We consider that cach sensor acts as an individual $M / M / 3 / 3$ queueing system with $c=3$ servers and no waiting room. Although each cluster is supported with $F_{1}$ or 
$F_{2}>c$ sub-channels, we impose this server limit of $c=3$ to provide ample channels for relay traffic. As mentioned in Chapter 3 , the same channel is not used over multiple hops for a single transmission as nodes have different channel preferences. Requests arrive according to a Poisson process with arrival rate $\lambda$, while requests are serviced at each sensor node individually at a service rate of $\mu$. The transition rate diagram for the $M / M / c / c$ queueing system is presented in Figure 5.8.

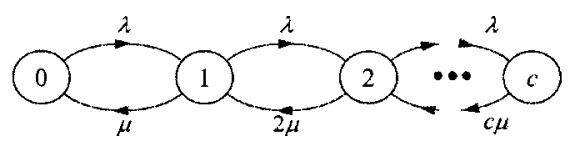

Figure 5.8: State Transition Diagram For $M / M / c / c$ Queueing System

A major consideration in the selection of the arrival rates $\lambda_{1}$ and $\lambda_{2}$ for our UWB and Zigbee clusters, respectively, is our desired network lifetime that imposes a strict bound on the network load. Increasing the network load, without consideration of the network lifetime that is set due to constrained sensor resources, will result in unexpected node outages. This illustrates the motivation behind our NPP to plan for a sustainable network lifetime while meeting the performance needs of next-generation sensory and resource management applications.

The network load is selected by setting a self-imposed limit of 1,000 requests per sensor node over the course of a one week network lifetime. This corresponds to arrival rates per sensor node of $\lambda_{s}=\lambda_{1}=\lambda_{2}=0.1$ requests $/$ minute. The cluster arrival rate of requests, $\lambda_{c}=\lambda_{n e t_{1}}=\lambda_{n e t_{2}}$, is 2 requests $/$ minute for each cluster for $V=20$ sensors. The network arrival rate of requests is $\lambda_{n e t}=\lambda_{\text {net }_{1}}+\lambda_{\text {net }_{2}}$. 
In computing the service time $1 / \mu$, we compute the expected holding times in a UWB and Zigbee transmission to find $\mu_{1}$ and $\mu_{2}$, respectively. By considering the path as a pipeline system with each relay node measuring the holding time for two stages (send and receive), we compute $1 / \mu_{1}$ and $1 / \mu_{2}$ as,

$$
\frac{1}{\mu_{1}}=\frac{m_{p_{1}}}{E\left[R_{1}\right]}\left(2+\left(n_{p_{1}}-1\right)\right)=\frac{m_{p_{1}}\left(n_{p_{1}}+1\right)}{E\left[R_{1}\right]}=3.04 \mu \mathrm{s}
$$

and,

$$
\frac{1}{\mu_{2}}=\frac{m_{p_{2}}}{E\left[R_{2}\right]}\left(2+\left(n_{p_{2}}-1\right)\right)=\frac{m_{p_{2}}\left(n_{p_{2}}+1\right)}{E\left[R_{2}\right]}=2.43 \mathrm{~ms}
$$

At any time, the expected number of transmissions in a sensor cluster can then be calculated as,

$$
E\left[N_{t}(t)\right]=\frac{\lambda_{c}}{\mu}\left(1-E\left[P_{B}(t)\right]\right)
$$

where $\mu$ is $\mu_{1}$ or $\mu_{2}$ depending on the sensor cluster of interest and $P_{B}(t)$ is the blocking probability in the cluster.

Generally, the Erlang B formula, which defines the probability that all $c=3$ servers are busy for a sensor, is used to compute the blocking probability but our system has the added constraint of multi-hop traffic which reduces the number of channels available to service a request. The Erlang B formula is,

$$
B\left(c, \rho_{s}\right)=p_{c}=\frac{\rho_{s}^{c} / c !}{1+\rho_{s}+\rho_{s}^{2} / 2 !+\ldots+\rho_{s}^{c} / c !}
$$


where,

$$
\rho_{s}=\frac{\lambda_{s}}{\mu}
$$

The determination of $E\left[P_{B}(t)\right]$ will be explored later in this chapter.

\subsection{Simulation Methodology}

As was discussed in Chapter 3, our distributed WSN is formed by a $V$-sized UWB sensor cluster and a $V$-sized Zigbee sensor cluster that are connected to a master node via an $M$-sized overlay mesh network. Our WSN spans a $1 \mathrm{~km} \times 1 \mathrm{~km}$ campus area for which $V=20$ sensor nodes (excluding the cluster-head) and $M=7$ mesh nodes; we simulate a small network size without loss of generality. As also mentioned in Chapter 3, our mesh nodes are organized in a hexagonal topology to achieve maximum coverage. The UWB cluster is focused in a $10 \mathrm{~m} \times 10 \mathrm{~m}$ area for a video feedback application, while the Zigbee cluster performs temperature sensing in a $75 \mathrm{~m} \mathrm{x} 75$ $m$ area such as a computer server room. The network energy capacities are chosen using our NPP with an expected network lifetime of one week.

\subsubsection{Optimization Variables}

In the optimization of our link utilities in (3.7) from Chapter 3 , our $\alpha, \beta, \gamma, \delta$ and $\eta$ parameters are selected per technology. They are selected through curve-fitting to shift the operating powers of the various technologies into the optimality range for increasing values of $E$ from (3.3). More specifically, the furthest pair of nodes 
within the maximum transmission distance for a communication protocol are taken and $\alpha, \beta, \gamma, \delta$ and $\eta$ are calibrated to ensure that the throughput selected during the optimization process adheres to the desired fraction of maximum throughput as a function of $E$. For example, only until minimal energy remains for a node should a node be unable to transmit at maximum throughput under optimal channel conditions. Changing the factors that impact the cross-layer optimization protocol will require a re-calibration of the operating parameters for $\alpha, \beta, \gamma, \delta$ and $\eta$ based on the process discussed above. Thesc factors includc: the technologies used in the network that affect the maximum transmission ranges of the nodes; the achievable transmission rates or link capacities; or, the factors that affect the path loss model such as the path loss exponent $n$ if, for example, outdoor sensory applications are desired.

A design decision was also made to ensure that $\alpha, \beta, \gamma, \delta$ and $\eta$ are tuned in such a manner that the $L^{*}$ values for hops within the mesh network are approximately double the $L^{*}$ values for hops in a sensor cluster. This provides an advantage to mesh network hops in the path selection process. In this fashion, we aim to steer the algorithm to prefer paths which favor routing through the mesh network to reduce the routing burden on sensors in the network. Variations in the $L^{*}$ valucs for hops within the cluster are still reflected in the average maximum profit $U_{v_{2}, v_{j}}^{a^{*}}$ when path

Table 5.1: Selcction of Optimization Parameters in the Heterogencous WSN

\begin{tabular}{cccccc}
\hline Network & $\alpha$ & $\beta$ & $\gamma$ & $\eta$ & $\delta$ \\
\hline UWB Cluster & 47,000 & 0.4886 & 16 & 13,000 & - \\
ZigBee Cluster & 10,000 & $-2 \times 10^{-15}$ & 89 & 15,950 & $3 \times 10^{-7}$ \\
WiMax Mesh & 100,000 & 0.0814 & 2 & 23,000 & - \\
\hline
\end{tabular}


lengths are short, as in our scenario. Table 5.1 summarizes our selection of $\alpha, \beta, \gamma$, $\delta$ and $\eta$ that have been calibrated for our network scenario.

For communication and packet transmission, we have implemented the network parameters in Table 5.2 in both the UWB and Zigbee clusters. In terms of operating parameters, the maximum number of hops $K^{\prime}=4$ for a single candidate path and the energy consumption coefficient $\zeta=0.5$ to split the energy required to transmit over a single hop in a path evenly between a transmitter and receiver as discussed in Section 2.4.1. $F_{1}=F_{2}=F_{c}=5$ as the number of sub-channels in the spectrum band with corresponding bandwidths of $w_{1}=500 \mathrm{MHz}, w_{2}=2.5 \mathrm{MHz}$ and $w_{m}=20$ $\mathrm{MHz}$ in our simulation for UWB, Zigbee and WiMax, respectively. As can be seen in Table 5.2, the UWB sensor cloud also transmits four times the information per transmission than the Zigbee sensor cloud to support higher bandwidth applications.

\section{Table 5.2: Communication Parameters for Three Sub-Networks}

\begin{tabular}{ccccccc}
\hline \hline Sub-Network & $\begin{array}{c}\text { Payload Length } \\
l \text { (bytes) }\end{array}$ & $\begin{array}{c}\text { Packet Length } \\
m \text { (bytes) }\end{array}$ & $\begin{array}{c}\text { Data to Transmit } \\
\text { (bytes) }\end{array}$ & $\begin{array}{c}\text { Packets to } \\
\text { Transmit, } n\end{array}$ & $\begin{array}{c}\text { Number of } \\
\text { Channels }\end{array}$ & $\begin{array}{c}\text { Channel } \\
\text { Bandwidth }\end{array}$ \\
\hline UWB & 32 & 38 & 128 & 4 & 5 & $500 \mathrm{MHz}$ \\
ZigBee & 32 & 38 & 32 & 1 & 5 & $25 \mathrm{MHz}$ \\
WiMax & 48 & 256 & 128 or 32 & 4 or 1 & 5 & $20 \mathrm{MHz}$ \\
\hline
\end{tabular}

\subsection{Initial Energy Capacity Modeling}

The NPP showed that initial energy capacities of sensor and mesh nodes depend heavily on the energy efficiency of the communication technology selected. Given the variations in $q_{t_{v_{x}}}$ for UWB, Zigbee and WiMax that we use to test our system, we must equip our sensor and mesh nodes with different energy capacities to support our 


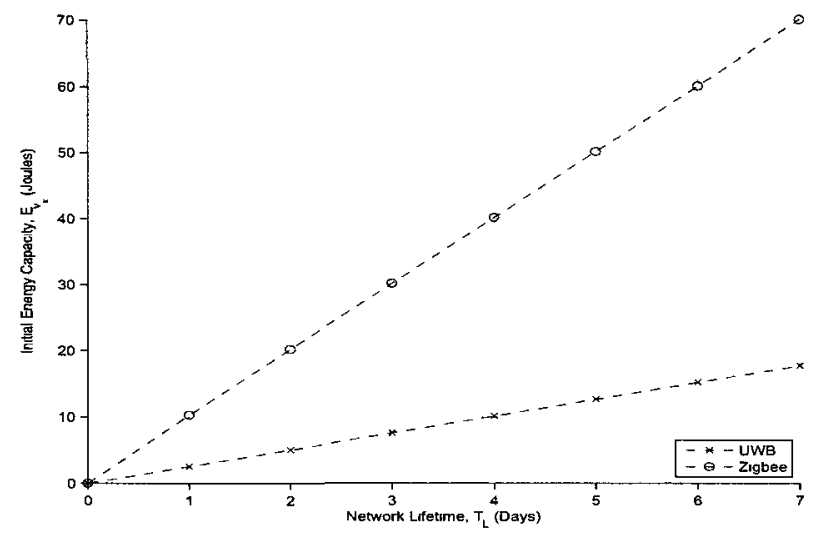

(a) With Coordination Protocols for $T_{H}=3$ seconds

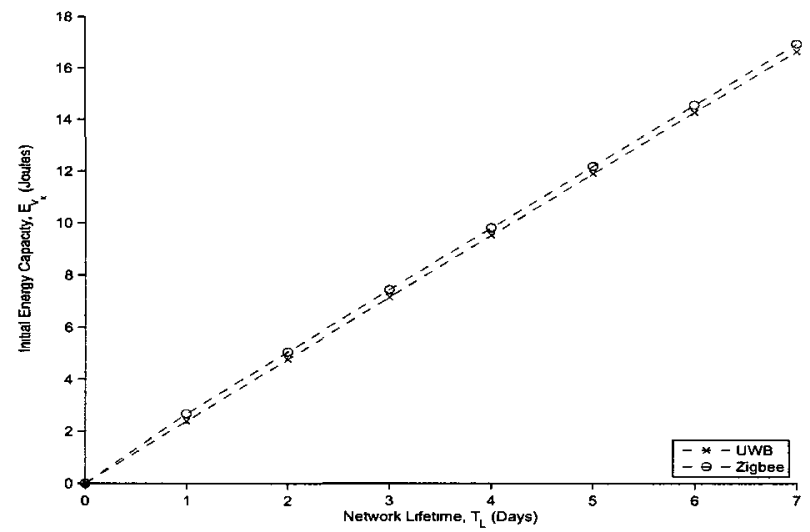

(b) Without Coordination Protocols for $T_{H}=\infty$ seconds

Figure 5.9: Energy Savings by UWB over One Week Network Lifetime

desired network lifetime of one week. For our simulation, we aim to meet expected performance levels of transmission rates specified in Table 1.1. We also limit each sensor to 1,000 requests over the course of the one week network lifetime in determining the initial energy capacities. Given that $V=20$ and $M=7$, the initial energy capacities required by UWB and Zigbee sensors over a desired one week network lifetime, both with and without coordination protocols, are presented in Figure 5.9. 
Table 5.3: Required and Applied Energy Capacities for Sensors, Cluster-Heads and Mesh Relay Nodes for One Week Network Lifetime

\begin{tabular}{ccc}
\hline \hline Node Type & $\begin{array}{c}\text { Required Energy } \\
\text { Capacity }\end{array}$ & $\begin{array}{c}\text { Apphed Energy } \\
\text { Capacity }\end{array}$ \\
\hline UWB sensor & $177 \mathrm{~J}$ & $175 \mathrm{~J}$ \\
Zigbee sensor & $699 \mathrm{~J}$ & $695 \mathrm{~J}$ \\
Cluster-head (UWB) & $7503 \mathrm{~J}$ & $1 \mathrm{~kJ}$ \\
Cluster-head (Zigbee) & $6848 \mathrm{~J}$ & $1 \mathrm{~kJ}$ \\
Mesh relay & $6465 \mathrm{~J}$ & $1 \mathrm{~kJ}$ \\
\hline
\end{tabular}

Without coordination protocols, we see that both UWB and Zigbee sensors can be equipped with roughly $17 J$ of energy. However, with the extra burden placed on the network by coordination protocols, UWB nodes require a modest increase, while Zigbee devices require over four times the energy capacity. Based on this analysis, we equip UWB nodes and Zigbee nodes with $17.5 J$ with $69.5 J$, respectively. $E_{m}$, the energy capacity of a mesh node, is chosen as $1 k J$. A summary of the required energy capacities and those implemented in the network for sensors, cluster-heads and mesh relay nodes given our system parameters are presented in Table 5.3. We implement sensor energies slightly under the required levels to analyze the performance of these nodes in the final stages of our network lifetime.

\subsection{Evaluative Metrics}

We evaluate our cross-layer policy according to a number of performance metrics. This will enable us to test the ability of our network to achieve both significant throughput performance and a predictable network lifetime. We shall also present the average waiting times of a priority policy and the blocking probability. 


\subsubsection{Throughput}

We shall examine the real-time total network throughput and mean per-hop throughput of our UWB and Zigbee clusters, and the WiMax mesh network, to test the ability of our policy to meet the desired throughput levels listed in Table 1.1 for three different communication protocols. By doing so, we test our system's ability to meet performance objectives for next-generation WSNs.

While the choice of technologies in this work is secondary to the policy itself, we shall also analyze the performance of UWB as a desirable WSN technology. We aim to show that UWB provides a significant performance improvement over Zigbee in terms of the total throughput, mean per-hop throughput and spectral efficiency.

In using this metric, we will also be able to analyze the mean number of hops per path in all three sub-networks to test our policy's ability to favor routing in the mesh network. This shall be calculated on an ongoing basis by dividing the sum of the total network throughput by the product of the number of active transmissions and the sum of the mean per-hop throughput over time.

\subsubsection{Percentage of Remaining Energy Capacities}

This metric evaluates the ability of our NPP to meet network lifetime requirements of next-generation WSNs. By monitoring the remaining energy capacities over the course of the simulation, we shall test the ability of our NPP to meet the desired network lifetime of one week for three communication protocols with different energy 
efficiencies. It will also enable us to evaluate the success of our NPP in helping us make critical network design decisions, while satisfying desired throughput levels and expected network lifetime. This is critical in application-centric WSNs to meet both resource and performance requirements of next-generation sensory and control applications.

\subsubsection{Mean Number of Network Connections}

The mean number of connections monitors the ability of our sensor and mesh nodes to maintain connectivity in the network. Network connections in the UWB and Zigbee clusters are Bernoulli distributed with success probability $p$ while WiMax nodes are fully connected in a mesh network with $M-1$ connections. This metric will also enable us to monitor the point at which our nodes begin losing connectivity.

\subsubsection{Blocking Probability}

The blocking probability metric examines the ability of our system to handle the network load and evaluates the number of dropped requests. We present a series of results for the blocking probability as a function of the traffic intensity and the number of operating channels, both for the overall network as well as individually for the UWB and Zigbee clusters in order to compare their performance.

Consider a request for a transmission to the master node for a source sensor being accepted in the UWB cluster (event $X_{1}$ ). We denote the probability that the request 
is accepted as $P\left(X_{1}(t) \mid E\left[K_{1}\right]\right)$ where $E\left[K_{1}\right]$ is the expected number of hops along a candidate path that originates from the UWB cluster. $P\left(X_{1}(t) \mid E\left[K_{1}\right]\right)$ is dependent on $E\left[K_{1}\right]$ as the success of a request is dependent on the ability of the cross-layer optimization algorithm to perform a resource allocation for each hop along the path. The corresponding probability in the Zigbee network is $P\left(X_{2}(t) \mid E\left[K_{2}\right]\right)$ where event $X_{2}$ considers the servicing of a request from a Zigbee sensor. In general, the expected number of transmissions approved in the network at time $t$ in seconds is,

$$
E\left[N_{A}(t)\right]=E\left[\lambda_{n e t}\right] * P(X(t) \mid E[K])
$$

as $\mathrm{E}\left[\lambda_{n e t}\right]$, the number of requests expected to arrive in the system, multiplied by the probability that a request is accepted in the network at time $t, P(X(t) \mid E[K])$. The expected percentage of accepted requests in the network, $E\left[P_{A}(t)\right]$, is derived as,

$$
\begin{aligned}
E\left[P_{A}(t)\right] & =\frac{\sum_{t^{\prime}=1}^{t} E\left[N_{A}\left(t^{\prime}\right)\right]}{t E\left[\lambda_{n e t}\right]} * 100 \% \\
& =\frac{\left.\sum_{t^{\prime}=1}^{t} E\left[\lambda_{\text {net }}\right] * P\left(X\left(t^{\prime}\right) \mid E[K]\right)\right]}{t E\left[\lambda_{n e t}\right]} * 100 \% \\
& \left.=\frac{1}{t} \sum_{t^{\prime}=1}^{t} P\left(X\left(t^{\prime}\right) \mid E[K]\right)\right] * 100 \%
\end{aligned}
$$

as the percentage of total requests that the system has received since the onset of the simulation that are approved.

We may now compute the expected blocking probability, $E\left[P_{B}(t)\right]$. In our network, a request is rejected if no candidate path can be resolved for the given source sensor 
at the time of the request. As these paths are multi-hop, a candidate path is not resolvable if any of the hops along the path cannot be optimized. This occurs if a transmitter along a path cannot resolve a channel because it: 1) cannot meet the minimum SNR of its prospective receiver due to high interference; 2) cannot reach the receiver's MIRS given the available link budget at the time of the request; 3 ) is unable to find an operating channel that is not in use by other sensors within its range; or, 4) has insufficient energy capacity to operate. If a candidate path cannot be resolved, we evaluate the next candidate path. If no candidate paths can be resolved, the request is rejected.

$E\left[P_{B}(t)\right]$ can be calculated as the total number of rejected requests divided by the total number of requests that have arrived to date as,

$$
\begin{aligned}
E\left[P_{B}(t)\right] & =\frac{\sum_{t^{\prime}=1}^{t}\left(E\left[\lambda_{n e t}\right]-E\left[N_{A}\left(t^{\prime}\right)\right]\right)}{t E\left[\lambda_{n e t}\right]} * 100 \% \\
& =\frac{t E\left[\lambda_{n e t}\right]-\sum_{t^{\prime}=1}^{t} E\left[N_{A}\left(t^{\prime}\right)\right]}{t E\left[\lambda_{n e t}\right]} * 100 \% \\
& =\frac{t-\sum_{t^{\prime}=1}^{t} P\left(X\left(t^{\prime}\right) \mid E[K]\right)}{t} * 100 \% \\
& =\left(1-E\left[P_{A}(t)\right]\right) * 100 \%
\end{aligned}
$$

\subsubsection{Expected Waiting Times with High Priority Clusters}

Priority schemes for WSNs provide preference over mesh network resources to critical transmissions from sensor clusters. Given that the purpose of the distributed sensor 
network is the execution of several maintenance and control applications simultaneously, a priority scheme allows our system to give precedence to high priority traffic; this enables critical data from sensor clusters to arrive immediately with timely and optimized responses. Two types of customers are considered in our system, low and high priority, and all requests of a single cluster are given the same priority level. Thus, at any time, a cluster-head is only servicing requests of a single priority level.

Thus, to study our priority-based model, we execute this scenario from the perspective of a central $\mathrm{M} / \mathrm{M} / 1$ server with an infinite queue that handles all incoming requests from all clusters for which the service rate for all requests is $\mu$. The $M / M / 1$ server does not exist in reality as cluster-heads are responsible for servicing their cluster requests. We use this server here to evaluate our priority scheme and compare the average waiting times of both high and low priority requests. The server has one queue to service requests on a first-come-first-serve basis to ensure that resources are allocated to one request appropriately prior to servicing the next request.

Consider our distributed WSN that contains UWB and Zigbee clusters. Requests arrive in sensor clusters at a rate of $\lambda_{c}=\lambda_{n e t_{1}}=\lambda_{\text {net }_{2}}$, where $\lambda_{\text {net }_{1}}$ and $\lambda_{\text {net }_{2}}$ are the cluster arrival rates of requests for the UWB and Zigbee clusters, respectively. The total arrival rate of requests is $\lambda_{n e t}=\lambda_{n e t_{1}}+\lambda_{n e t_{2}}$.

The average waiting time metric enables us to compare the time to service high priority and low priority requests. We will compare the servicing of these two types of customers with the average waiting time when no high priority traffic is present, as well as the average waiting time for all requests over the duration of the simulation. 
We shall study four types of requests in measuring the average waiting times: high and low priority requests from the UWB cluster for which the probability of a high priority event is $p_{h_{1}}$, and high and low priority requests from the Zigbee cluster for which the probability of a high priority event is $p_{h_{2}}$.

The average waiting time with no high priority requests in the system is,

$$
W(\mu, \rho)=\frac{\rho}{\mu(1-\rho)}
$$

where,

$$
\rho=\frac{\lambda_{n e t}}{\mu}
$$

For high priority UWB requests for which $\rho_{1}=\lambda_{\text {net }} / \mu$, the average waiting time is,

$$
W_{h a g h_{1}}=\left(1-p_{h_{2}}\right) W\left(\mu, \rho_{1}\right)+p_{h_{2}} W(\mu, \rho)
$$

as the average waiting time when the UWB cluster is the only high priority cluster and both the UWB and Zigbee clusters are high priority.

For high priority Zigbee requests and $\rho_{2}=\lambda_{\text {net }_{2}} / \mu$, the average waiting time is calculated as,

$$
W_{h_{\imath g} h_{2}}=\left(1-p_{h_{1}}\right) W\left(\mu, \rho_{2}\right)+p_{h_{1}} W(\mu, \rho)
$$

In general, for $p_{h}=p_{h_{1}}=p_{h_{2}}$ and $\lambda_{n e t_{1}}=\lambda_{n e t_{2}}$, the average waiting time for high 
priority requests reduces to,

$$
W_{h \imath g h}\left(\mu, \rho, p_{h}\right)=\left(1-p_{h}\right) W(\mu, \rho / 2)+p_{h} W(\mu, \rho)
$$

Low priority requests will be preempted by high priority requests in the queue following a preemptive resume strategy; high priority requests will preempt low priority requests, which after servicing all high priority requests, the low priority requests will resume. The average waiting time for the preemption resume scheme is,

$$
W_{l o w}\left(\mu_{1}, \mu_{2}, \rho_{1}, \rho_{2}\right)=\frac{\mu_{2} \rho_{1}+\mu_{1} \rho_{2}}{\mu_{1} \mu_{2}\left(1-\rho_{1}\right)\left(1-\rho_{1}-\rho_{2}\right)}
$$

which, for $\mu=\mu_{1}=\mu_{2}$ and $\rho=\rho_{1}=\rho_{2}$, reduces to,

$$
W_{l o w}(\mu, \rho)=\frac{2 \rho}{\mu(1-\rho)(1-2 \rho)}
$$

The overall average waiting time in the system considering the above scenarios is,

$$
W_{a v g}\left(\mu, \rho, p_{h}\right)=p_{h} W_{h a g h}\left(\mu, \rho, p_{h}\right)+\left(1-p_{h}\right)\left(p_{h}\right) W_{l o w}(\mu, \rho)+\left(1-p_{h}\right)\left(1-p_{h}\right) W(\mu, \rho)
$$

Typically, priority-based systems are also used in conjunction with flow control mechanisms to manage the amount of traffic in the network; if the input traffic exceeds the total network capacity, lower priority traffic is blocked to ensure that the network capacity is not exceeded. In our distributed WSN, however, the cluster-head is responsible for performing request optimization in a centralized fashion on behalf of 
sensors in the network as discussed in Chapter 3. This optimization is performed from the perspective of a single channel where the cluster-head assigns separate channels to each hop. Thus, while in our study we do not consider the total network capacity, the cluster-head considers the single channel's capacity $C_{v_{y}, f}(t)$ for each transmission request, as shown in the link utility function in (3.2). The cluster-head is aware of all power allocations by its sensors on each channel and ensures that the single channel's capacity is not exceeded. This is enforced due to the presence of $P_{c a p}$ in the objective function constraints covered in Section 3.7.2. Since the per channel capacity is not exceeded, the total network capacity is also not violated. However, if flow control mechanisms are desirable, this can be achieved by using the priority-based system derived here to assign priorities to identify low priority traffic that can be dropped if total network capacity is being exceeded.

\subsection{Summary}

This chapter discussed system design issues including our NPP, state information and simulation methodology. We also discussed our queueing theory model and the derivation of our optimization parameters used in the study. As we mentioned, the queueing model depends on the message capacities that are derived from our NPP, which illustrates the relationship between application performance, network lifetime and energy capacity, as well as the message load that the network can support. Based on this network load, we calculate our arrival rate of requests, $\lambda_{1}$ and $\lambda_{2}$, for our two 
clusters accordingly. The NPP presented in this chapter is the third contribution of this thesis and enables network planners to make critical network design decisions by studying their impact on throughput performance and network lifetime objectives.

The next chapter will present a performance evaluation of our cross-layer optimization algorithm and NPP for a number of evaluative metrics presented in this chapter. We will seek to meet the performance and network lifetime objectives set out in Section 5.8 . 


\section{Chapter 6}

\section{Performance Evaluation}

\subsection{Introduction}

Our cross-layer algorithm for throughput and energy optimization in WSNs is simulated using the MATLAB computing platform. MATLAB is a language that enables intensive computation and is ideal for our requirements as the Nelder-Mead method for nonlinear optimization is built into MATLAB's Optimization Toolbox. While MATLAB is not itself a network simulator, its optimization functions are extremely useful, while the MATLAB language makes the implementation of network topologies, interference modeling and signal propagation characteristics quite feasible.

In this chapter, we will be applying the performance metrics discussed in our previous section to our cross-layer optimization policy. In this evaluation, we shall be evaluating the ability of our algorithm to meet both the performance and network lifetime requirements that were outlined Chapter 5 . We will commence with a discussion of the convergence of our algorithm. 


\subsection{Convergence and Nelder-Mead Method for Nonlinear Optimization}

To optimize our objective function $U_{v_{2}, v_{j}}^{a^{*}}$, our algorithm uses the Nelder-Mead method for nonlinear optimization. Nelder-Mead uses a simplex method that utilizes the concept of a polytope with $n+1$ vertices in an $n$ dimensional space [107]. Since our optimization of a single link utility $L_{v_{x}, v_{y}, f}^{*}$ at the PHY layer is optimized for one communication resource of interest $r_{f}=\phi\left(P_{v_{x}, f}^{t}\right)$ only, the Nelder-Mead method uses a line segment (with two vertices) to generate a set of trial results. The Nelder-Mead algorithm extrapolates the objective function at these test points to analyze points along their gradients, and replaces these test points with more optimal solutions as the algorithm progresses, until a local minimum is found.

Four scalar parameters form a complete Nelder-Mead method: coefficients of reflection $(\rho)$, expansion $(\chi)$, contraction, $(\gamma)$ and shrinkage $(\sigma)$ [107]. To satisfy the algorithm, these parameters must adhere to the following rules,

$$
\rho>0, \chi>1, \chi>\rho, 0<\gamma<1, \text { and } 0<\sigma<1
$$

where the general selections for these parameters are [107],

$$
\rho=1, \chi=2, \gamma=\frac{1}{2}, \text { and } \sigma=\frac{1}{2}
$$

Lagarias et al. prove that the Nelder-Mead method converges to a minimizer for strictly convex functions of one variable [108]. Given that a strictly convex function has only a single minimum, any local minimum is also a unique global minimum [109]. 
Thus, to prove the convergence of our optimization algorithm and, given that we have a maximization problem, we must show that our link utility function $L_{v_{x}, v_{y}, f}^{*}$ is strictly concave. A proof of concavity, given through the convexity of $-L_{v_{x}, v_{y}, f}^{*}$, is provided in Appendix $\mathrm{C}$, in which we provide the necessary conditions to ensure a global optimum can be obtained, as well as prove that $-L_{v_{x}, v_{y}, f}^{*^{\prime \prime}}\left(P_{v_{x}, f}^{t}, t\right)>0$ for our operating parameters in Tables 5.1 and 5.2 .

Given that a sum of convex functions is also convex, our objective function $-U_{v_{\imath}, v_{\jmath}}^{a^{*}}$, as the summation of $-L_{v_{x}, v_{y}, f}^{*}$ functions, is also convex. Thus, our algorithm converges to the unique global maximum for $U_{v_{z}, v_{j}}^{a^{*}}$ given our objective function constraints.

For frequently optimized functions, the Nelder-Mead method enables the specification of an initial value from which to begin the search if there has been relatively few changes to the state of the network since the last optimization [107]. Thus, we store and maintain our previous solution, previous_solution $\left(v_{x}, v_{y}, f\right)$, and use it as our initial value for the hop $v_{x}-v_{y}$ on channel $f$ for the next iteration.

\subsection{Simulation Results}

Our simulation evaluates the performance of our cross-layer optimization policy for joint throughput and energy optimization, and the mesh network as an enabling technology for building distributed and application-centric WSNs. As discussed in Chapter 5, we use two clusters based on UWB and Zigbee, and an overlay WiMax 
mesh network, to test our cross-layer optimization policy. We also conduct a performance comparison between UWB and Zigbee to study the suitability of UWB for deploying next-generation WSNs. In Chapter 5, we stated that our goal is to design our WSN to survive for a one week network lifetime without loss of generality, while we also test the ability of our cross-layer optimization policy to meet the throughput performance outlined in Table 1.1. For Zigbee, we utilize the $2.4 \mathrm{GHz}$ band that is capable of reaching data rates of $250 \mathrm{kbps}$. Here, we present results for the evaluative metrics discussed in Chapter 5 .

\subsubsection{Total Network Throughput}

In our problem formulation, we discussed the requirements of next-generation WSNs that will be expected to perform at peak performance for state-of-the-art sensory and resource management applications. In this thesis, we have explored performance issues for WSNs in the form of achievable throughput. We have studied achievable throughput by considering both the benefits and costs of utilizing a level of throughput as a function of the consumption of physical resources in our cross-layer design. Rather than only considering minimum QoS requirements, we aim to tie performance

Table 6.1: Throughput Statistics

\begin{tabular}{cccccc}
\hline Network & $\begin{array}{c}\text { Maximum Total } \\
\text { Throughput }\end{array}$ & $\begin{array}{c}\text { Minimum Total } \\
\text { Throughput }\end{array}$ & $\begin{array}{c}\text { Mean Total } \\
\text { Throughput }\end{array}$ & $\begin{array}{c}\text { Standard } \\
\text { Deviation }\end{array}$ & Variance \\
\hline UWB Cluster & $3.4 \mathrm{Gbps}$ & $84.4 \mathrm{Mbps}$ & $1.8 \mathrm{Gbps}$ & $112.2 \mathrm{Mbps}$ & $1.3 \times 10^{16}$ \\
ZigBee Cluster & $794.9 \mathrm{kbps}$ & $240.1 \mathrm{kbps}$ & $770 \mathrm{kbps}$ & $2.2 \mathrm{kbps}$ & $5.0 \times 10^{6}$ \\
WiMax Mesh & $485.7 \mathrm{Mbps}$ & $39.6 \mathrm{Mbps}$ & $331.5 \mathrm{Mbps}$ & $10 \mathrm{Mbps}$ & $1.6 \times 10^{13}$ \\
\hline
\end{tabular}


and energy capacity to physical resource consumption. This enables us to gain a clear picture of both the benefits and costs of various resource assignments to make more informed decisions regarding resource allocation. The first part of the evaluation of our cross-layer design is an analysis of the total network throughput in our network. Throughput statistics of our distributed WSN are presented in Table 6.1 for sensory data traffic and does not include the transmission rate of coordination messages.

Table 6.1 illustrates the most significant performance improvement of the UWB cluster over the Zigbee cluster. The UWB sensor cloud achieves total network throughputs that vary between $84.4 \mathrm{Mbps}$ and 3.4 Gbps for our network load, while the Zigbee sensor network achieves a maximum real-time throughput of only 794.9 kbps. Recall that Zigbee transmissions are only capable of achieving single throughputs of $250 \mathrm{kbps}$. The mesh network provides long-haul transmission to the processing center from the cluster-heads of the sensor clouds and, in our simulation, the mesh network achieves network throughputs of between 39.6 and $485.7 \mathrm{Mbps}$.

This result is significant and shows the success of our cross-layer optimization algorithm in using UWB to achieve significant throughput gains. This also illustrates that UWB is indeed suited to next-generation WSNs that require high throughput performance for next-generation sensory applications. UWB expands the number of applications for WSNs that can be used for state-of-the-art resource management, which solves a major long-term concern for WSNs considering the low bandwidth limitations of Zigbee technology. 


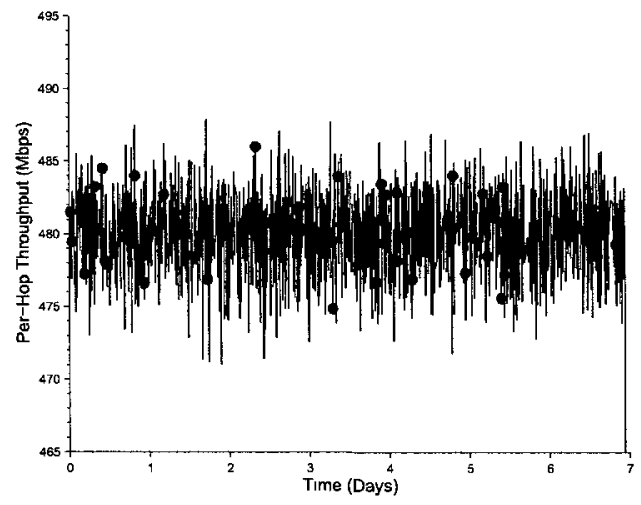

(a) UWB Sensor Cluster

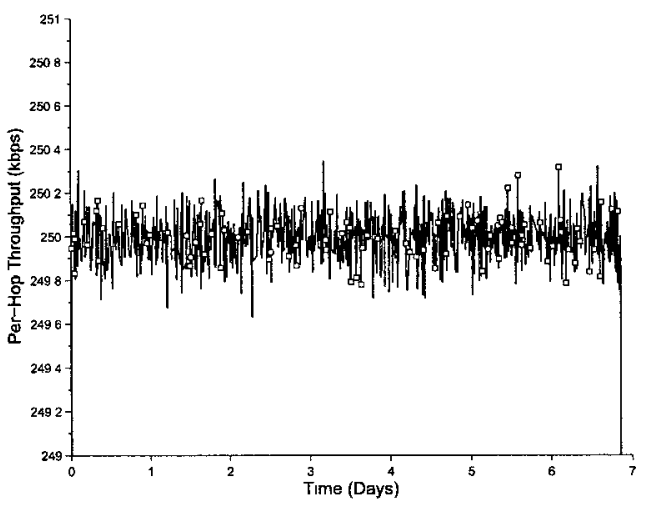

(b) Zigbee Sensor Chuster

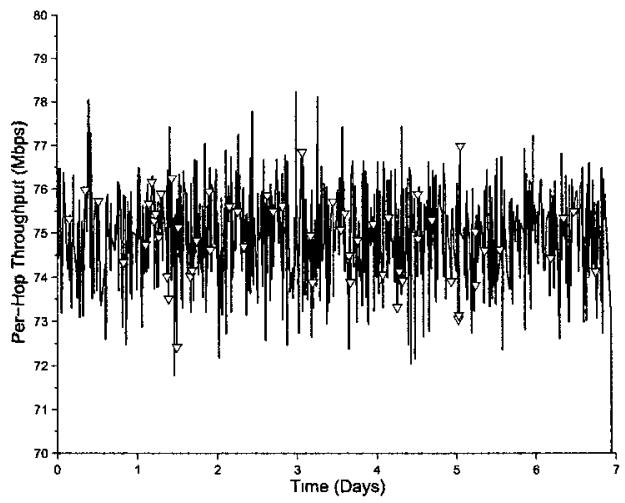

(c) WiMax Mesh Network

Figure 6.1: Mean Per-Hop Throughput over One Week Network Lifetime

\subsubsection{Mean Per-Hop Throughput}

Throughput performance can also be viewed in terms of the mean throughput as shown in Figure 6.1. This result highlights the performance improvement per hop along a path. Figure 6.1 shows that the mean per-hop throughput at any time over the course of the one week network lifetime for UWB, Zigbee and WiMax nodes is approximately $480 \mathrm{Mbps}, 75 \mathrm{Mbps}$ and $250 \mathrm{kbps}$, respectively, in accordance with Table 1.1. The fluctuations around the mean in terms of the standard deviation and 


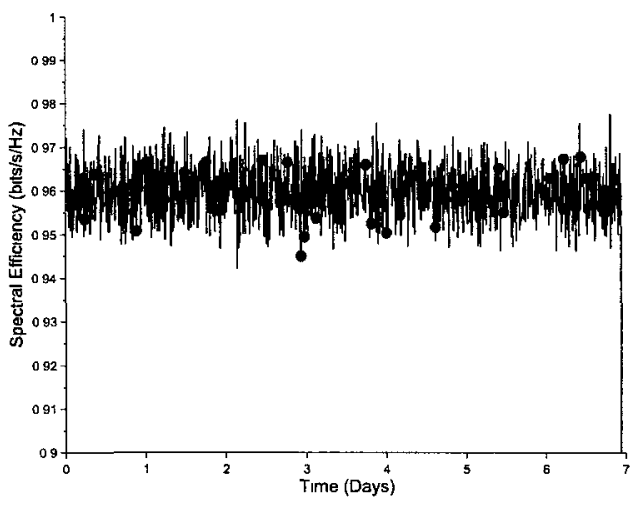

(a) UWB Sensor Cluster

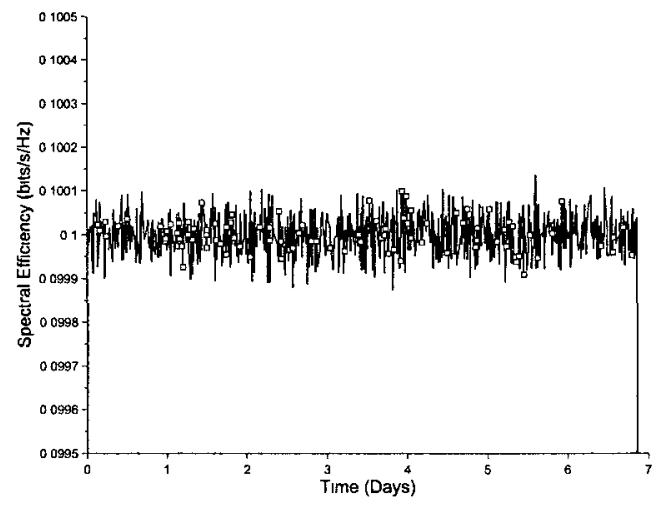

(b) Zigbee Sensor Cluster

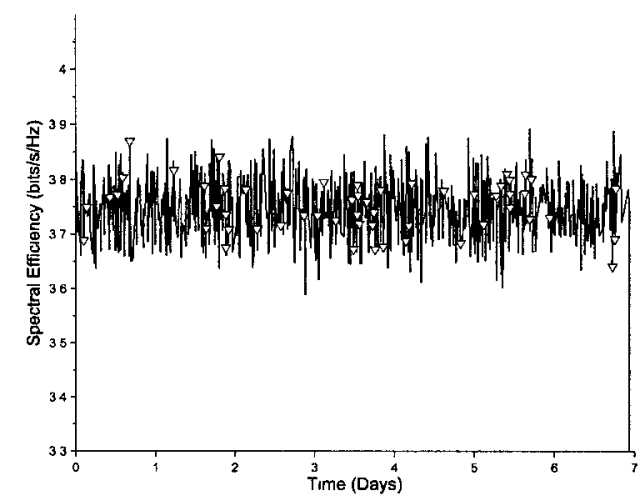

(c) WiMax Mesh Network

Figure 6.2: Spectral Efficiency (bits/s/Hz) over One Week Network Lifetime

variance are summarized in Table 6.1. The mean throughput also confirms that UWB is suited to large bandwidth applications for WSNs.

\subsubsection{Spectral Efficiency}

It is also important to evaluate the spectral efficiency of our three communication technologies to provide an accurate comparison in terms of the attainable transmission rate per Hz. Figure 6.2 presents the spectral efficiency of our three communication 
protocols in bits $/ \mathrm{s} / \mathrm{Hz}$. Between UWB and Zigbee, UWB provides a significantly higher spectral efficiency. Zigbee provides a spectral efficiency of $250 \mathrm{kbps} / 2.5 \mathrm{MHz}$ $=0.1 \mathrm{bits} / \mathrm{s} / \mathrm{Hz}$, while UWB attains a spectral efficiency of roughly $480 \mathrm{Mbps} / 500$ $\mathrm{MHz}=0.96 \mathrm{bits} / \mathrm{s} / \mathrm{Hz}$. This is accurate as UWB provides throughput between 100 Mbps and $500 \mathrm{Mbps}$ over a $500 \mathrm{MHz}$ channel [110]. WiMax provides improved spectral efficiency of approximately $75 \mathrm{Mbps} / 20 \mathrm{MHz}=3.75 \mathrm{bits} / \mathrm{s} / \mathrm{Hz}$.

\subsubsection{Average Number of Hops per Path}

We mentioned in Chapter 3 that an important design decision was ensuring that the algorithm favored routing in the mesh network over the sensor cluster. This enables us to shift the routing responsibility to the mesh network and conserve sensor energy by reducing the number of sensor nodes used in a path. This is achieved by scaling our achievable $L_{v_{x}, v_{y}, f}^{*}$ values such that link utilities in the mesh network are larger than in the sensor networks. The effect of this decision is that candidate paths in the path selection process with a larger number of mesh hops have a higher average path utility $U_{v_{z}, v_{j}}^{a^{*}}$. Thus, they are more likely to be selected in the optimization process.

We test the ability of our cross-layer optimization policy to favor mesh network routing by calculating the real-time average number of hops in each of the three subnetworks. The number of hops is found as the total network throughput divided by the product of the number of active transmissions and the mean per-hop throughput since the onset of the simulation. This is shown in Figure 6.3 which shows that our 


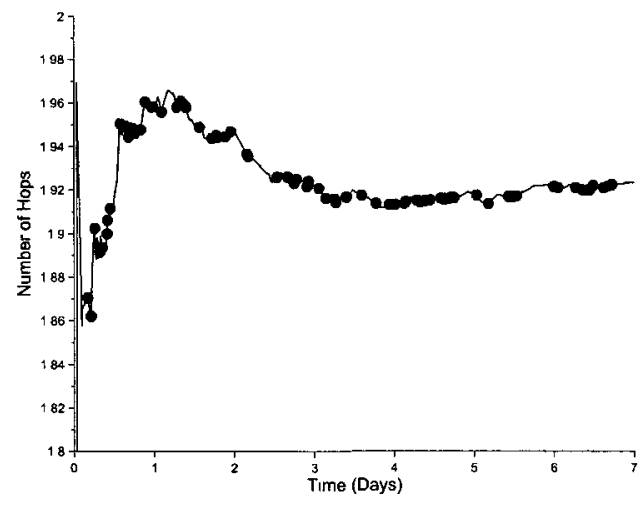

(a) UWB Sensor Cluster

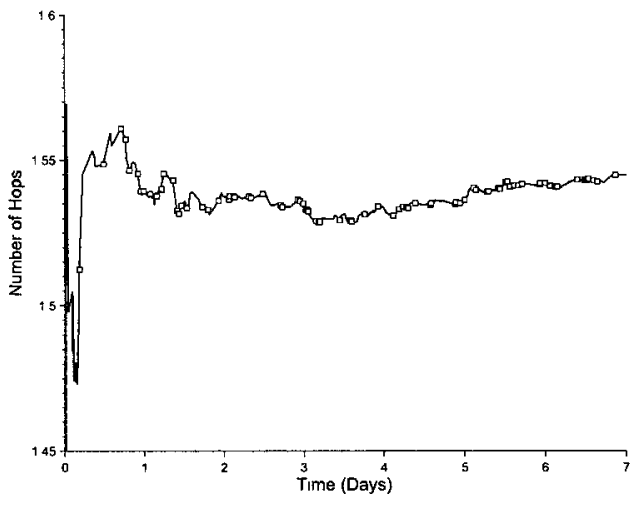

(b) Zigbee Sensor Cluster

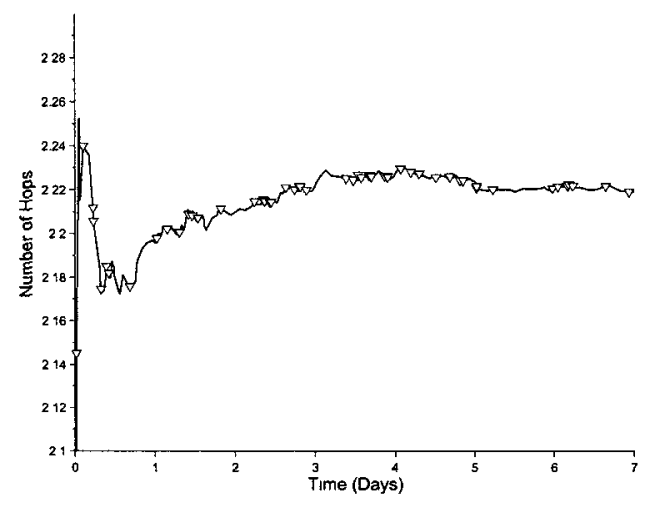

(c) WiMax Mesh Network

Figure 6.3: Average Number of Hops Per Path in Each Sub-Network

policy indeed favors routing in the mesh network. For $K^{\prime}=4$, on average $K_{m}=2.2$ hops occur in the mesh network. This is a $14.3 \%$ and $44.2 \%$ increase over the average number of hops per path in the UWB and Zigbee clusters, respectively, which provides substantial savings in sensor energies over the course of the network lifetime. Ideally, we would like the source sensor to transmit to the cluster-head in one hop but this is not always possible given the sensor distribution in the field and the maximum transmission range of the communication protocol. 
Another interesting observation to be made is that UWB paths have a higher number of hops on average than Zigbee paths. This requires more UWB nodes to be involved in an UWB transmission but occurs due to the shorter range of UWB devices and the increased dependence of UWB technology on multi-hop routing.

\subsubsection{Percentage of Remaining Energy Capacities}

While we observe the throughput benefits gained by using our cross-layer optimization policy, we must also analyze the energy cost at which these benefits are achieved. This would enable us to evaluate whether or not our algorithm is successful in jointly meeting significant throughput and network lifetime requirements.

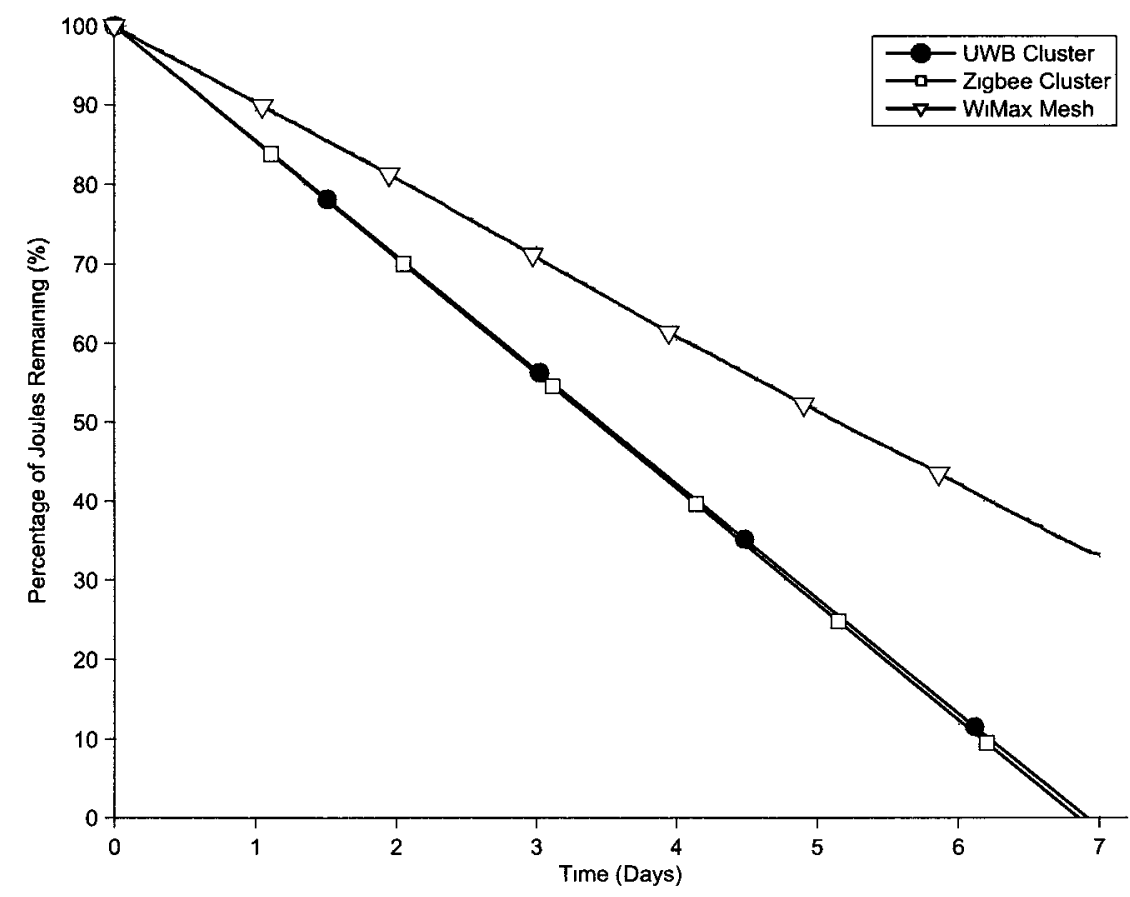

Figure 6.4: Comparison of Remaining Battery Energies Over One Week Network Lifetime 
Figure 6.4 presents the mean remaining energy capacities in the UWB cluster, Zigbee cluster and the WiMax mesh network. Our UWB and Zigbee clusters are able to survive for the desired one week network lifetime, with the outage occurring just before the end of the simulation. At the end of the simulation, the WiMax mesh network still has roughly $34 \%$ of its mean battery energy remaming. This is due to mesh nodes being equipped with more resources which enables the overlay network to assume greater responsibilities in the network. These results meet our network lifetime expectations based on the initial energy capacities determined via NPP in Table 5.3. For example, based on the expected and applied energy capacities in Table 5.3, we would expect that both the UWB and Zigbee clusters would lose connectivity

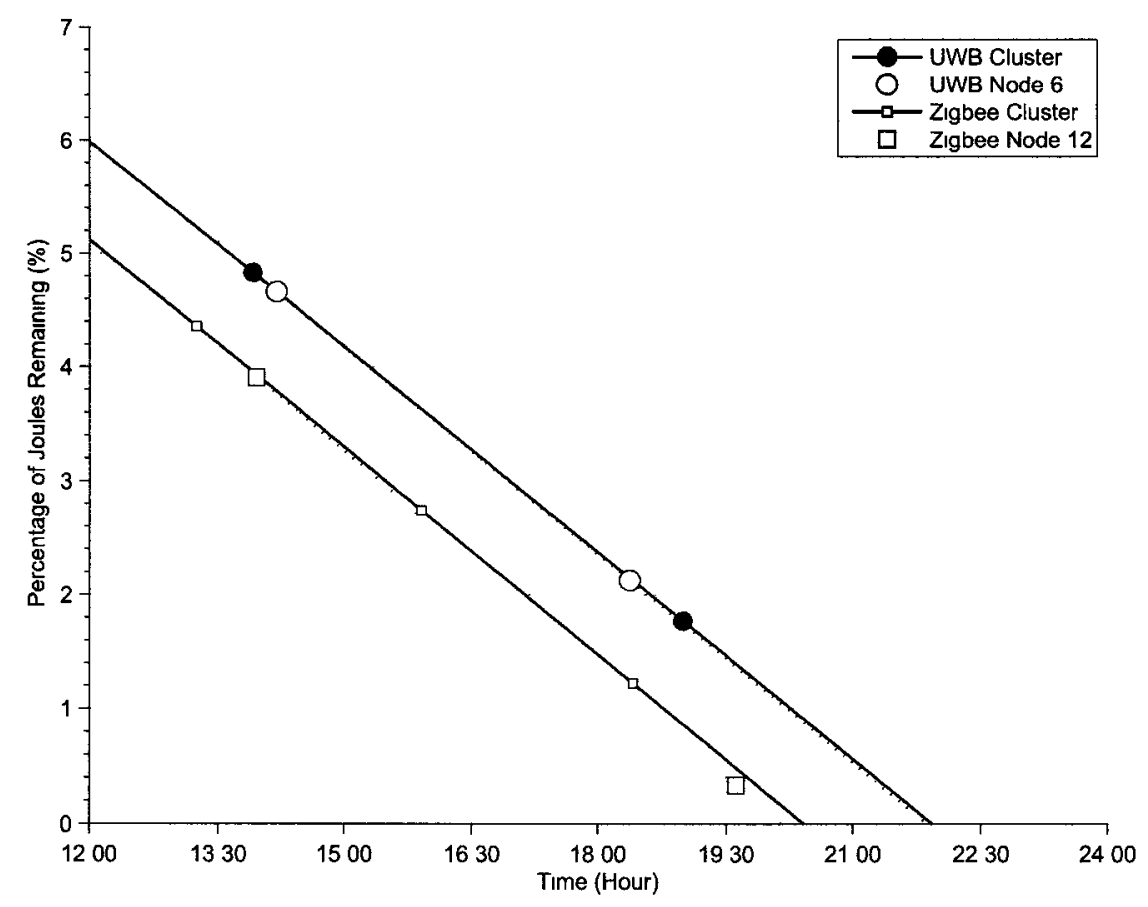

Figure 6.5: Remaining Network Energies in Last 12 Hours with First Node Outages 
in the last few hours of the network lifetime. We would also expect the mesh network to maintain roughly one-third of its energy capacity at the end of the simulation, which has been confirmed. This result is significant as it shows that we can indeed design WSNs to plan for predictable network lifetimes, while achieving significant throughput performance. Our NPP enables us to achieve both objectives.

Figure 6.5 illustrates the remaining energy capacities in the final twelve hours of the simulation and the first nodes in each cluster to fully lose connectivity. Based on the initial energy capacities selected, the UWB cluster gave us almost two extra hours of connectivity over the Zigbee network. In terms of the first node outages, node 12 from the Zigbee cluster was the first node to lose connectivity; its remaining battery energy was just under that of the mean from the Zigbee cluster at 20:15. For the UWB cluster, node 6 experienced the first node outage and followed the mean battery energy of the UWB cluster quite strictly at 22:00. These results also confirm what we would expect from our initial and applied energy capacities from Table 5.3.

\subsubsection{Mean Number of Network Connections}

In studying the mean number of connections for the network in Figure 6.6 during the final six hours of the simulation, we find a threshold energy capacity at which nodes being to lose connectivity. For the Zigbee and UWB clusters, the first link outages occurred at 20:15 and 22:00 on the final day of the simulation, respectively. This is confirmed by observing a sudden drop in network connections. 


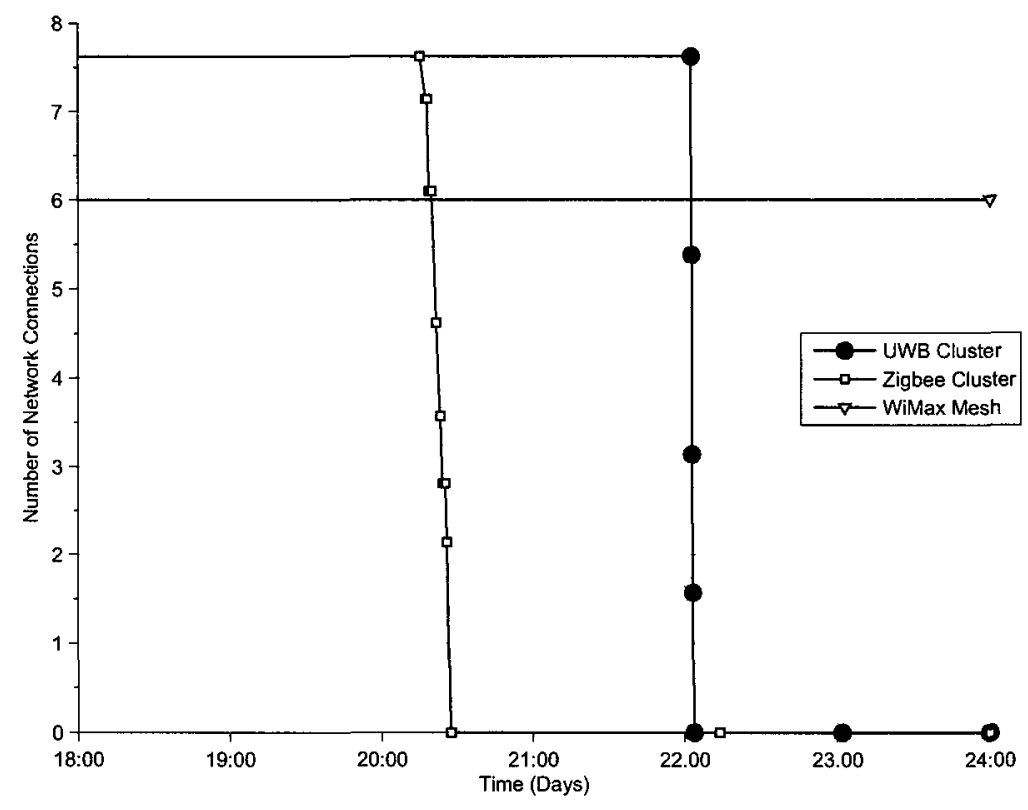

Figure 6.6: Mean Network Connections In Last Six Hours

The point at which network connections are lost occurs when only roughly $0.2 \%$ of the energy capacity remains for both the UWB and Zigbee clusters. This is due to nodes having insufficient power at this stage of the network's lifecycle to maintain connectivity. Prior to this stage, nodes are able to maintain connections. After both the UWB and Zigbee sensor nodes lose connectivity, Figure 6.6 also confirms that the WiMax mesh network remains fully-connected.

\subsubsection{Blocking Probability}

Figure 6.7 depicts the relationship between the overall network blocking probability for the UWB and Zigbee clusters and the traffic intensity, $\rho$. Recall that a request is blocked if the system is unable to resolve a path for a request, either due to a lack 


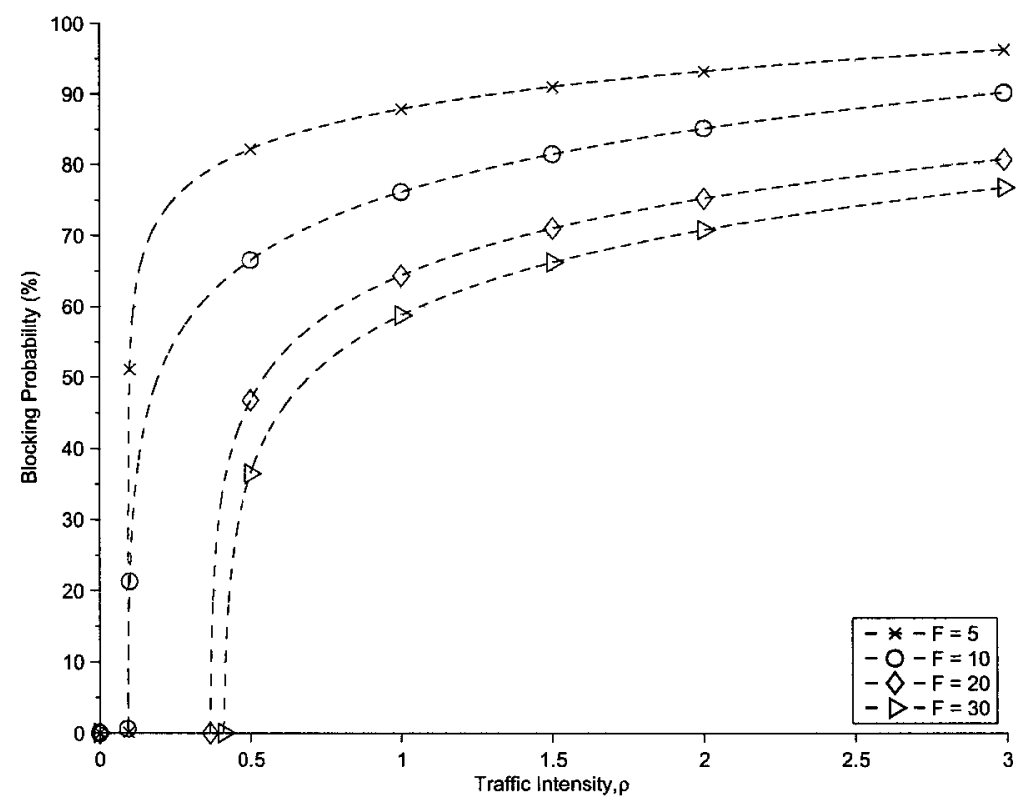

Figure 6.7: Network Blocking Probability vs. Traffic Intensity, $\rho$

of available operating channels, an inability of candidate transmitters to reach the minimum SNR at their prospective receivers, or a failure to find a path to satisfy the link budget at the time of the request.

As shown in Figure 6.7, as the traffic intensity increases, the probability that a request is blocked in the network increases logarithmically. This trend occurs because, as the traffic intensity increases, fewer channels are available as they are used up at an exponential rate, causing subsequent requests to be rejected. The number of channels, $F$, represents the number of channels simulated in each of the three sub-networks, that is $F_{1}=F_{2}=F_{c}=F$. As $F$ increases, the blocking probability decreases for a given traffic intensity. This indicates that the number of operating channels is the defining parameter in determining the operating point of the network for the blocking 
probability. This occurs because the mesh network handles requests from both the UWB and Zigbee clusters and, if all $F_{c}$ channels in the WiMax spectrum band are used for active transmissions, the WiMax network cannot support any more transmissions. Thus, the request is rejected even if channels are available in the sensor clusters. It also appears from Figure 6.7 that increasing the number of operating channels at low traffic intensities has a much larger effect on decreasing the blocking probability. As the traffic intensity increases, however, the effect of increasing the number of operating channels on the blocking probability decreases.

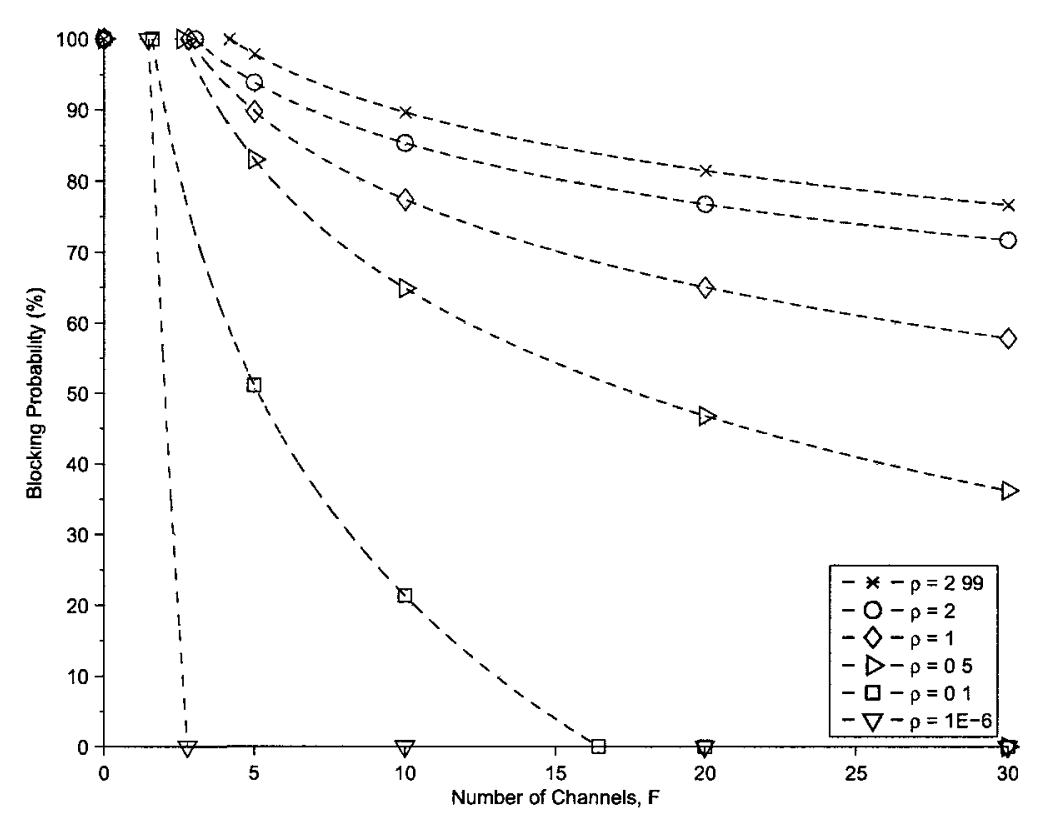

Figure 6.8: Network Blocking Probability vs. Number of Operating Channels, $F$

Figure 6.8 goes more in depth to portray the critical relationship between the network blocking probability and the number of operating channels. This shows that, as the number of operating channels increases, the blocking probability decreases 
according to a logarithmic relationship. However, as the traffic intensity and the number of channels increases, the blocking probability decreases at a slower rate, which confirms the conclusion reached from Figure 6.7. Figure 6.8 also illustrates that the blocking probability decreases as the traffic intensity decreases, which is expected. The sharpness of the drop for a traffic intensity $\rho=1 \mathrm{E}-6$ can be attributed to the near-zero blocking probability at extremely low traffic intensities.

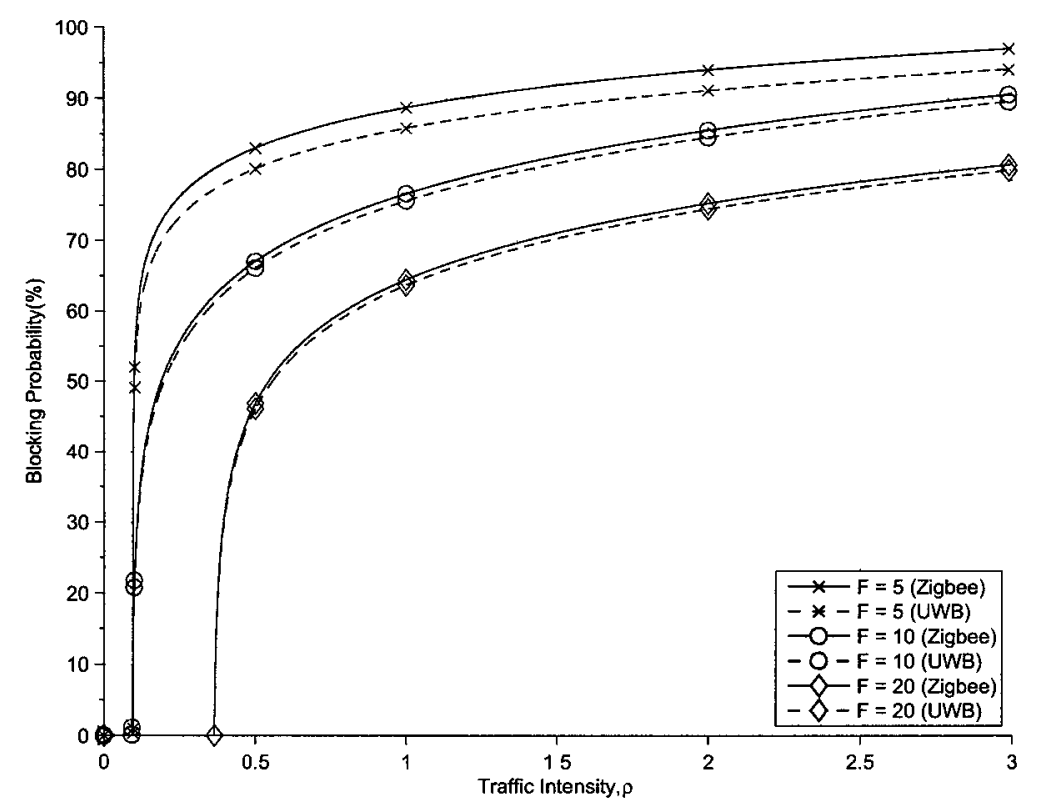

Figure 6.9: Blocking Probability of UWB and Zigbee Clusters vs. Traffic Intensity, $\rho$

Figure 6.9 illustrates the relationship between the blocking probability and traffic intensity for UWB and Zigbee separately for $F=5, F=10$ and $F=20$. Given the same traffic intensity and number of operating channels, the UWB cluster has a blocking probability that is approximately $2 \%$ lower than Zigbee on average for $F=5$. For $F=10$ and $F=20$, UWB also has a lower blocking probability 
than Zigbee but the improvement decreases as the number of channels is increased. This improvement using UWB re-iterates what we have observed previously, that UWB technology is better equipped to handle critical WSN transmissions in nextgeneration WSNs. Even if the improvement is only a couple of percentage points in terms of blocking probability, UWB provides additional throughput and energy efficiency performance over the current standard of WSNs based on Zigbee.

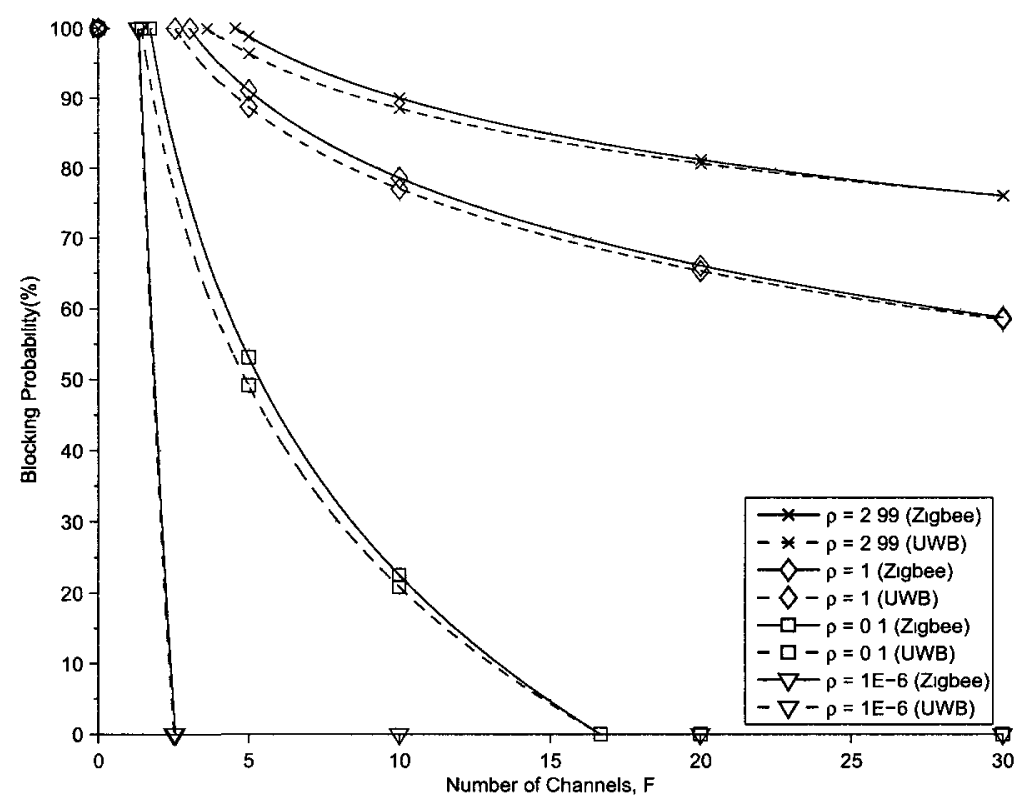

Figure 6.10: Blocking Probability of UWB and Zigbee Clusters vs. Number of Operating Channels, $F$

We also separate the performance of UWB and Zigbee in observing the relationship between the blocking probability and the number of channels in Figure 6.10. Similarly to Figure 6.9, it shows that, for a given traffic intensity, the UWB cluster is able to support more transmissions for the same number of operating channels by rejecting fewer requests when the number of channels is low. It is also interesting to note that 


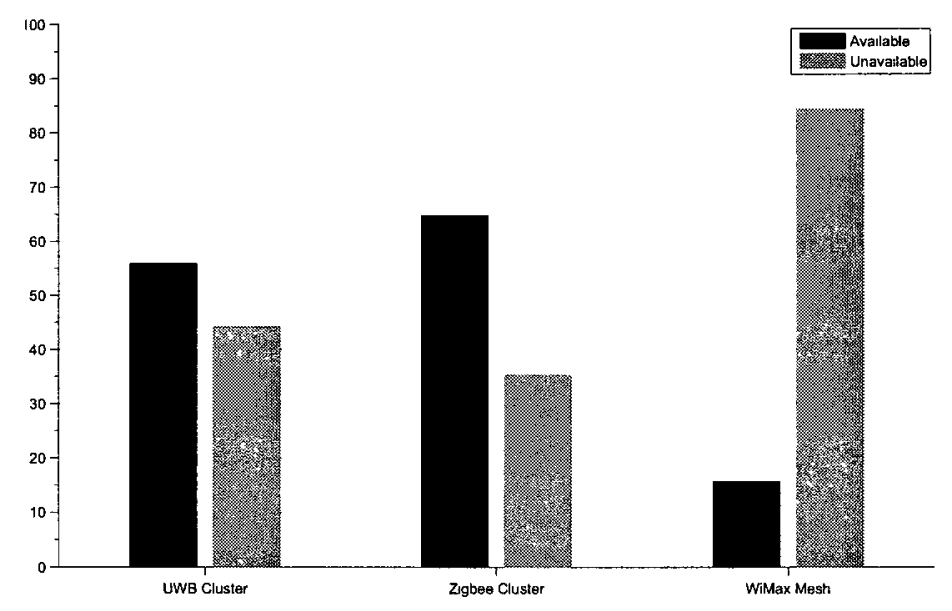

Figure 6.11: Percentage of Available Channels for $F=10$ and $\rho=1$ In Three Subnetworks

there comes a point where the difference between the blocking probabilities for UWB and Zigbee is marginal as we increase the number of operating channels.

With this metric, we have discussed the dependency of the blocking probability on the number of available channels in the mesh network. We study this trend specifically in Figure 6.11 where we have presented the number of available and unavailable channels on average during a simulation for $\rho=1$ and $F=10$. On average, the mesh network has $16 \%$ and $84 \%$ of its bins available and unavailable at any time, respectively. As we can see, the $84 \%$ of channels being unavailable in the mesh network is quite close to the $77 \%$ blocking probability for the $\rho=1$ and $F=10$ case from Figure 6.7. The $7 \%$ discrepancy can be attributed to the need for there to only be one available channel for a one-hop connection between the cluster-head and processing center for acceptance of the request. With $84 \%$ of channels being 
unavailable for $F=10$, this leaves approximately 1.6 channels available on average, which is sufficient for carrying traffic to the master node in one-hop.

Thus, the number of available channels in the mesh network is indeed the critical factor in determining the successful operating point of the network in terms of blocking probability. As a result, careful attention should be given to the mesh network to ensure that it is equipped with sufficient resources to handle the network load.

\subsubsection{Expected Waiting Times with High Priority Clusters}

In distributed WSNs that shall execute several maintenance and control applications simultaneously, priority schemes are crucial. Priority schemes enable critical data from one or more sensor clusters that require immediate attention to be attained.

In Figure 6.12, we present the average waiting time in a priority-based simulation to optimize a system with two priority levels. In this priority scheme, transmission requests from clusters given high priority preempt both active transmissions and current request optimizations from other clusters. This has two effects: enable high priority requests to be optimized based on the full view of resources that are generally

shared in the mesh network, and move high priority requests to the front of the queue for prioritized cross-layer optimization; recall that priority is assigned on a clusterbased level and, thus, high priority requests do not pre-empt active transmissions that originate from the same cluster. Upon handling high priority requests, the system resumes low-priority requests based on the new view of available resources. 


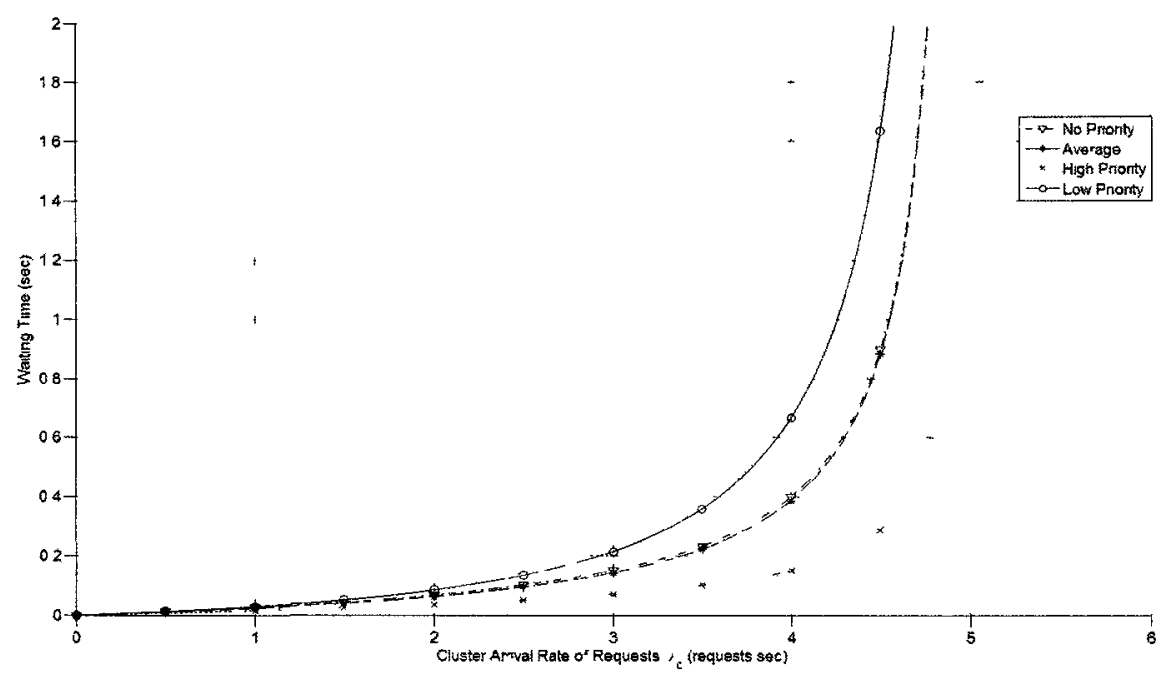

Figure 6.12: Expected Waiting Time for High and Low Priority Requests with $p_{h}=$ $25 \%$ and $\mu=10$ requests/sec

We study four types of requests: high and low priority requests from the UWB cluster, and high and low priority requests from the Zigbee cluster. The probability of a high priority event $p_{h}=p_{h_{1}}=p_{h_{2}}=0.25$ and the service rate selected is $\mu=10$ requests/sec. To ensure stability of the system, we choose $\lambda_{n e t_{1}}$ and $\lambda_{n e t_{2}}$ such that,

$$
\lambda_{n e t_{1}}+\lambda_{\text {net }_{2}}<\mu
$$

By setting, $\lambda_{c}=\lambda_{\text {net }_{1}}=\lambda_{\text {net }_{2}}$, we present the average waiting times in Figure 6.12 for $0<\lambda_{c}<5$ requests/sec.

Figure 6.12 illustrates that the average waiting time for high priority requests is significantly reduced over both the scenarios of no priority and low priority requests. The average waiting time for high priority requests is calculated by considering the 
average waiting times while a single cluster is the only high priority cluster, as well as while both the UWB and Zigbee clusters are high priority. This is due to the fact that priority is assigned independently and both the UWB and Zigbee clusters are high priority with probability $p_{h}^{2}$; in the case where both clusters are high priority, the average waiting time is equal to the waiting time of the no priority scenario. This was derived in Chapter 5. A single cluster is the only high priority cluster with probability $p_{h}\left(1-p_{h}\right)$. Likewise, a cluster is low priority with probability $\left(1-p_{h}\right)\left(p_{h}\right)$.

The no priority scenario occurs with probability $\left(1-p_{h}\right)\left(1-p_{h}\right)$ and considers the case where no high priority traffic exists. The average waiting time for the no priority scenario is less than that for low priority traffic. The average waiting time is determined via total probability, as the weighted sum of the waiting times based on the probabilities for the four scenarios.

\subsection{Summary}

This chapter presented a performance evaluation of our cross-layer optimization algorithm. Our goal in designing this algorithm is to tie physical resource consumption to both throughput and energy capacity for WSNs. This is performed with the aim of maximizing performance benefits while achieving a predictable network lifetime.

In Chapter 5, we presented our expectation of the simulation. We desired to meet throughput performance listed in Table 1.1, while also testing our NPP to meet a one week network lifetime based on the required and applied energy capacities in Table 
5.3. From the performance evaluation presented, we were successful in meeting our throughput and network lifetime requirements.

We also presented a performance comparison of the UWB and Zigbee clusters in distributed WSNs. We evaluated our system based on performance metrics of total network throughput and mean per-hop throughput, for which we observed a significant gain achicved by our UWB cluster. This gain was also achieved in terms of spectral efficiency, which is intuitive given that UWB and Zigbee are high rate and low rate technologies, respectively. In terms of network lifetime, the UWB cluster managed to survive for two hours after the Zigbee network had lost full connectivity based on the initial capacities selected during system design.

Another significant result that was determined was the tightly coupled relationship between the blocking probability and the number of operating channels in the mesh network. This relationship is critical in determining the desired operating point in the network for the blocking probability. We confirmed that, because the mesh network supports transmissions from both the UWB and Zigbee clusters, it is quickly unable to support additional transmissions if the number of channels is low. Hence, in performing network planning, it is critical to pay careful attention to the number of channels that are selected for the mesh network during system design.

We also presented a priority scheme to handle critical sensor cluster transmissions. We utilize two priority levels such that high priority requests preempt active transmissions and jump to the front of the queue for cross-layer optimization. We compared the performance of the average waiting time for high priority requests with that of 
low priority requests. We also compared the performance to the scenario where no high priority requests exist in the network. We present a significant improvement in the average waiting time for these high priority requests.

The performance gains presented in this chapter are attainable due to our crosslayer optimization policy that is able to dynamically select paths to support energyaware routing algorithms, perform dynamic frequency selection to avoid channels with high interference, as well as adjust transmit power based on the power costs associated with transmitting at a higher transmission rate. The NPP enables us to achieve these performance gains, while meeting our desired network lifetime objective. While these results were shown for UWB, Zigbee and WiMax, our algorithm is not tied to any particular communication protocol. 


\section{Chapter 7}

\section{Conclusion}

This thesis addresses the throughput and energy optimization problem using crosslayer design in application-centric and distributed WSNs. Application-centric WSNs are those whose applications have significant performance requirements, but major considerations must also be made for energy conservation. Based on a literature review of joint throughput and energy optimization in WSNs, current approaches fall into one of two categories: those that perform rate control but do not model power cost in terms of the achievable throughput on the transmission link, or those that do not separate performance-related benefits and energy-related costs to ensure that we can jointly maximize throughput and minimize energy consumption.

Given these shortcomings, we present a cross-layer policy for joint throughput and energy optimization in these networks that is based on our link suitability method. The link suitability determines the optimal PHY layer resource allocation that jointly

maximizes achievable throughput and minimizes the associated power cost of using 
the link. By maximizing link suitability, our method ensures that WSNs allocate limited network resources to transmission channels capable of a higher level of throughput and energy conservation. By using link suitability in a cross-layer policy, we create a cross-layer optimization protocol that is unique in identifying routing paths with the optimal trade-off between throughput benefits and energy costs across multiple transmission channels. Our policy is based on transmit power at the PHY layer, channel allocation at the link layer and multi-hop path selection at the network layer. The NPP works in conjunction with our cross-layer optimization algorithm to enable us to study the impact of various network design decisions on our performance and network lifetime objectives. The relationship is derived between the expected performance of the communication protocol of interest, desired network lifetime, message load for data and coordination packets, and energy capacity.

These contributions have been evaluated in a distributed WSN architecture that is enabled by wireless mesh networks and uses a cross-layer optimization platform to revolutionize system control and resource management. The deployment of the distributed WSN enables us to position multiple sensor clusters geographically in various sensory locations of interest. These sensor clusters are able to gather sensory data for any number of high-bandwidth applications and feedback that information via the mesh backbone to a processing center for analysis. These clusters may be used for a diverse range of applications, from monitoring the health of building and bridge infrastructure for preemptive repairs, adjusting temperature and light levels in one or more rooms or building floors, and monitoring chemical levels in factories, 
for example. Other application include target tracking and video monitoring, among others. The use of the mesh network enables sensors to focus primarily on sensing and coordination functions with a reduced emphasis on multi-hop sensor routing. By only requiring that traffic is routed to the mesh node assigned to govern the cluster, this has the effect of conserving limited sensor energies in relaying traffic.

This routing requirement of only sending data to the cluster-head also has the effect of enabling short-range and high capacity communication protocols such as UWB to be used in dense sensor networks. Requiring that traffic reaches the clusterhead within a reasonable maximum hop count, such as four in this study, reduces UWB's reliance on multi-hop transmissions. These high speed technologies provide the opportunity to revolutionize system control and resource management through an expansion of the range of sensory applications for which these networks can be used. However, the cross-layer policy derived in this study is independent of the selection of any particular communication protocol and may be used in a variety of network configurations to achieve joint throughput and energy optimization.

The mesh network allows for nodes with greater resources to be responsible for executing our cross-layer optimization policy such that simple sensors are not expected to carry out higher level functions in the network. However, it should be noted that the deployed mesh network over a campus-sized area may be used to carry multiple types of traffic, only one of which may be sensory data. Thus, it should be considered that the results presented by the NPP only provide the necessary energy capacities to carry sensory traffic. Another benefit of the mesh network is that it expands 
connectivity to areas where fibre may not be available, hence the reason why mesh communication protocols and not IP is used in this thesis.

Our performance evaluation aims to prove that our cross-layer policy and NPP are able to jointly achieve significant throughput performance and desired network lifetimes for next-generation WSNs. In Chapter 5, we derive the required and applied initial energy capacities in Table 5.3 via the NPP that satisfy our throughput performance and network lifetime objectives. The NPP enables us to determine the necessary coordination protocols and perform system design to ensure reasonable energy capacities, while planning to meet the expected performance levels specified in Table 1.1 and a one week network lifetime. As a result, the NPP enables us to make network design decisions that meet network objectives in the presence of critical sensor network constraints. In terms of the performance of our sensor clusters, we present a significant improvement in the capacity achieved by UWB over Zigbee. In terms of spectral efficiency, UWB also provides more efficient use of the frequency spectrum in carrying a higher information rate. The performance evaluation also demonstrates that both clusters were able to survive for a desirable network lifetime of one week. These results point to the success of our solution to build next-generation WSNs.

The performance evaluation provides two additional results. First, we present the relationship between the blocking probability and the number of operating channels. We determine that the number of operating channels in the mesh network is critical to determining the operating point of the network. This is intuitive as the mesh network is shared between multiple sensor clusters with a greater demand for mesh 
network resources. Lastly, we present a priority scheme for distributed WSNs that enables the processing center to assign one or more clusters as high priority in the event that sensory data from these clusters requires an immediate response. High priority requests follow a preemptive resume scheme and shall preempt low priority transmissions such that request optimization is given the full view of mesh resources. We present a significant reduction in average waiting time for high priority requests given our priority algorithm.

\subsection{Practical Simplifications to Reduce Time Complexity}

In this thesis, a cross-layer policy for throughput and energy optimization in application-centric WSNs is presented. However, based on the specific application requirements for the network and different network configurations, some practical simplifications may be performed to reduce the complexity of the algorithm:

- Reduce the number of channels used in the network over which the cross-layer optimization and resource allocations are performed. However, one should consider that the resources in the mesh network are shared between multiple clusters and this should factor into the system design process;

- Enforce a single operating channel end-to-end across an entire path. This would reduce the complexity of the computation as permutations of channel allocations 
per-hop in Section 4.5.1 are not required. However, it would also increase the delay of the transmission as packets must be queued at each hop;

- Use machine learning algorithms to weight paths through feedback to affect the probability of selection. In this manner, more suitable neighbors of a source sensor are selected and the number of paths to evaluate may be reduced;

- Determine the spatial correlation between sensor measurements to reduce the number of transmission requests that require servicing from a single section of the sensor network; and,

- Replenish energy reserves for nodes in the mesh network to simplify the selection of mesh nodes in a path. This can be achieved through the use of solar or light sources, or access of mesh nodes to the power grid, if available. This would lead to negligible power costs of using the mesh node due to unlimited energy resources for the majority of the simulation. As mentioned, mesh nodes with energy reserves that are constantly replenished will be favored in the optimization algorithm over nodes whose energy reserves are constantly depleting.

\subsection{A Discussion on Software and Cognitive Radio}

A significant portion of this work requires the application of software and cognitive radio that we used to design state-of-the-art WSNs. Software radio is needed in our multi-technology system to convert operating parameters between heterogeneous 
technologies. This process was observed at the cluster-head where transmissions require conversion from sensor technologies such as UWB and Zigbee to mesh technologies such as WiMax. Cognitive radio, on the other hand, is used to provide sensors with spectrum sensing capabilities such that WSNs are able to operate around current environmental conditions by measuring the surrounding environment. However, both software and cognitive radios are not widely available in the market at the time of writing this thesis and, as a result, alternatives must be discussed.

One alternative for software radio which has been explored initially in a test bed is the use of a bridge available from Cisco that provides communication between Zigbee and WiFi. WiFi provides a shorter range than WiMax and is still suitable for distributed WSNs, although several additional mesh nodes would be required. As mentioned in Chapter 1, the 802.11s Task Group closed the Second Recirculation Sponsor Ballot in March 2011 with 95\% approval rating and is moving on to the Third Recirculation Sponsor Ballot [17]. Thus, while Zigbee does not attain high throughputs for application-centric WSNs, we may still enjoy the benefits of distributed WSNs albeit it for a smaller range of applications. UWB is also a viable technology and may be implemented by any communication protocol that follows its operating requirements. However, the design of UWB devices is complex given the need to design transceivers to operate over a wide band. Bluetooth LE is another option as it provides interoperability with $\mathrm{WiFi}$ and also attains data rates of $1 \mathrm{Mbps}$ [19]. However, the issue of synchronous communication needs to be resolved for Bluetooth LE to be suitable for WSN applications. Our cross-layer policy applies to any 
of these technologies and only requires adjustments to be made for the operating parameters discussed in Chapter 5. The energy efficiencies of the devices of these technologies must also be identified and applied.

For cognitive radio, there are a few alternatives that can be used. Firstly, given that the cluster-head is performing centralized resource allocation, the cluster-head can perform channel scheduling to ensure non-overlapping channels. This reduces cochannel interference by preventing multiple nearby sensors from transmitting over the same channel. However, without spectrum measurements, it will be difficult to avoid surrounding interference with sources outside of the sensor network. One solution may be to create a profile of operating channels in the spectrum band such that cluster-heads can keep track of channels that have high failure rates through feedback. This would not provide cluster-heads with surrounding interference measurements but provides additional information regarding which channels to avoid in the optimization process.

\subsection{Contributions}

To summarize, we have presented the following contributions of this research:

1. The link suitability method to quantify the optimal PHY layer resource allocation that achieves joint throughput and energy optimization in applicationcentric WSNs. By maximizing the trade-off between achievable throughput benefits and the associated power cost of using the link, we ensure that nodes 
are allocating limited resources optimally to transmission channels that are capable of a higher level of throughput and energy conservation;

2. A cross-layer optimization policy that is unique in identifying routing paths in WSNs with the optimal trade-off between throughput benefits and energy costs across multiple transmission channels. Our policy is based on: i) path selection at the network layer; ii) channel selection at the link layer; and, iii) transmit power based on the link suitability at the PHY layer; and,

3. A NPP that contributes the relationship between expected performance, network lifetime, message load and energy capacity for application-centric WSNs. The NPP enable network designers to study the impact of various network design decisions on performance and network lifetime objectives.

\subsection{Future Work}

Many areas exist for which the throughput and energy optimization problem may be extended. They will be explored in this section.

\subsubsection{Modulation, Bandwidth and Application Factors}

In this thesis, application performance was studied from the perspective of achievable throughput only. It shall be important to extend application-related factors to include delay and BER as these are also affected by resource allocation. In terms of 
additional PHY layer considerations, our cross-layer optimization algorithm should be extended to include modulation and bandwidth parameters in addition to current considerations of transmit power and frequency. It shall be important to study their impact on energy efficiency, objective function convergence, and meeting both performance and network lifetime objectives.

\subsubsection{Sensor Localization}

In this thesis, we consider sensors that are equipped with GPS tracking such that they are aware of their latitude and longitude positions. While manufacturers such as Garmin are making GPS sensors available, it is likely that it will be many years before sensors equipped with GPS are made available with reasonable sizes and costs for a diverse range of applications. We suspect that, in the next 3 to 5 years, sensor technology will have advanced enough such that sensors are able to perform selflocalization with GPS adapters at reasonable costs and sizes. Thus, in the mean time, methods for localization of sensor nodes are required.

Localization is required to execute the SPP protocol and determine available paths, as well as for optimization of requests to find the optimal path. Thus, while sensors with GPS capabilities are not available for application-centric WSNs, it may be required to deploy a third tier of nodes in the network, known as anchors, that would exist in a hierarchy below mesh nodes but above sensors in a cluster. These nodes, for which positions are known either through GPS or having been deployed in 
positions with known coordinates, are scattered throughout the sensor field to perform localization during the initialization phase. Several techniques for localization using anchors in WSNs are presented in [111]. Optimal placement of these nodes for maximum network lifetime or minimum energy consumption should also be explored. Given their availability, these anchors may also perform data aggregation or data correlation to reduce the number of routed messages in the network.

\subsubsection{Application Queries and Addressing}

It is likely that, in next-generation WSNs, sensors will be identified by applicationrelated queries, such as which nodes meet specific thresholds of temperature, light or chemical levels, for example. These queries would be forwarded through the network to retrieve critical data from individual sensors rather than requiring periodic data delivery from an entire sensor cluster. This addressing scheme complicates the cross-layer optimization process and its existing addressing scheme for sensors. Compatibility with these systems must be explored and it may be required for each sensor to both maintain a fixed address and respond to application-related queries.

\subsubsection{Back-Up Mesh Node Scheduling}

The deployment of redundant mesh nodes in our distributed WSN as proposed in this thesis raises additional research problems. Firstly, mesh nodes and their backups may take turns being active in order to balance energy consumption. Thus, 
optimal scheduling algorithms should be explored to determine wake and sleep cycles and should also consider variations in traffic intensity. Secondly, for multiple clusterheads used to service a single cluster, the optimal locations of these cluster-heads should be explored for maximum coverage and connectivity. 


\section{List of References}

[1] A. Chehri, P. Fortier, and P. Tardif, "Cross-layer link adaptation design for UWB-based sensor networks," Computer Communzcatıons, vol. 32, no. 13-14, pp. 1568-1575, 2009.

[2] I. Oppermann, L. Stoica, A. Rabbachin, Z. Shelby, and J. Haapola, "UWB wireless sensor networks: UWEN-a practical example," Communıcatıons Magazıne, IEEE, vol. 42, no. 12, pp. S27-S32, 2004.

[3] K. Yu and I. Oppermann, "UWB positioning for wireless embedded networks," in 2004 IEEE Radıo and Wireless Conference, pp. 459-462, 2004.

[4] V. Mehta and M. El Zarki, "An ultra wide band (uwb) based sensor network for civil infrastructure health monitoring," Work in Progress Session, EWSN, 2004.

[5] R. Madan, S. Cui, S. Lal, and A. Goldsmith, "Cross-layer design for lifetime maximization in interference-limited wireless sensor networks," Wireless Communzcatıons, IEEE Transactıons on, vol. 5, no. 11, pp. 3142-3152, 2006.

[6] E. Krogsveen, C. Wang, G. Oien, and S. Lindfors, "Energy-efficient adaptive route configuration in short-range wireless ad-hoc networks," in Wireless Communication Systems, 200\%. ISWCS 200\%. 4th International Symposium on, pp. 292-296, IEEE, 2007.

[7] C. Wang, L. Yin, and G. Oien, "Adaptive route configuration for increased energy efficiency in wireless sensor networks," in Broadband Communıcatıons, 
Networks and Systems, 2008. BROADNETS 2008. 5th Internatıonal Conference on, pp. 16-23, IEEE, 2009.

[8] X. Yin, X. Zhou, Z. Li, and S. Li, "Cross-Layer Based Rate Control for Lifetime Maximization in Wrreless Sensor Networks," Advances in Grıd and Pervasıve Computing, pp. 140-149, 2010.

[9] D. Kim, K. Shazzad, and J. Park, "A framework of survivability model for wireless sensor network," in Proc. of Int. Conf. on Avallabılıty, Relıabılıty and Securty, pp. 515--522, 2006.

[10] H. Botee and E. Bonabeau, "Evolving ant colony optimization," To appear in Advances in Complex Systems, vol. 1, no. 2/3, pp. 149-159, 1999.

[11] A. Colorni, M. Dorigo, V. Maniezzo, et al., "Distributed optimization by ant colonies," in Proceedıngs of the First European Conference on Artıficıal Life, pp. 134-142, 1991.

[12] Y. Liu and K. Passino, "Swarm intelligence Literature overview," Dept. of Electrical Engineering, The Ohıo State Unıversity, vol. 2015.

[13] R. Vullers, R. Schaijk, H. Visser, J. Penders, and C. Hoof, "Energy harvesting

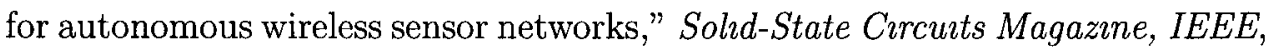
vol. 2, no. 2, pp. 29-38, 2010.

[14] G. Martin, "Wireless Sensor Solutions for Home \& Building Automation- The Successful Standard Uses Energy Harvesting," EnOcean@ White Papers, Nov, 2006.

[15] R. Bruno, M. Contı, and E. Gregori, "Mesh networks: commodity multihop ad hoc networks," IEEE Communications Magazıne, vol. 43, no. 3, pp. 123-131, 2005.

[16] "Status of Project IEEE 802.11s- ESS Mesh Networking," tech. rep., IEEE P802.11 Task Group S, March 2011. http://www.ieee802.org/11/Reports/ tgs_update.htm. 
[17] "802.11 Liaison Report for 2011-03," tech. rep., Clint Chaplin, $802.11 \mathrm{Li}-$ aison (Samsung), March 2011. https://mentor.ieee.org/802.21/dcn/11/ 21-11-0059-00-0000-802-11-1iaison-report-for-2011-03.ppt.

[18] "A Technology Comparison: Adopting Ultra-Wideband for Memsen's file sharing and wireless marketing platform," tech. rep., Memsen Corporation, May 2004. http://wireless.fcc.gov/outreach/2004broadbandforum/ comments/ultrawideband.pdf.

[19] Bluetooth Special Interest Group, Bluetooth Specification Version 4.0, June 2010. https://www.bluetooth.org/Technical/Specificatıons/adopted. htm.

[20] S. Cheekiralla and D. Engels, "A functional taxonomy of wireless sensor network devices," Broadband Networks, 2005 2nd International Conference on, pp. 2633,2005 .

[21] N. Malakooty, "Ultra Wideband Technology and the Struggle to Adopt a Standard for the Consumer Electronics Industry," Personal Computing Industry Center, p. 384, 2006.

[22] IEEE 802.15.3b-2005, Part 15.3: Wreless Medium Access Control (MAC) and Physıcal Layer (PHY) Specıfications for High Rate Wireless Personal Area Networks (WPANs) Amendment 1: MAC Sublayer, May 2006.

[23] J. Baliga, R. Ayre, W. Sorin, K. Hinton, and R. Tucker, "Energy consump-

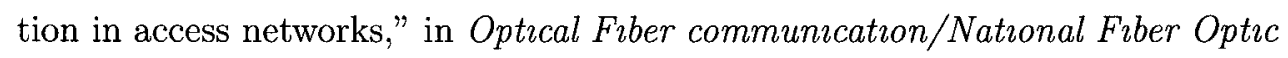
Engineers Conference, 2008. OFC/NFOEC 2008. Conference on, pp. 1-3, 2008.

[24] J. Ploennigs, U. Ryssel, and K. Kabitzsch, "Performance analysis of the EnOcean wireless sensor network protocol," in Emerging Technologies and Factory Automation (ETFA), 2010 IEEE Conference on, pp. 1-9, IEEE. 
[25] B. Zhang, R. Simon, and H. Aydin, "Energy management for time-critical energy harvesting wireless sensor networks," Stabılızatıon, Safety, and Securıty of Distributed Systems, pp. 236--251, 2010.

[26] T. Zhu, Z. Zhong, Y. Gu, T. He, and Z. Zhang, "Leakage-aware energy synchronization for wireless sensor networks," in Proceedings of the 7th internatıonal conference on Mobule systems, applıcatıons, and services, pp. 319-332, ACM, 2009.

[27] D. Hasenfratz, A. Meıer, C. Moser, J. Chen, and L. Thiele, "Analysis, Comparison, and Optimization of Routing Protocols for Energy Harvesting Wireless Sensor Networks," in 2010 IEEE International Conference on Sensor Networks, Ubrqurtous, and Trustworthy Computıng, pp. 19-26, IEEE, 2010.

[28] W. Wang, T. O'Donnell, L. Ribetto, B. O'Flynn, M. Hayes, and C. O'Mathuna, "Energy harvesting embedded wireless sensor system for building environment applications," in Wireless Communıcatıon, Vehıcular Technology, Information Theory and Aerospace \& Electronuc Systems Technology, 2009. Wireless VITAE 2009. 1st International Conference on, pp. 36-41, IEEE, 2009.

[29] Y. Shi, Y. Hou, H. Sherali, and S. Midkiff, "Optimal routing for UWB-based sensor networks," IEEE Journal on Selected Areas in Communıcatıons, vol. 24, no. 4 , pp. 857-863, 2006.

[30] M. Bahr, "Proposed routing for IEEE 802.11 s WLAN mesh networks," in Proceedings of the 2nd annual international workshop on Wireless internet, ACM New York, NY, USA, 2006.

[31] V. Srivastava and M. Motani, "Cross-layer design: a survey and the road ahead," Communicatıons Magazıne, IEEE, vol. 43, no. 12, pp. 112-119, 2005.

[32] Q. Wang and M. Abu-Rgheff, "Cross-layer signalling for next-generation wireless systems," in IEEE WCNC, vol. 2, pp. 1084-89, Citeseer, 2003. 
[33] Z. In and B. Lf, "Fundamental performance limits of wireless sensor networks," Ad-Hoc and Sensor Networks: Wireless Networks and Mobıle Computıng, p. 91, 2005 .

[34] C. Peraki and S. Servetto, "On the maximum stable throughput problem in random networks with directional antennas," in Proceedings of the 4th ACM internatıonal symposium on Mobıle ad hoc networking \& computıng, pp. 76-87, ACM New York, NY, USA, 2003.

[35] P. Gupta and P. Kumar, "The capacity of wireless networks," IEEE Transactıons on informatıon theory, vol. 46, no. 2, pp. 388-404, 2000.

[36] S. Arms, C. Townsend, D. Churchill, M. Hamel, J. Galbreath, and S. Mundell, "Frequency Agile Wireless Sensor Networks," Proc. SPIE Int. Socıety for Optıcal Engineerıng, vol. 5389, pp. 468-475, 2004.

[37] J. Galbreath and J. Frolik, "Channel allocation strategies for wireless sensors statically deployed in multipath environments," in Proceedings of the 5th international conference on Information processing in sensor networks, pp. 334-341, ACM New York, NY, USA, 2006.

[38] D. Churchill, M. Hamel, C. Townsend, and S. Arms, "Miniaturized wireless inertial sensing system," Mar. 2 2010. US Patent 7,672,781.

[39] G. Anastasi, M. Conti, M. Di Francesco, and A. Passarella, "Energy conservation in wireless sensor networks: A survey," Ad Hoc Networks, vol. 7, no. 3, pp. 537-568, 2009.

[40] J. Crichigno, M. Wu, S. Jayaweera, and W. Shu, "Throughput Optimization in Multi-hop Wireless Networks with Multi-packet Reception and Directional Antennas," IEEE Transactions on Parallel and Distrıbuted Systems, 2010.

[41] D. ONeill, D. Julian, and S. Boyd, "Seeking Foschinis genie: optimal rates and powers in wireless networks," IEEE Transactıons on Vehıcular Technology, 2004 . 
[42] H. Wang, D. Peng, W Wang, H. Sharif, and H. Chen, "Cross-layer routing optimization in multirate wireless sensor networks for distributed source coding based applications," Wireless Communicatıons, IEEE Transactıons on, vol. 7, no. 10, pp. 3999-4009, 2008.

[43] L. Luo, Y. Bai, and W. Wu, "A Novel Energy Optimizatıon Approach for Wireless Sensor Networks," Communıcation and Networkıng, pp. 226-233, 2009.

[44] D. Ferrara, L. Galluccio, A. Leonardi, G. Morabito, and S. Palazzo, "MACRO: an integrated $\mathrm{MAC} /$ routing protocol for geographic forwarding in wireless sensor networks," in INFOCOM 2005. 24th Annual Jount Conference of the IEEE

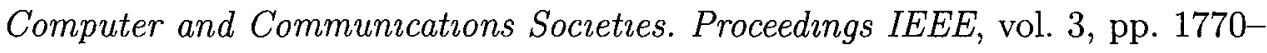
1781, IEEE, 2005.

[45] G. Zussman and A. Segall, "Energy efficient routing in ad hoc disaster recovery networks," Ad Hoc Networks, vol. 1, no. 4, pp. 405-421, 2003.

[46] A. Sankar and Z. Liu, "Maximum lifetıme routing in wireless ad-hoc networks," in INFOCOM 2004. Twenty-thurd Annual Joint Conference of the IEEE Computer and Communicatıons Socıetıes, vol. 2, pp. 1089-1097, IEEE, 2004.

[47] J. Chang and L. Tassiulas, "Energy conserving routing in wireless ad-hoc networks," in INFOCOM 2000. Nineteenth Annual Joint Conference of the IEEE Computer and Communications Societıes. Proceedings. IEEE, vol. 1, pp. 22-31, IEEE, 2002.

[48] R. Yu, Y. Zhang, L. Song, and W. Yao, "Joint energy and route optimization in MIMO Wireless Sensor Networks," in Wireless Internet Conference (WICON), 2010 The 5th Annual ICST, pp. 1-6, IEEE, 2010.

[49] C. Chong and S. Kumar, "Sensor networks: Evolution, opportunities, and challenges," Proceedıngs of the IEEE, vol. 91, no. 8, pp. 1247-1256, 2003. 
[50] M. Vuran and I. Akyildiz, "Spatial correlation-based collaborative medium access control in wireless sensor networks," IEEE/ACM Transactıons on Networkıng (TON), vol. 14, no. 2, pp. 316-329, 2006.

[51] W. Heinzelman, A. Chandrakasan, and H. Balakrishnan, "Energy-efficient communication protocol for wireless microsensor networks," in System Scrences, 2000. Proceedings of the 33rd Annual Hawan International Conference on, p. 10, IEEE, 2002.

[52] D. Baker and A. Ephremides, "The architectural organization of a mobile radio network via a distributed algorithm," Communıcatıons, IEEE Transactıons on, vol. 29, no. 11, pp. 1694-1701, 2002.

[53] B. Das and V. Bharghavan, "Routing in ad-hoc networks using minimum connected dominating sets," in Communıcatıons, 1997. ICC 97 Montreal, 'Towards the Knowledge Millennıum' 1997 IEEE Internatıonal Conference on, vol. 1, pp. 376-380, IEEE, 2002.

[54] S. Bandyopadhyay and E. Coyle, "An energy efficient hierarchical clustering algorithm for wireless sensor networks," in INFOCOM 2003. Twenty-Second Annual Joint Conference of the IEEE Computer and Communications. IEEE Socıetıes, vol. 3, pp. 1713-1723, IEEE, 2003.

[55] C. Lin and M. Gerla, "Adaptive clustering for mobile wireless networks," Selected Areas in Communications, IEEE Journal on, vol. 15, no. 7, pp. 1265$1275,2002$.

[56] A. Amis, R. Prakash, T. Vuong, and D. Huynh, "Max-min d-cluster formation in wireless ad hoc networks," in INFOCOM 2000. Nineteenth Annual Joint Conference of the IEEE Computer and Communıcatıons Socıetıes. Proceedings. IEEE, vol. 1, pp. 32-41, IEEE, 2002.

[57] I. Akyildiz and E. Stuntebeck, "Wireless underground sensor networks: Research challenges," Ad Hoc Networks, vol. 4, no. 6, pp. 669-686, 2006. 
[58] K. Lorincz, D. Malan, T. Fulford-Jones, A. Nawoj, A. Clavel, V. Shnayder, G. Mainland, M. Welsh, and S. Moulton, "Sensor networks for emergency response: Challenges and opportunities," Pervasive Computing, IEEE, vol. 3, no. 4, pp. 16-23, 2005.

[59] L. Schwiebert, S. Gupta, and J Weinmann, "Research challenges in wireless networks of biomedical sensors," in Proceedings of the 7th annual international conference on Mobile computıng and networking, pp. 151-165, ACM, 2001.

[60] H. Zhou, D. Luo, Y. Gao, and D. Zuo, "Modeling of Node Energy Consumption for Wireless Sensor Networks," Wireless Sensor Network, vol. 3, no. 1, pp. 18$23,2011$.

[61] A. Chamam and S. Pierre, "On the planning of wireless sensor networks: Energy-efficient clustering under the joint routing and coverage constraint," IEEE Transactions on Mobıle Computıng, pp. 1077-1086, 2009.

[62] J. Gao, "Analysis of energy consumption for ad hoc wireless sensor networks using a bit-meter-per-joule metric," IPN Progress Report, vol. 42, p. 150, 2002.

[63] G. Mergen, Q. Zhao, and L. Tong, "Sensor networks with mobile access: Energy and capacity considerations," Communıcatıons, IEEE Transactıons on, vol. 54, no. 11, pp. 2033-2044, 2006.

[64] V. Tsiatsis, S. Zimbeck, and M. Srivastava, "Architecture strategies for energyefficient packet forwarding in wireless sensor networks," in Proceedings of the 2001 international symposıum on Low power electronıcs and desıgn, pp. 92-95, ACM New York, NY, USA, 2001.

[65] W. Heinzelman, J. Kulik, and H. Balakrishnan, "Adaptive protocols for information dissemination in wireless sensor networks," Proceedings of the 5th annual ACM/IEEE international conference on Mobile computing and networking, pp. 174-185, 1999. 
[66] J. Kulik, W. Heinzelman, and H. Balakrishnan, "Negotiation-Based Protocols for Disseminating Information in Wireless Sensor Networks," Wireless Networks, vol. 8, no. 2, pp. 169-185, 2002.

[67] C. Intanagonwiwat, R. Govindan, and D. Estrin, "Directed diffusion: A scalable and robust communication paradigm for sensor networks," Proceedings of the ACM/IEEE International Conference on Mobile Computing and Networking, pp. 56-67, 2000.

[68] J. Al-Karaki and A. Kamal, "Routing techniques in wireless sensor networks: a survey," Wireless Communications, IEEE [see also IEEE Personal Communıcatıons], vol. 11, no. 6, pp. 6-28, 2004.

[69] D. Braginsky and D. Estrin, "Rumor routing algorthim for sensor networks," Proceedings of the 1st ACM international workshop on Wireless sensor networks and applecatıons, pp. 22-31, 2002.

[70] F. Ye, A. Chen, S. Lu, and L. Zhang, "A scalable solution to minimum cost forwarding in large sensor networks," in Computer Communicatıons and Networks, 2001. Proceedings. Tenth International Conference on, pp. 304-309, IEEE, 2002.

[71] Y. Yao and J. Gehrke, "The cougar approach to in-network query processing in sensor networks," SIGMOD record, vol. 31, no. 3, pp. 9-18, 2002.

[72] N. Sadagopan, B. Krishnamachari, and A. Helmy, "The ACQUIRE mechanism for efficient querying in sensor networks," in Sensor Network Protocols and Applications, 2003. Proceedings of the First IEEE. 2003 IEEE International Workshop on, pp. 149-155, IEEE, 2003.

[73] K. Akkaya and M. Younis, "A survey on routing protocols for wireless sensor networks," Ad Hoc Networks, vol. 3, no. 3, pp. 325-349, 2005.

[74] R. Shah and J. Rabaey, "Energy aware routing for low energy ad hoc sensor networks," Wireless Communications and Networking Conference, 2002. WCNC2002. 2002 IEEE, vol. 1, 2002. 
[75] M. Hasegawa, T. Kawamura, H. Tran, G. Miyamoto, Y. Murata, H. Harada, and S. Kato, "Decentralized optimization of wireless sensor network lifetime based on neural network dynamics," in Personal, Indoor and Mobıle Radıo Communications, 2008. PIMRC 2008. IEEE 19th International Symposium on, pp. 1-5, IEEE, 2008.

[76] J. Chang and L. Tassiulas, "Maximum lifetime routing in wireless sensor networks," IEEE/ACM Transactions on Networking (TON), vol. 12, no. 4, pp. 609-619, 2004.

[77] J. Chang and L. Tassiulas, "Energy conserving routing in wireless ad-hoc networks," in IEEE INFOCOM 2000. Nineteenth Annual Jount Conference of the IEEE Computer and Communıcatıons Socıetıes. Proceedıngs, vol. 1, 2000.

[78] D. Ganesan, R. Govından, S. Shenker, and D. Estrin, "Highly-resilient, energyefficient multipath routing in wireless sensor networks," ACM SIGMOBILE Mobıle Computıng and Communıcatıons Revıew, vol. 5, no. 4, pp. 11-25, 2001.

[79] A. Amis and R. Prakash, "Load-balancing clusters in wireless ad hoc networks," in Application-Specific Systems and Software Engineering Technology, 2000. Proceedıngs. 3rd IEEE Symposium on, pp. 25-32, IEEE, 2002.

[80] M. Chatterjee, S. Das, and D. Turgut, "WCA. A weighted clustering algorithm for mobile ad hoc networks," Cluster Computıng, vol. 5, no. 2, pp. 193-204, 2002 .

[81] S. Basagni, "Distributed and mobility-adaptive clustering for multimedia support in multi-hop wireless networks," in Vehıcular Technology Conference, 1999. VTC 1999-Fall. IEEE VTS 50th, vol. 2, pp. 889-893, IEEE, 2002.

[82] S. Basagni, "Distributed clustering for ad hoc networks," in $2 s p a n$, p. 310, Published by the IEEE Computer Society, 1999.

[83] M. Gerla and J. Tsai, "Multicluster, mobıle, multimedia radio network," Wireless networks, vol. 1, no. 3, pp. 255-265, 1995. 
[84] A. McDonald and T. Znati, "A mobility-based framework for adaptive clustering in wireless ad hoc networks," Selected Areas in Communicatıons, IEEE Journal on, vol. 17, no. 8, pp. 1466-1487, 2002.

[85] C. Lin and M. Gerla, "Adaptive clustering for mobile wireless networks," IEEE Journal on Selected areas in Communicatıons, vol. 15, no. 7, pp. 1265-1275, 1997.

[86] A. Matin and S. Hussain, "Intelligent hierarchical cluster-based routing," Proceedings of the International Workshop on Mobility and Scalability in Wireless Sensor Networks (MSWSN) in IEEE International Conference on Distributed Computing in Sensor Networks (DCOSS), pp. 165-172, 2006.

[87] M. Ye, C. Li, G. Chen, and J. Wu, "EECS: An energy efficient clustering scheme in wireless sensor networks," in Performance, Computıng, and Communicatıons Conference, 2005. IPCCC 2005. 24th IEEE International, pp. 535-540, IEEE, 2005 .

[88] C. Chiasserini, I. Chlamtac, P. Monti, and A. Nucci, "Energy efficient design of wireless ad hoc networks," NETWORKING 2002: Networking Technologies, Services, and Protocols; Performance of Computer and Communicatıon Networks; Mobıle and Wireless Communicatıons, pp. 376-386, 2006.

[89] D. Estrin, R. Govindan, J. Heidemann, and S. Kumar, "Next century challenges: Scalable coordination in sensor networks," in Proceedings of the 5th annual ACM/IEEE international conference on Mobile computıng and networking, pp. 263-270, ACM, 1999.

[90] S. Chandrachood, A. Anthony, and T. Jannett, "Using resource based modeling to evaluate coordination schemes in wireless sensor networks," Proceedings of IEEE SoutheastCon, Memphrs, pp. 91-97, 2006.

[91] R. Ellison, D. Fisher, R. Linger, H. Lipson, T. Longstaff, and N. Mead, "Survivable network systems: An emerging discipline," Software Engıneering Instıtute, Carnegue Mellon University, 1997. 
[92] W. Wang and B. Li, "Market-driven bandwidth allocation in sclfish overlay networks," in Proceedings IEEE INFOCOM 2005. 24th Annual Jount Conference of the IEEE Computer and Communications Societıes, vol. 4, 2005.

[93] L. Xiao, M. Johansson, and S. Boyd, "Simultaneous routing and resource allocation via dual decomposition," IEEE Transactıons on Communıcatıons, vol. 52, no. 7 , pp. 1136-1144, 2004.

[94] Y. Sankarasubramaniam, I. Akyildiz, and S. McLaughlin, "Energy efficiency based packet size optimization in wireless sensor networks," Sensor Network Protocols and Applications, 2003. Proceedings of the First IEEE. 2003 IEEE International Workshop on, pp. 1-8, 2003.

[95] Y. Sankarasubramaniam, I. Akyildiz, and S. McLaughlin, "Energy efficiency based packet size optimization in wireless sensor networks," in Sensor Network Protocols and Applications, 2003. Proceedings of the First IEEE. 2003 IEEE Internatzonal Workshop on, pp. 1-8, IEEE, 2003.

[96] S. Cho and K. Kim, "Variable bandwidth allocation scheme for energy efficient wircless sensor network," Communıcatıons, 2005. ICC 2005. 2005 IEEE Internatıonal Conference on, vol. 5, 2005.

[97] R. Kershner, "The number of circles covering a set," Amerıcan Journal of Mathematrcs, vol. 61, no. 3, pp. 665-671, 1939.

[98] H. Zhang and J. Hou, "Maintaining Sensing Coverage and Connectivity in Large Sensor Networks," Ad Hoc \& Sensor Wireless Networks, vol. 14, p. 28, 2005.

[99] D. Tse and P. Viswanath, Fundamentals of wireless communıcatıon. Cambridge University Press, 2005.

[100] E. Staggs, "Ultrawideband Radio Design - System Analysis," in Partners in Desıgn, Worldwıde Technıcal Workshops 2004, 2004. 
[101] IEEE 802.16-2004, IEEE Standard for Local and Metropolitan Area Networks Part 16: Air Interface for Fixed Broadband Wireless Access Systems, October 2004.

[102] IEEE 802.15.4-2006, IEEE Standard for Local and Metropolatan Area Networks Part 15.4: Wireless Medium Access Control (MAC) and Physical Layer (PHY) Specufications for Low Rate Wireless Personal Area Networks (LR-WPANs), Scptember 2006.

[103] O. Sheikh and S. Mahmoud, "Throughput and Energy Optimization via CrossLayer Design in Distributed Wireless Sensor Networks," (submitted), 2011.

[104] E. Dijkstra, "A note on two problems in connexion with graphs," Numerısche mathematık, vol. 1, no. 1, pp. 269-271, 1959.

[105] O. Sheikh, O. Aboul-Magd, and S. Mahmoud, "Node Coordination for Centralized Control of Cross-Layer Optimization in Two-Tier Multi-Hop Wireless Sensor Networks," (submitted), 2011.

[106] O. Sheikh, O. Aboul-Magd, and S. Mahmoud, "Network Planning for Sustainable Network Lifetimes and Improved Application Performance in Distributed Wireless Sensor Networks," (submitted), 2011.

[107] J. Nelder and R. Mead, "A simplex method for function minimization," The computer journal, vol. 7, no. 4, p. 308, 1965.

[108] J. Lagarias, J. Reeds, M. Wright, and P. Wright, "Convergence properties of the Nelder-Mead simplex method in low dimensions," SIAM Journal on Optrmization, vol. 9, no. 1, pp. 112-147, 1999.

[109] K. McKinnon, "Convergence of the Nelder-Mead simplex method to a nonstationary point," SIAM Journal on Optımızatıon, vol. 9, no. 1, pp. 148-158, 1999.

[110] M. Barbcau, E. Kranakis, and E. Corporation, Princıples of ad hoc networkıng. Wiley Online Library, 2007. 
[111] G. Mao, B. Fidan, and B. Anderson, "Wireless sensor network localization techniques," Computer Networks, vol. 51, no. 10, pp. 2529-2553, 2007. 


\section{Appendix A}

\section{Summary of the Cross-Layer Optimization}

\section{Policy for Path, Channel, Power and Rate}

\section{Negotiation}

1. Initialization State \{network layer\}:

form simple path matrix $X$ for one cluster and mesh using the Simple Path Protocol (SPP).

Repeat for each sensor cluster deployed.

\{Repeat for all requests\}:

2. At time of request, extract path list $A \subset X$ for source node $v_{i}$ of transmission. Set initial path to evaluate, $a=1$, and done_already $\left(v_{x}, v_{y}\right)=0, \forall v_{x}, v_{y} \in Z$;

3. Select path $a$ to evaluate:

(a) If $a=1$ and path $a \notin A$ then simple path does not exist between $v_{i}$ and $v_{j}$ and there cannot be an optimal path. Network partitioning has occurred and request is rejected.

(b) If $a \neq 1$ and path $a \notin A$, then we have iterated over all paths. Go to Step 5 .

(c) If path $a \in A$ exists:

i. Link Optimization State $\{\mathrm{PHY}$ layer $\}$ :

optimize all hops in path $a$ by finding optimum link utilities $L_{v_{x}, v_{y}, f}^{*}$ over all transmitter-receiver pairs, i.e. $\forall v_{x}, v_{y} \in$ path $a$ :

A. Check if done_already $\left(v_{x}, v_{y}\right)=1$. If true, jump to Step 3(c)iC, else continue to Step 3(c)iB.

B. Optimize $L_{v_{x}, v_{y}, f}^{*}$ over all channels, $\forall f \in F$ using Equation (3.8) for $v_{x}-v_{y}$ pair using previous_solution $\left(v_{x}, v_{y}, f\right)$ as initial value using the Nelder-Mead method. 
C. Store $f, P_{v_{x}, f}^{t^{*}}, r_{v_{x}, v_{y}, f}^{*}$ and $L_{v_{x}, v_{y}, f}^{*}$.

D. Set previous_solution $\left(v_{x}, v_{y}, f\right)=P_{v_{x}, f}^{t^{*}}, \forall f \in F$.

E. Set done_already $\left(v_{x}, v_{y}\right)=1$.

ii. Channel Optimization State\{data link layer\}:

determine the receiver $v_{y}$ 's $C=5$ most preferred bins over all hops, $\forall v_{y} \in$ path $a$ :

A. Form Table 4.1 using $f$ and $L_{v_{x}, v_{y}, f}^{*}$ values from Step 3(c)iC.

B. Rank bins in order of $L_{v_{x}, v_{y}, f}^{*}$.

C. Select top $C=5$ bins from Step 3(c)iiB and form Table 4.2.

D. Iterate over $C^{K^{\prime}}$ possible bin combination assignments.

E. Select bin combination $f_{1}^{*}, \ldots, f_{K^{\prime}}^{*}$ that leads to largest valid profit sum, $U_{v_{i}, v_{j}}^{a}$, according to constraints in Equation (3.6).

F. Store $U_{v_{i}, v_{j}}^{a}$ and $f_{1}^{*}, \ldots, f_{K^{\prime}}^{*}$ for path $a$.

4. Set $a=a+1$ to select the next candidate path. Go back to step 3 .

5. Path Optimization State \{network layer\}:

determine optimal profit $\operatorname{sum} U_{v_{i}, v_{j}}^{a^{*}}$, bin allocation $f_{1}^{*}, \ldots, f_{K^{\prime}}^{*}$, transmit power $P_{v_{x}, f}^{t^{*}}$, rate $r_{v_{x}, v_{y}, f}^{*}$ and $L_{v_{x}, v_{y}, f}^{*}$ from set of candidate paths $A$.

(a) Retrieve profit sums, $\left\{U_{v_{v}, v}^{a}\right\}$, for all candidate paths $a \in A$ from 3(c)iiF.

(b) Select path $a^{*}$ with maximum profit sum, $U_{v_{i}, v_{j}}^{a^{*}}$ as optimal path.

(c) Retrieve stored bin allocation $f_{1}^{*}, \ldots, f_{K^{\prime}}^{*}$ for path $a^{*}$.

(d) Retrieve optimal link utilities $L_{v_{x}, v_{y}, f}^{*}$, rates $r_{v_{x}, v_{y}, f}^{*}$ and transmit power $P_{v_{x}, f}^{*}$ for each hop along $a^{*}$.

6. Transmission State $\left\{\mathrm{PHY}\right.$ layer\}: Inform all $v_{x}-v_{y}$ pairs along path $a^{*}$ of their next hop, required transmit powers $P_{1, f}^{t^{*}}, \ldots, P_{K^{\prime}, f}^{t^{*}}$, and channel selection $f_{1}^{*}, \ldots, f_{K^{\prime}}^{*}$.

7. Set done_already $\left(v_{x}, v_{y}\right)=0, \forall v_{x}, v_{y} \in Z$ for next request.

Figure A.1: Summary of the Cross-Layer Optimization Policy for Path, Channel, Power and Rate Negotiation 


\section{Appendix B}

\section{Link Budget Analysis for UWB, Zigbee and WiMax}

\begin{tabular}{cccc}
\hline Parameter & UWB & Zigbee & $802.16-2004 \mathrm{WiMax}$ \\
\hline $\begin{array}{c}\text { Information } \\
\text { Data Rate }\end{array}$ & $480 \mathrm{Mb} / \mathrm{s}$ & $250 \mathrm{~Kb} / \mathrm{s}$ & $75 \mathrm{Mb} / \mathrm{s}$ \\
\hline $\begin{array}{c}\text { Average Maximum } \\
\text { Transmit Power }\end{array}$ & $-14.3 \mathrm{dBm}$ & $0 \mathrm{dBm}$ & $11 \mathrm{dBm}$ \\
\hline $\begin{array}{c}\text { Total Path Loss } \\
\text { (@ max range) }\end{array}$ & $\begin{array}{c}60.7 \mathrm{~dB} \\
(10.6 \mathrm{~m})\end{array}$ & $\begin{array}{c}(0 \mathrm{~dB} \\
(8.9 \mathrm{~m})\end{array}$ & $\begin{array}{c}84.2 \\
\text { (1.04 km) }\end{array}$ \\
\hline Average Rx Power & $-75 \mathrm{dBm}$ & $-75 \mathrm{dBm}$ & $-73.2 \mathrm{dBm}$ \\
\hline Noise Power Per Bit & $-87 \mathrm{dBm}$ & $-120 \mathrm{dBm}$ & $-104 \mathrm{dBm}$ \\
\hline Rx Noise Figure & $0.60 \mathrm{~dB}$ & $33.2 \mathrm{~dB}$ & $8 \mathrm{~dB}$ \\
\hline Total Noise Power & $-86.4 \mathrm{dBm}$ & $-86.8 \mathrm{dBm}$ & $-96 \mathrm{dBm}$ \\
\hline Required E $/ N_{0}$ & $-1.59 \mathrm{~dB}$ & $-1.2 \mathrm{~dB}$ & $9.8 \mathrm{~dB}$ \\
\hline Implementation Loss & $3.0 \mathrm{~dB}$ & $3.0 \mathrm{~dB}$ & $3.0 \mathrm{~dB}$ \\
\hline Link Margin & $10 \mathrm{~dB}$ & $10 \mathrm{~dB}$ & $10 \mathrm{~dB}$ \\
\hline Rx Sensitivity & $-85 \mathrm{dBm}$ & $-85 \mathrm{dBm}$ & $-83.2 \mathrm{dBm}$ \\
\hline
\end{tabular}

Table B.1: A Link Budget Analysis for Zigbee, UWB and WiMax [100] [101] [102] 


\section{Appendix C}

\section{Proof of Convergence for $L_{v_{x}, v_{y}, f}^{*}$ and $U_{v_{i}, v_{j}}^{a^{*}}$}

To prove the convergence of our link utility function $L_{v_{x}, v_{y}, f}^{*}$ and objective function $U_{v_{x}, v_{3}}^{a^{*}}$ to the optimal solution, we form our link utility function as,

$$
\begin{aligned}
L_{v_{x}, v_{y}, f}^{*}\left(P_{v_{x}, f}^{t}, t\right)= & \alpha \log _{10}\left(1+\frac{w_{f} \log _{2}\left(1+\frac{P_{v_{x}, f}^{t}\left|H_{v_{x}, v_{y}, f}\right|^{2}}{w_{f}, v_{y_{y}, f}+I_{y_{y}, f}}\right)}{C_{v_{y}, f}(t)}\right)- \\
& \frac{\psi(E)}{m} q_{t_{v_{x}}} l w_{f} \log _{2}\left(1+\frac{P_{v_{x}, f}^{t}\left|H_{v_{x}, v_{y}, f}\right|^{2}}{w_{f} N_{v_{y}, f}+I_{v_{y}, f}}\right)+\eta
\end{aligned}
$$

and differentiate $-L_{v_{x}, v_{y}, f}^{*}\left(P_{v_{x}, f}^{t}, t\right)$ to yield,

$$
-\frac{\partial L_{v_{x}, v_{y}, f}^{*}}{\partial P_{v_{x}, f}^{t}}=\frac{w_{f}\left|H_{v_{x}, v_{y}, f}\right|^{2} \Omega\left(P_{v_{x}, f}^{t}, t\right)}{\left(w_{f} N_{v_{y}, f}+I_{v_{y}, f}+P_{v_{x}, f}^{t}\left|H_{v_{x}, v_{y}, f}\right|^{2}\right) \log (2) \log (10) \Phi\left(P_{v_{x}, f}^{t}, t\right)}
$$

where,

$$
\begin{aligned}
\Omega\left(P_{v_{x}, f}^{t}, t\right)= & w_{f} \frac{\psi(E) l}{m} q_{t_{x}} \log (10) \log \left(1+\frac{P_{v_{x}, f}^{t}\left|H_{v_{x}, v_{y}, f}\right|^{2}}{w_{f} N_{v_{y}, f}+I_{v_{y}, f}}\right)+ \\
& \log (2)\left(\frac{\psi(E) l}{m} q_{t_{v_{x}}} C_{v_{y}, f}(t) \log (10)-\alpha\right)
\end{aligned}
$$

and,

$$
\Phi\left(P_{v_{x}, f}^{t}, t\right)=\left(\log (2) C_{v_{y}, f}(t)+w_{f} \log \left(1+\frac{P_{v_{x}, f}^{t}\left|H_{v_{x}, v_{y}, f}\right|^{2}}{w_{f} N_{v_{y}, f}+I_{v_{y}, f}}\right)\right)
$$


Differentiating again, we obtain,

$$
-\frac{\partial^{2} L_{v_{x}, v_{y}, f}^{*}}{\partial P_{v_{x}, f}^{t}}=-\frac{w_{f}\left|H_{v_{x}, v_{y}, f}\right|^{4} \Delta\left(P_{v_{x}, f}^{t}, t\right)}{\left(w_{f} N_{v_{y}, f}+I_{v_{y}, f}+P_{v_{x}, f}^{t}\left|H_{v_{x}, v_{y}, f}\right|^{2}\right)^{2} \log (2) \log (10) \Phi\left(P_{v_{x}, f}^{t}, t\right)^{2}}
$$

where,

$$
\begin{aligned}
\Delta\left(P_{v_{x}, f}^{t}, t\right)= & w_{f} \frac{\psi(E) l}{m} q_{t_{v_{x}}} \log (10) \log \left(1+\frac{P_{v_{x}, f}^{t}\left|H_{v_{x}, v_{y}, f}\right|^{2}}{w_{f} N_{v_{y}, f}+I_{v_{y}, f}}\right)^{2}- \\
& w_{f} \log (2)\left(\alpha-2 \frac{\psi(E) l}{m} q_{t_{v_{x}}} C_{v_{y}, f}(t) \log (10)\right) \log \left(1+\frac{P_{v_{x}, f}^{t}\left|H_{v_{x}, v_{y}, f}\right|^{2}}{w_{f} N_{v_{y}, f}+I_{v_{y}, f}}\right)- \\
& \log (2)\left(w_{f} \alpha+C_{v_{y}, f}(t) \log (2)\left(\alpha-\frac{\psi(E) l}{m} q_{t_{v_{x}}} C_{v_{y}, f}(t) \log (10)\right)\right)
\end{aligned}
$$

To guarantee convergence to the global optimum, as all of the other parameters are real and positive, $\Delta\left(P_{v_{x}, f}^{t}, t\right)<0$ for strict convexity and is the necessary condition to ensure that $-L_{v_{x}, v_{y}, f}^{*^{\prime \prime}}\left(P_{v_{x}, f}^{t}, t\right)>0$. To confirm that $\Delta\left(P_{v_{x}, f}^{t}, t\right)<0$ for all three networks, we utilize the operating parameters from Tables 5.1 and 5.2, as well as typical values for $P_{v_{x}, f}^{t}, H_{v_{x}, v_{y}, f}, N_{v_{y}, f}$ and $I_{v_{y}, f}$, to summarize standard results for $\Delta\left(P_{v_{x}, f}^{t}, t\right)$ below,

Table C.1: Summary of $\Delta\left(P_{v_{x}, f}^{t}, t\right)$ for Zigbee, UWB and WiMax Networks

\begin{tabular}{ccccccc}
\hline Network & $\begin{array}{c}P_{v_{x}, f}^{t} \\
(\mathrm{dBm})\end{array}$ & $\begin{array}{c}\left|H_{v_{x}, v_{y}, f}\right|^{2} \\
(\mathrm{~dB})\end{array}$ & $\begin{array}{c}N_{v_{y}, f} \\
(\mathrm{~dB})\end{array}$ & $\begin{array}{c}I_{v_{y}, f} \\
(\mathrm{~dB})\end{array}$ & $\Delta\left(P_{v_{x}, f}^{t}, t\right)$ & $\Delta(0, t)$ \\
\hline Zigbee & -10 & -65 & -204.0 & -150 & $-2.1 \times 10^{9}$ & $-1.3 \times 10^{9}$ \\
UWB & -25 & -60 & -204.0 & -150 & $-1.9 \times 10^{13}$ & $-1.5 \times 10^{13}$ \\
WiMax & 10 & -80 & -204.0 & -150 & $-3.5 \times 10^{12}$ & $-2.9 \times 10^{12}$ \\
\hline
\end{tabular}

As $\Delta\left(P_{v_{x}, f}^{t}, t\right)<0$ for all three subnetworks, $-L_{v_{x}, v_{u}, f}^{*^{\prime \prime}}\left(P_{v_{x}, f}^{t}, t\right)>0$ in all cases including $P_{v_{x}, f}^{t}=0$. Given that a sum of convex functions is also convex, our objective function $-U_{v_{i}, v^{*}}^{a^{*}}$, as the summation of $-L_{v_{x}, v_{y}, f}^{*}$ along candidate paths in the sensor and mesh network, is also convex. Thus, our algorithm converges to the unique optimal resource allocation that leads to the maximum $U_{v^{\prime}, v_{\jmath}}^{a^{*}}$ given our objective function constraints. 